\title{
WestVirginiaUniversity
}

THE RESEARCH REPOSITORY @ WVU

Graduate Theses, Dissertations, and Problem Reports

2003

\section{Essays on the opportunity cost of constrained portfolio strategies}

Alla Anatolyevna Melkumian

West Virginia University

Follow this and additional works at: https://researchrepository.wvu.edu/etd

\section{Recommended Citation}

Melkumian, Alla Anatolyevna, "Essays on the opportunity cost of constrained portfolio strategies" (2003). Graduate Theses, Dissertations, and Problem Reports. 1831.

https://researchrepository.wvu.edu/etd/1831

This Dissertation is protected by copyright and/or related rights. It has been brought to you by the The Research Repository @ WVU with permission from the rights-holder(s). You are free to use this Dissertation in any way that is permitted by the copyright and related rights legislation that applies to your use. For other uses you must obtain permission from the rights-holder(s) directly, unless additional rights are indicated by a Creative Commons license in the record and/ or on the work itself. This Dissertation has been accepted for inclusion in WVU Graduate Theses, Dissertations, and Problem Reports collection by an authorized administrator of The Research Repository @ WVU.

For more information, please contact researchrepository@mail.wvu.edu. 


\title{
Essays on the Opportunity Cost of Constrained Portfolio Strategies
}

\author{
Alla A. Melkumian \\ Dissertation Submitted to the \\ College of Business and Economics \\ at West Virginia University \\ in partial fulfillment of the requirements \\ for the degree of \\ Doctor of Philosophy \\ in \\ Economics \\ Douglas W. Mitchell, Ph.D., Chair \\ Ronald J. Balvers, Ph.D. \\ Stratford M. Douglas, Ph.D. \\ Ashok B. Abbott, Ph.D. \\ Kern O. Kymn, Ph.D.
}

Division of Economics and Finance

Morgantown, West Virginia

2003

Keywords: Proportionate Opportunity Cost, Constrained Portfolio Strategy, Investors'

Welfare Losses

Copyright 2003 Alla A. Melkumian 


\title{
ABSTRACT \\ Essays on the Opportunity Cost of Constrained Portfolio Strategies
}

\begin{abstract}
Alla Melkumian
The dissertation explores the effect of different constrained portfolio strategies on investors' welfare. A constrained portfolio strategy gives investors a sub-optimal asset allocation that results in investors' welfare losses. To measure those welfare losses I compare constrained portfolios with optimal portfolios by using the concept of the proportionate opportunity cost along with various CRRA utility functions. Two sets of assets are used in calculations: the original historical asset returns data set, and the historical asset returns with extreme values exaggerated. In each data set case, with a nominally risk-free asset, a vector autoregression is used to generate the joint distribution of asset returns for the portfolio formation period, and 1,000 alternative sets of assets are randomly made available for investment.
\end{abstract}

Chapter 1 discusses reasons the investors use constrained portfolio strategies, describes the different constrained portfolio strategies that are used in the dissertation, derives the proportionate opportunity cost, describes the derivation of the joint probability distribution functions for asset returns, and describes the way the historical extreme asset returns values are exaggerated to check the robustness of the estimates of the proportionate opportunity cost.

The first essay, in Chapter 2, analyzes investors' welfare losses from being constrained to choose a mean-variance efficient portfolio. I show that the mean-variance strategy shows a moderately good approximation to the optimal portfolio strategy. When extreme values of returns are not exaggerated in the returns distribution, investors' welfare losses do not exceed $5.6 \%$ of initial wealth. With extreme returns values exaggerated in the returns distribution, investors' welfare losses do not exceed $11 \%$ of initial wealth. The results in both cases indicate that as the number of assets in investors' portfolios increases investors' welfare losses from the mean-variance constraint increase as well, and that less risk-averse investors experience greater welfare losses.

The second essay, in Chapter 3, analyzes investors' welfare losses from investing in a non-welldiversified number of assets. The analysis is performed with and without a short-selling constraint. I show that without the short-selling constraint and with no exaggeration of extreme values of asset returns the lowest well-diversified number of assets for an investor with low risk aversion is 24. The number decreases as the level of risk aversion increases, and when the shortselling constraint is introduced. When the extreme values of asset returns are exaggerated in the asset returns distribution the lowest well-diversified number of assets increases.

The second part of the chapter explores investors' welfare losses when they restrict themselves to either stocks or bonds but not both. I show that for investors with low levels of risk aversion welfare losses do not exceed $1.5 \%$ of initial wealth when they invest in only one type of assets. For investors with medium and high levels of relative risk aversion, constrained portfolios that include only one type of assets, stocks only or bonds only, along with Treasury bills, give expected utility about as high as unconstrained portfolios that include both types of assets, stocks and bonds. 
The third essay, in Chapter 4, analyzes investors' welfare losses from being restricted from short selling. I show that with a nominally risk-free asset the optimal portfolio strategy with the shortselling constraint performs almost as well as the unconstrained portfolio strategy for investors with medium levels of risk aversion, and performs as well as the unconstrained portfolio strategy for investors with high levels of risk aversion. The results, derived from the original historical asset returns data set with no exaggeration of extreme returns, show that investors' welfare losses reach $12.8 \%$ of initial wealth when risk aversion is low. With extreme returns exaggerated in the returns distribution, investors' welfare losses reach $13.5 \%$ of initial wealth. The results in both cases indicate that less risk-averse investors experience greater welfare losses, and that the shortselling constraint reduces the cost of sub-optimal diversification.

Chapter 5 provides the summary of the dissertation and discusses the directions for possible future research. 


\section{ACKNOWLEDGEMENTS}

Now that I have come to completing my degree, I know it would have been impossible to do it all on my own. Many people have helped me along the way.

I wish to express my sincere appreciation and gratitude to Douglas Mitchell without whom this body of work would not have been possible. Your role in my graduate school experience was much more than that of a dissertation advisor. Thank you for leaving your door always open, thank you for your inspiration and guidance, thank you for the encouraging comments and for all the caring that you have given to me.

I would also like to thank the members of my dissertation committee: Ronald Balvers, Stratford Douglas, Ashok Abbott and Kern Kymn, for all their comments and input on this work.

To Barbara Michniak, thank you for always being there if and when I needed you. To Duo Zhang and Christine Harrington, thank you for sharing valuable thoughts and information with me, thank you for all the comments and suggestions that you provided on this dissertation.

I am forever indebted to my parents, Anatoly and Svetlana Tolmachev, who taught me the value of knowledge. Their love and support was always with me, despite the great distance between us.

Finally, I would like to express my sincere thanks and appreciation to my husband, Arsen Melkumian, for all the support he has given me. His involvement in my work covered a wide spectrum from commenting on my research to helping me out with programming problems. 


\section{Table of Contents}

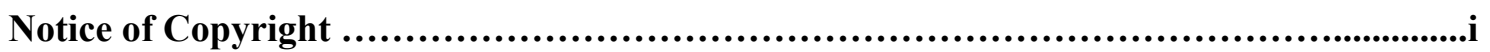

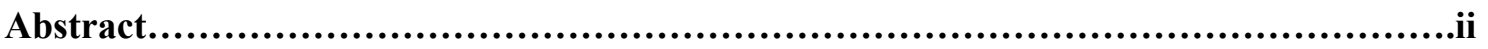

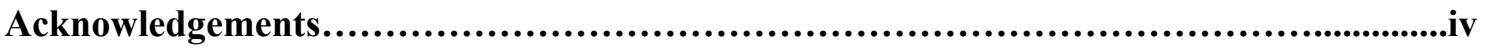

Table of Contents......................................................................v

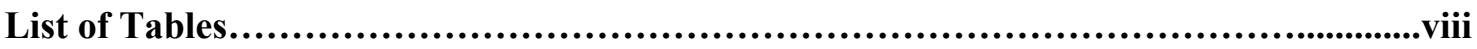

Chapter 1: Introduction 1

1. Constrained Portfolio Strategies.............................................

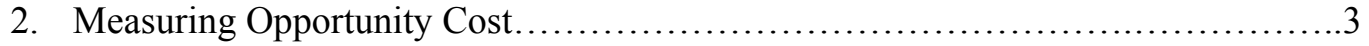

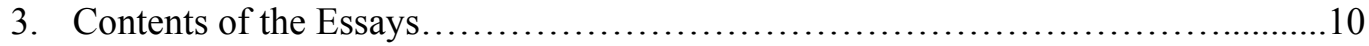

4. Inferring Joint Probability Distributions..................................... 12

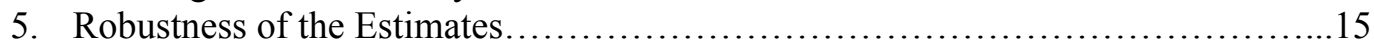

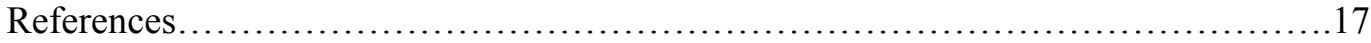

\section{Chapter 2: The Opportunity Cost for an Investor of Being Constrained}

by the Mean-Variance Framework $\quad 18$

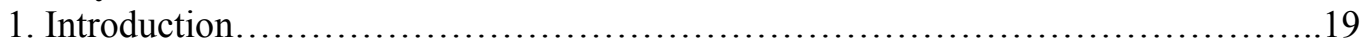

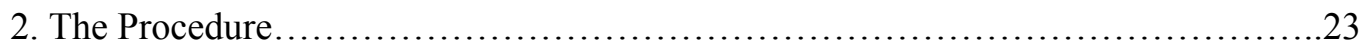

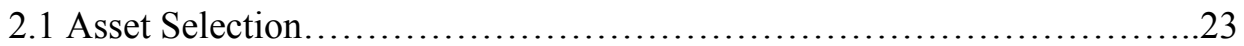

2.2.Vector Autoregressions of Returns....................................24

2.3 Mean-Variance Efficient Constrained Portfolios.........................26

2.4 Unconstrained Portfolios................................................28

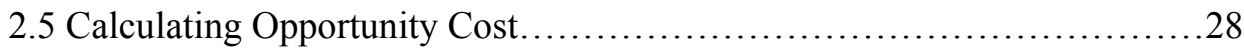

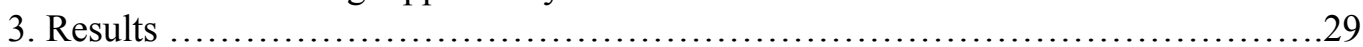

3.1 Results Derived from Historical Returns Data with no Exaggeration of Extreme Returns ...............................................29

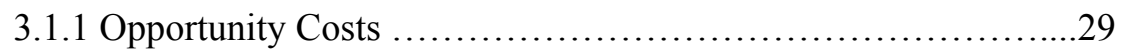

3.1.2 Optimal Portfolio Shares...................................35

3.1.3 Regret in the Worst-Case Scenario............................44

3.2 Historical Data with Extreme Values Exaggerated........................47

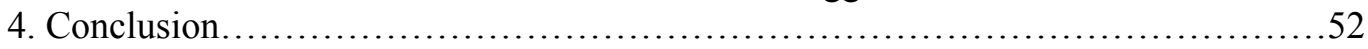

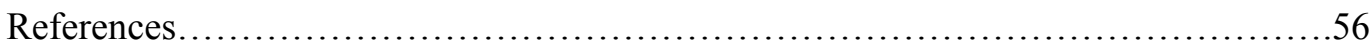

Chapter 3: The Opportunity Cost for an Investor of Being Constrained by the Number of Assets in His Portfolio $\quad 57$

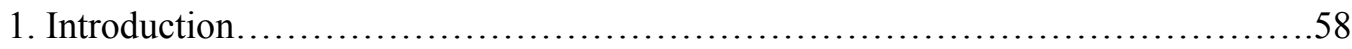

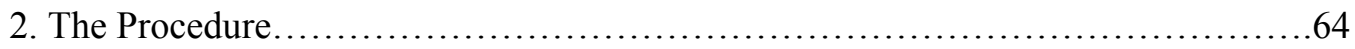

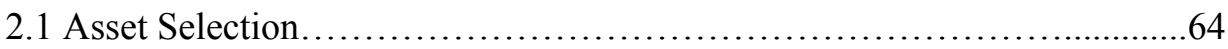

2.2.Vector Autoregressions of Returns.................................65

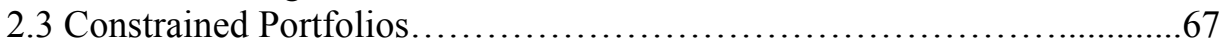

2.4 Unconstrained Portfolios.......................................67

2.5 Calculating Opportunity Cost.......................................68

2.6 Short-Selling Restriction.......................................... 70

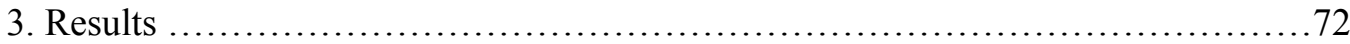

3.1 Results Derived for Opportunity Cost of Restricting the Number of Assets in Portfolios..................................................72 
3.1.1 Results Derived from Historical Returns Data with no Exaggeration of Extreme Returns

3.1.1.1 Opportunity Costs With and Without a

Short- Selling Constraint.

3.1.1.2 Optimal Portfolio Shares

3.1.2 Results Derived from Historical Returns Data with

Extreme Returns Exaggerated. .82

3.2 Results Derived for Opportunity Cost of Restricting the Number of Assets in Portfolios and of Using a Restricted VAR .86

3.2.1 Results Derived from Historical Returns Data with no Exaggeration of Extreme Returns .87

3.2.1.1 Opportunity Costs With and Without a Short-Selling Constraint

3.2.1.2 Optimal Portfolio Shares....

3.2.2 Results Derived from Historical Returns Data with

Extreme Returns Exaggerated..............................98

3.3 Summary of Results on Costs of Restricting the Number of Assets......102

4. The Opportunity Cost of Investing in Stocks only or Bonds only Versus

Investing in both Stocks and Bonds ........................................ 106

4.1 Vector Autoregressions of Returns..................................107

4.2 Constrained Portfolios.............................................107

4.3 Unconstrained Portfolios...........................................108

4.4 Calculating Opportunity Cost..........................................109

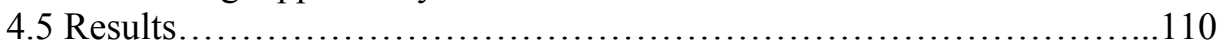

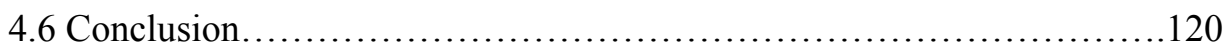

References................................................................... 122

Chapter 4: The Opportunity Cost for an Investor of Being Restricted from

$\begin{array}{lr}\text { Short-Selling } & 124\end{array}$

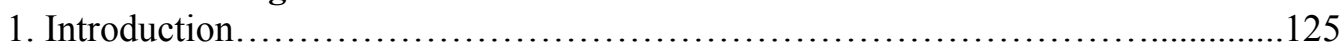

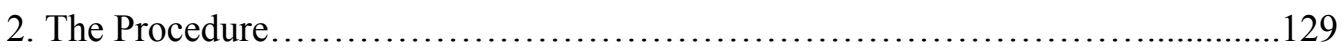

2.1 Asset Selection....................................................129

2.2.Vector Autoregressions of Returns...................................131

2.3 Constrained Portfolios............................................... 133

2.4 Unconstrained Portfolios..........................................134

2.5 Calculating Opportunity Cost.................................... 134

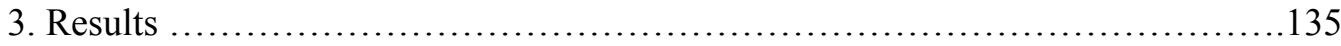

3.1 Results Derived from Historical Returns Data with No Exaggeration

of Extreme Returns ..............................................135

3.1.1 Opportunity Costs ........................................135

3.1.2 Optimal Portfolio Shares..................................140

3.1.3 Regret in the Worst-Case Scenario........................149

3.2 Historical Data with Extreme Values Exaggerated..........................152

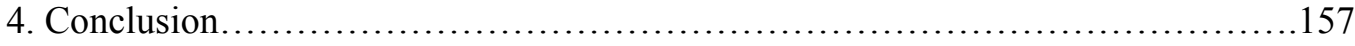

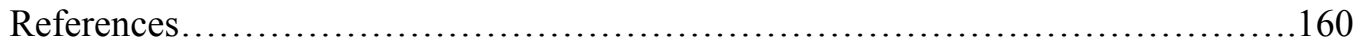

Chapter 5: Conclusions and Prospects for Future Research 161 


\section{APPENDICES:}

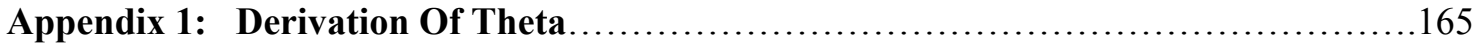

Appendix 2: A Sample Of SAS Program For Computing Opportunity Cost

Of The Mean-Variance Constraint .................................... 166 


\section{LIST OF TABLES}

\section{Chapter 2:}

Table 1. The Proportionate Opportunity Cost of the Mean-Variance Constraint, $(\theta-1)$, for Various Values of Relative Risk Aversion for 26 Assets

Table 2. The Proportionate Opportunity Cost of the Mean-Variance Constraint, $(\theta-1)$, for Various Values of Relative Risk Aversion for Nine Assets

Table 3. Illustrative Optimal Portfolio Shares for Unconstrained and Mean-Variance Constrained Portfolio Strategies for Different Values of Relative Risk Aversion for 26 Assets.

Table 4. Illustrative Optimal Portfolio Shares for Unconstrained and Mean-Variance Constrained Portfolio Strategies for Different Values of Relative Risk Aversion for Nine Assets.

Table 5. The Percentage of Certainty Equivalent Lost Due to the Mean-Variance Constraint for Various Levels of Relative Risk Aversion with No Exaggeration of Extreme Returns

Table 6. Illustrative Correlation Coefficients and Geometric Distances for Unconstrained and Mean-Variance Constrained Portfolios for Different Levels of Relative Risk Aversion for 26 Assets.

Table 7. Illustrative Correlation Coefficients and Geometric Distances for Unconstrained and Mean-Variance Constrained Portfolios for Different Levels of Relative Risk Aversion for Nine Assets.

Table 8. The Ex Post Proportionate Regret, $(\theta-1)$, Under the Worst Portfolio Outcome for 26 Assets for Unconstrained and Mean-Variance Constrained Portfolios .46

Table 9. The Ex Post Proportionate Regret, $(\theta-1)$, Under the Worst Portfolio Outcome for Nine Assets for Unconstrained and Mean-Variance Constrained Portfolios.

Table 10. The Proportionate Opportunity Cost of the Mean-Variance Constraint, $(\theta-1)$, for Various Values of Relative Risk Aversion with Extreme Returns Exaggerated for 26 Assets.

Table 11. The Proportionate Opportunity Cost of the Mean-Variance Constraint, $(\theta-1)$, for Various Values of Relative Risk Aversion with Extreme Returns Exaggerated for Nine Assets....

Table 12. The Percentage of Certainty Equivalent Lost Due to the Mean-Variance Constraint for Various Levels of Relative Risk Aversion with Extreme Returns Exaggerated. 


\section{Chapter 3:}

Table 1. The Proportionate Opportunity Cost of Restricting the Number of Assets in Portfolios, $(\theta-1)$, for Various Values of Relative Risk Aversion with no Short-Selling Constraint (mean values over 1,000 replications).

Table 2. The Proportionate Opportunity Cost of Restricting the Number of Assets in Portfolios, $(\theta-1)$, for Various Values of Relative Risk Aversion with the Short-Selling Constraint (mean values over 1,000 replications).

Table 3. Illustrative Optimal Portfolio Shares for Unconstrained and Optimally Constrained to Include $n$ Assets Portfolio Strategies for Different Values of Relative Risk Aversion (with no Short-Selling Constraint) 78

Table 4. Illustrative Optimal Portfolio Shares for Unconstrained and Optimally Constrained to Include $n$ Assets Portfolio Strategies for Different Values of Relative Risk Aversion with the Short-Selling Constraint

Table 5. The Proportionate Opportunity Cost of Restricting the Number of Assets in Portfolios, $(\theta-1)$, for Various Values of Relative Risk Aversion with Extreme Returns Exaggerated in the Asset Returns Distribution (with no Short-Selling Constraint; mean values over 1,000 replications)

Table 6. The Proportionate Opportunity Cost of Restricting the Number of Assets in Portfolios and of Using a Restricted VAR, $(\theta-1)$, for Various Values of Relative Risk Aversion with no Short-Selling Constraint (mean values over 1,000 replications).

Table 7. The Proportionate Opportunity Cost of Restricting the Number of Assets in Portfolios and of Using a Restricted VAR, $(\theta-1)$, for Various Values of Relative Risk Aversion with the Short-Selling Constraint (mean values over 1,000 replications).

Table 8. Illustrative Optimal Portfolio Shares for Unconstrained and Optimally Constrained to Include $n$ Assets and to Use a Restricted VAR Portfolio Strategies for Different Values of Relative Risk Aversion (with no Short-Selling Constraint)

Table 9. Illustrative Optimal Portfolio Shares for Unconstrained and Optimally Constrained to Include $n$ Assets and to Use a Restricted VAR Portfolio Strategies for Different Values of Relative Risk Aversion with the Short-Selling Constraint

Table 10. The Proportionate Opportunity Cost of Restricting the Number of Assets in Portfolios and of Using a Restricted VAR, $(\theta-1)$, for Various Values of Relative Risk Aversion with Extreme Returns Exaggerated in the Asset Returns Distribution (with no Short-Selling Constraint; mean values over 1,000 replications) 
Table 11. The Proportionate Opportunity Costs, $(\theta-1)$, Optimal Unconstrained Portfolio Weights and Optimal Ratios of Stock Indices to Bond Indices for Various Values of Relative Risk Aversion.

Table 12. Optimal Portfolio Shares for Unconstrained and Constrained to Include Stocks only or Bonds only Portfolios for Different Values of Relative Risk Aversion.

Table 13. The Percentage of Certainty Equivalent Lost Due to the "Only Stocks" and "Only Bonds" Constraints for Various Levels of Relative Risk Aversion

\section{Chapter 4:}

Table 1. The Proportionate Opportunity Cost of the Short-Selling Constraint, $(\theta-1)$, for Various Values of Relative Risk Aversion for the Well-Diversified Number of Assets.

Table 2. The Proportionate Opportunity Cost of the Short-Selling Constraint, $(\theta-1)$, for Various Values of Relative Risk Aversion for Ten Assets

Table 3. Illustrative Optimal Portfolio Shares for Unconstrained Portfolios and Portfolios with the Short-Selling Constraint for Different Values of Relative Risk Aversion for the Well-Diversified Number of Assets

Table 4. Illustrative Optimal Portfolio Shares for Unconstrained Portfolios and Portfolios with the Short-Selling Constraint for Different Values of Relative Risk Aversion for Ten Assets.

Table 5. The Percentage of Certainty Equivalent Lost Due to the Short-Selling Constraint for Various Levels of Relative Risk Aversion with No Exaggeration of Extreme Returns.

Table 6. Illustrative Correlation Coefficients and Geometric Distances for Unconstrained Portfolios and Portfolios with the Short-Selling Constraint for Different Levels of Relative Risk Aversion for the Well-Diversified Number of Assets.

Table 7. Illustrative Correlation Coefficients and Geometric Distances for Unconstrained Portfolios and Portfolios with the Short-Selling Constraint for Different Levels of Relative Risk Aversion for Ten Assets ....

Table 8. The Ex Post Proportionate Regret, $(\theta-1)$, Under the Worst Portfolio Outcome for the Well-Diversified Number of Assets.

Table 9. The Ex Post Proportionate Regret, $(\theta-1)$, Under the Worst Portfolio Outcome for Ten Assets. 
Table 10. The Proportionate Opportunity Cost of the Short-Selling Constraint, $(\theta-1)$, for Various Values of Relative Risk Aversion with Extreme Returns Exaggerated for the Well-Diversified Number of Assets. ....

Table 11. The Proportionate Opportunity Cost of the Short-Selling Constraint, $(\theta-1)$, for Various Values of Relative Risk Aversion with Extreme Returns Exaggerated for Ten Assets.

Table 12. The Percentage of Certainty Equivalent Lost due to the Short-Selling Constraint for Various Levels of Relative Risk Aversion with Extreme Returns Exaggerated .... 


\section{Chapter 1. Introduction}

\section{Constrained Portfolio Strategies}

The general maximization procedure for portfolio choice for an investor with multiple risky assets is

$$
\begin{gathered}
\operatorname{Max}_{\left\{\alpha_{1}, \ldots, \alpha_{n}\right\}} E U\left(w_{0} \alpha_{1} \widetilde{r}_{1}+\ldots+w_{0} \alpha_{n} \widetilde{r}_{n}\right) \\
\text { subject to } \alpha_{1}+\ldots+\alpha_{n}=1
\end{gathered}
$$

where $w_{0}$ is initial wealth, $\alpha_{i}$ is the portfolio share in asset $i, w_{0} \alpha_{i}$ is the number of dollars invested in asset $i, \widetilde{r}_{i}$ is the random return on asset $i, U\left(w_{0} \alpha_{1} \widetilde{r}_{1}+\ldots+w_{0} \alpha_{n} \widetilde{r}_{n}\right)$ is the investor's stochastic utility from investing in the portfolio of $n$ risky assets. There certainly is a cost of computing unconstrained portfolios in terms of programming effort and computer running time; therefore, some investors may consider constraining themselves to simpler portfolio strategies.

Apart from the simplification motive, there are other reasons for investors to use a constrained portfolio strategy rather than an unconstrained one. For example, the solution of (1) might require an investor to hold extremely long or extremely short investment positions. These extreme long or short holding positions sometimes are difficult to implement in practice because, investors face constraints on their portfolio holdings (e.g. Regulation $\mathrm{T}$ restricts almost all investors, individual as well as institutional, to $50 \%$ margins). Therefore, again, a constrained portfolio strategy may be considered.

Implementing a constrained portfolio strategy instead of an unconstrained one results in a welfare loss for the investor. How large can those welfare losses be? To answer the question I use historical data on asset returns to measure the opportunity cost of different types of constrained portfolio strategies in three essays: (i) the opportunity 
cost for the investor of being constrained by the mean-variance framework, (ii) the opportunity cost for the investor of being constrained by the number of assets in his portfolio and, in particular, the opportunity cost for the investor of having only bonds or only stocks in his portfolio instead of having both bonds and stocks, (iii) the opportunity cost for the investor of being restricted from short-selling.

(i) The mean-variance optimization as constrained portfolio strategy is used very frequently and has been the subject of many studies. The only problem with this optimization is that it is only optimal under certain assumptions about the distribution of asset returns or about the utility function of the investor: namely, that asset returns are elliptically distributed (or normally distributed, which is a specific case of elliptical distributions) or that the utility function is quadratic. These two assumptions are not plausible in real life: in real life statisticians frequently reject the hypothesis that asset returns are elliptically or even symmetrically distributed. And then there are absurd implications of a quadratic utility function of the investor (first, it is not globally increasing -- at some point it will decrease, and that will violate the presumption that first-order stochastically dominant improvements are never disliked; and second, it has increasing absolute risk aversion, which is not plausible). However, if the investor does choose to obtain the constrained optimal mean-variance efficient portfolio, all he needs is to minimize the variance of the portfolio given each particular level of expected wealth, and then choose the optimal one among the mean-variance-efficient portfolios that have been generated.

(ii) The next example of a constrained portfolio strategy is when an investor restricts himself to a certain number of assets in his portfolio and that number of assets is 
not a well-diversified one. That may happen even in efficient markets with all information available. Investors may depart significantly from the well-diversified allocation of funds by investing in too few assets. And this sub-optimal asset allocation will negatively affect their expected utility.

A particular case of this constrained portfolio strategy is portfolios that consist of stocks only or bonds only. Having invested in an "only bonds" or an "only stocks" portfolio the investor restricts himself to only one type of asset. It will create constrained portfolios because of the restriction. An unconstrained portfolio does not impose any restrictions on the asset type.

(iii) The next example of a constrained portfolio strategy is when an investor faces a restriction on short-selling. Short-selling is a legitimate trading strategy. By shortselling the investor will get extra money to invest and will make positive returns if the shorted assets price rises by less than that of the assets in which the extra funds are placed. At the same time it is a risky strategy because the investor will lose money if the price of the shorted asset rises by more than that of the assets in which the extra funds are placed. In any case restrictions on short-selling will reduce investment opportunities for the investor and will create a constrained investment strategy. Therefore, short-selling restrictions produce constrained portfolios.

\section{Measuring Opportunity Cost}

To measure the opportunity cost of different types of constrained portfolio strategies I use historical monthly asset returns for the period from January 1992 through December 2001. The ten most recent years of monthly asset returns give me the 120 most 
recent observations to work with, which balances recentness (relevance) of historical data versus the number of data points.

There is a chance, though, that with different time units I might get different conclusions. But changing monthly asset returns to quarterly or annual would bring additional problems: with quarterly asset returns I would need to extend the time period to 1972.I to 2001.IV and with annual asset returns it will be from 1882 to 2001 just to get the same 120 data points. In both cases I would have to deal with very old asset returns that might not accurately reflect the true probability distribution facing current investors. Therefore, my best choice of time units is monthly, which is what I use and which is also consistent with the previous studies of Simaan (1993) and Kroll, Levy and Markowitz (1984).

From a mathematical point of view all three essays in the dissertation use the same methodology. But from investors' point of view each essay deals with a separate problem in the portfolio theory: each essay describes and estimates welfare losses for investors from being engaged in a different constrained investment behavior.

A constrained portfolio strategy gives an investor a sub-optimal asset allocation. To calculate the investor's welfare loss from choosing or facing a constrained portfolio strategy, a standard technique is used. I cannot use just the difference between expected utilities because this calculation is not invariant to irrelevant addition or multiplication of a constant in the utility function.

The standard approaches (e.g. Brennan and Torous (1999)) to measure an individual's welfare cost are to use (a) willingness to accept payment as a fraction of initial wealth to accept the constraint, (b) willingness to pay of an investor as a fraction of 
initial wealth to stay with an unconstrained portfolio and avoid being constrained, or (c) the comparison of the certainty equivalents.

Under a constant relative risk aversion utility function (to be assumed) these three measures turn out to be equivalent: proportionate willingness to pay to avoid the constraint, $1.0-\varphi$, and proportionate willingness to accept the payment to accept the constraint, $\theta-1.0$, are related by $\psi=\frac{1}{\theta}$; and the ratio of the certainty equivalents of an unconstrained to a constrained optimal portfolio turns out to be equal to $\theta$.

(a) Dealing with willingness to accept payment means dealing with the proportionate opportunity cost, which gives the fraction of initial wealth that has to be accepted by an investor. Therefore, I need to define the proportionate opportunity cost as $\theta$ - 1.0. For example, the proportionate opportunity cost of $3 \%$ means that the investor being unconstrained will be equally happy as if he were constrained but had $3 \%$ more initial wealth.

The above use of the proportionate opportunity cost is natural because the results are readily interpretable as intuitively "large" or "small", which would not be true if compensating payments were expressed in additive dollar terms. In combination with the proportionate opportunity cost I use a constant relative risk aversion (CRRA) utility function. The decision to use this particular utility function is based on the fact that the function is commonly used in the literature on constrained portfolio strategies (e.g. Tew, Reid and Witt (1991), Brennan and Torous (1999)) and the fact that it is plausible (e.g. has decreasing absolute risk aversion (DARA) preferences) and mathematically tractable when combined with the proportionate opportunity cost. Hence, there are visible advantages in using both of them together. 
The CRRA utility function is:

$$
\mathrm{U}(\widetilde{w})=\left\{\begin{array}{l}
\frac{1}{\gamma} \widetilde{w}^{\gamma}, \gamma<1, \gamma \neq 0, \widetilde{w}>0 \\
-\infty, \widetilde{w} \leq 0
\end{array}\right.
$$

where $U(\widetilde{w})$ is the utility from final wealth, $\widetilde{w}$ is final wealth, and $l-\gamma$ is the level of relative risk aversion. Then, willingness to accept payment to accept the constraint, $\theta$ 1.0 , is defined by:

$$
\mathrm{E} \mathrm{U}\left(\theta w_{0} \widetilde{R}^{c}\right)=\mathrm{E} \mathrm{U}\left(w_{0} \widetilde{R}^{u}\right)
$$

where $w_{0}$ is initial wealth, $\widetilde{R}^{u}$ is the gross portfolio return (per dollar invested) from optimally investing without the constraint implied by the constrained portfolio strategy, $\widetilde{R}^{c}$ is the gross portfolio return from optimally investing subject to the constraint, $\mathrm{U}\left(\theta w_{0} \widetilde{R}^{c}\right)$ is utility from final wealth when the investor is being constrained and compensated, and $\mathrm{U}\left(w_{0} \widetilde{R}^{u}\right)$ is utility from final wealth when the investor is unconstrained and uncompensated.

Therefore, in the case of the CRRA utility (2) I have

$$
\mathrm{E}\left(\frac{1}{\gamma}\left(\theta w_{0} \widetilde{R}^{c}\right)^{\gamma}\right)=\mathrm{E}\left(\frac{1}{\gamma}\left(w_{0} \widetilde{R}^{u}\right)^{\gamma}\right)
$$

Starting at (4) I can solve for $\theta$, as shown in Appendix 1:

$$
\theta=\left[\frac{E\left(\widetilde{R}^{\gamma}\right)^{\text {unconstrained }}}{E\left(\widetilde{R}^{\gamma}\right)^{\text {constrained }}}\right]^{\frac{1}{\gamma}}
$$

Then $\theta-1.0$ tells me how much the investor will require to get paid as a fraction of initial wealth in order to accept the constrained portfolio. 
(b) But the fraction of initial wealth that the investor will be willing to pay to stay with the unconstrained portfolio, $1.0-\varphi$, is defined by:

$$
\mathrm{E} \mathrm{U}\left(w_{0} \widetilde{R}^{c}\right)=\mathrm{E} \mathrm{U}\left(\varphi w_{0} \widetilde{R}^{u}\right)
$$

where $\mathrm{U}\left(w_{0} \widetilde{R}^{c}\right)$ is the utility from the final wealth when the investor has the constrained portfolio, and $\mathrm{U}\left(\varphi w_{0} \widetilde{R}^{u}\right)$ is the utility from the final wealth when the investor pays the fraction $1.0-\varphi$ of initial wealth in order to avoid being constrained. Therefore, in case of the CRRA utility function, (2), I have:

$$
\mathrm{E}\left(\frac{1}{\gamma}\left(w_{0} \widetilde{R}^{c}\right)^{\gamma}\right)=\mathrm{E}\left(\frac{1}{\gamma}\left(\varphi w_{0} \widetilde{R}^{u}\right)^{\gamma}\right)
$$

Starting at (7) I can solve for $\varphi$ :

$$
\varphi=\left[\frac{E\left(\widetilde{R}^{\gamma}\right)^{\text {constrained }}}{E\left(\widetilde{R}^{\gamma}\right)^{\text {unconstrained }}}\right]^{\frac{1}{\gamma}}
$$

But (8) is the exact reciprocal of (5): $\varphi=\frac{1}{\theta}$. In other words, the proportion of initial wealth that the investor will have to be paid in order to stay with the constrained portfolio $(\theta-1.0)$ can be easily converted into the proportion of initial wealth that the investor will be willing to give up to stay with the unconstrained portfolio $(1.0-\varphi)$.

(c) The last appropriate measure of the proportionate opportunity cost is the ratio of the certainty equivalents. The certainty equivalent shows the amount of certain wealth that would be viewed with indifference by an investor relative to having an uncertain amount of wealth. I consider the ratio of the certainty equivalents of the unconstrained and constrained portfolios. 
Let me denote the certainty equivalent by CE; then, using the utility function (2) I get two expressions: the certainty equivalent for the investor with the unconstrained portfolio (9) and the certainty equivalent for the investor with the constrained portfolio (10):

$$
\begin{gathered}
\frac{1}{\gamma} \mathrm{CE}^{\gamma, \text { unconstrained }}=\frac{1}{\gamma} w_{0}^{\gamma} E\left(\widetilde{R}^{\gamma}\right)^{\text {unconstrained }} \\
\frac{1}{\gamma} \mathrm{CE}^{\gamma, \text { constrained }}=\frac{1}{\gamma} w_{0}^{\gamma} E\left(\widetilde{R}^{\gamma}\right)^{\text {constrained }}
\end{gathered}
$$

Starting from (9) and (10) I solve for the ratio of the certainty equivalents for the unconstrained and constrained portfolios:

$$
\frac{C E^{\text {unconstrained }}}{C E^{\text {constrained }}}=\left[\frac{E\left(\widetilde{R}^{\gamma}\right)^{\text {unconstrained }}}{E\left(\widetilde{R}^{\gamma}\right)^{\text {constrained }}}\right]^{\frac{1}{\gamma}}
$$

The right hand side of (11) is identical to the formula for $\theta$ (see (5)).

Under CRRA (1), one plus the proportionate willingness to accept payment and the ratio of the certainty equivalents of unconstrained to constrained optimal portfolios are equal. Since the ratio of the certainty equivalents is unitless and in particular has no time units, the proportionate opportunity cost, $\theta-1.0$, is timeless. But its value depends on a number of months until horizon. For example, with the investment horizon of one month if the unconstrained certainty equivalent is 1.08 and the constrained certainty equivalent is 1.03 , then the proportionate willingness to accept payment will be (1.08/1.03). But with a horizon of $T$ months the unconstrained certainty equivalent will be $1.08^{\mathrm{T}}$ and the constrained certainty equivalent will be $1.03^{\mathrm{T}}$. Then the ratio of the certainty equivalents is $(1.08 / 1.03)^{\mathrm{T}}$. Therefore, the proportionate willingness to accept payment, $\theta-1.0$, is a recurring cost. 
The three measures of the proportionate opportunity cost relative to wealth (willingness to accept payment, willingness to pay, and the ratio of the certainty equivalents) represent the right ways that can be used to compare constrained and unconstrained asset allocations and, therefore, the expected utilities from the constrained and unconstrained portfolios.

Even though a CRRA utility function is widely used, one can encounter a couple of problems in dealing with it.

The first problem comes from the fact, as Geweke (2001) pointed out, that if the probability distribution of a random variable is unbounded above (or below), then the expected utility, assuming a CRRA utility function of that random variable, could "equal" $+\infty($ or $-\infty)$. That makes the use of a CRRA in describing economic behavior limited. The problem, however, does not exist in my case, because all probability distributions that I work with are bounded above and below since they are the discrete probability distributions based on finite historical data.

Nevertheless, even if the probability distribution of a random variable is bounded above and below, as in my case, it still might be that the expected utility "equals" $-\infty$. In searching for the unconstrained optimal portfolio, it might happen that for a particular set of asset returns and for a particular set of portfolio shares one might end up with negative final wealth $(\widetilde{w}<0)$. In this case the utility from final wealth, using (2), will "equal" negative infinity. If a particular portfolio has a positive probability that such an outcome occurs, then the expected utility from final wealth will "equal" negative infinity too. This problem does not present any difficulty in my work at all: in searching for the unconstrained optimal portfolio I do the optimization procedure and through this 
optimization any set of portfolio shares that does not give the highest possible level of expected utility will not be picked. And as long as there exists at least one portfolio with $P(\widetilde{w} \leq 0)=0$, then a portfolio exists with $E U>-\infty$ and one such portfolio will be picked.

Therefore, the question of how large investors' welfare losses can be if they choose to use or face a constrained portfolio instead of an unconstrained one is answered by using the proportionate opportunity cost along with the CRRA utility function (2).

\section{Contents of the Essays}

In the first essay I calculate the proportionate opportunity cost for an investor who invests in the constrained optimal mean-variance efficient portfolio rather than in the unconstrained one. In the essay I show that as the level of relative risk aversion increases, the proportionate opportunity cost of choosing the mean-variance efficient optimal portfolio instead of unconstrained optimal portfolio decreases and the mean-variance strategy shows a moderately good approximation to the unconstrained portfolio strategy. The results, derived from the original historical asset returns data set with no exaggeration of extreme values of asset returns in the returns' distribution, show that investors' welfare losses do not exceed $5.6 \%$ of initial wealth. The results indicate that investors' welfare losses become larger when the number of assets in portfolios increases, and when extreme returns in the original historical data set exaggerated.

In the second essay I calculate the proportionate opportunity cost for an investor who invests in $n$ assets rather than in 26 assets, and I search for the smallest number that warrants being called well-diversified. In the essay I show that the well-diversified 
number of assets in one's portfolio depends on the degree of risk aversion, presence or absence of a short-selling constraint, and on the way a VAR process was used in deriving the asset returns distribution functions for the purpose of evaluating the proportionate opportunity cost. I find that when extreme values of asset returns are not exaggerated in the returns distribution, the smallest well-diversified number of assets in one's portfolio is 24 or less. When extreme values of asset returns are exaggerated, the smallest welldiversified number of assets is 25 or less. As relative risk aversion increases, the welldiversified number of assets in one's portfolio decreases. It is a counterintuitive conclusion but a clear reason emerges. The well-diversified number of assets also decreases when a short-selling constraint is introduced. The results also show that for investors with high levels of risk aversion the well-diversified number of assets is three or less due to the fact that they place about $90 \%$ of their initial wealth into Treasury bills.

In addition to that I look into a particular context for computing the welldiversified number of assets: the proportionate opportunity cost of investing in stocks only or in bonds only, but not in both stocks and bonds together. Through my calculations I find that as the level of relative risk aversion increases, the proportionate opportunity cost of investing in only one type of assets decreases. At the same time the highest proportionate opportunity cost I find is $1.6 \%$ of initial wealth that corresponds to "only bonds" portfolios. My findings of optimal ratios of stocks to bonds for different levels of risk aversion confirm the mutual-fund separation theorem's assertion that as the level of risk aversion increases, the optimal ratio of stocks to bonds stays constant. My results show that for investors with medium and high levels of relative risk aversion constrained portfolios that include only one type of assets, stocks only or bonds only 
along with Treasury bills, perform as well as the unconstrained portfolios that include both types of assets.

In the third essay I calculate the proportionate opportunity cost for an investor who faces a short-selling constraint on his portfolio holdings. In the essay I show that the portfolio strategy with the short-selling constraint performs almost as well as the unconstrained portfolio strategy for investors with medium levels of risk aversion, and performs as well as the unconstrained portfolio strategy for investors with high levels of risk aversion. The results, derived from the original historical asset returns data set with no exaggeration of extreme returns, show that investors' welfare losses reach $13.7 \%$ of initial wealth for investors with low levels of risk aversion; and become larger with extreme returns exaggerated in the return distribution.

\section{Inferring Joint Probability Distributions}

In each essay to calculate the proportionate opportunity cost I, first, pick at random a number of nominally risky assets; then I calculate their excess returns over the nominal risk-free return on Treasury bills. Next, using excess returns on those randomly picked assets I get the expected values of real returns for those risky assets and for the nominally risk-free Treasury bills at time $T+1$, the portfolio formation period, by using a Vector Autoregressive process (VAR). In real terms, though, there is no risk-free asset. Returns on Treasury bills are risk-free only in nominal terms. But in the time-series data the inflation rate will be uncertain in any period and, hence, so will the real rate of returns on Treasury bills. 
After getting the expected values of real returns at time $T+1$ I derive the joint probability distribution for the returns of the assets I have picked at random and Treasury bills. Finally, I construct the constrained and unconstrained optimal portfolios, and calculate the proportionate opportunity cost.

To derive the joint probability distribution of empirical deviations from the VARestimated conditional means for those randomly picked asset returns and inflation I do the following.

The nominal return on asset $i$ at time $t$ minus the nominal return on Treasury bills at time $t$ gives me the excess return on asset $i\left(x_{i, t}\right)$ at time $t$ for $i=1, \ldots, n$ and for $t=1, \ldots$, $T$. When I run a VAR for the excess returns of those randomly picked assets (say of three randomly picked assets, for example) and realized inflation as:

$$
\left[\begin{array}{l}
x_{1, t} \\
x_{2, t} \\
x_{3, t} \\
\pi_{t}
\end{array}\right]=\left[\begin{array}{l}
c_{1} \\
c_{2} \\
c_{3} \\
c_{4}
\end{array}\right]+\left[\begin{array}{llll}
v_{11}(L) & v_{12}(L) & v_{13}(L) & v_{14}(L) \\
v_{21}(L) & v_{22}(L) & v_{23}(L) & v_{24}(L) \\
v_{31}(L) & v_{32}(L) & v_{33}(L) & v_{34}(L) \\
v_{41}(L) & v_{42}(L) & v_{43}(L) & v_{44}(L)
\end{array}\right]\left[\begin{array}{c}
x_{1, t} \\
x_{2, t} \\
x_{3, t} \\
\pi_{t}
\end{array}\right]+\left[\begin{array}{c}
\varepsilon_{1, t} \\
\varepsilon_{2, t} \\
\varepsilon_{3, t} \\
\varepsilon_{\pi, t}
\end{array}\right]
$$

I obtain $\left\{\hat{c}_{i}\right\},\left\{\hat{\varepsilon}_{i, t}\right\}$ and $\left\{\hat{v}_{i, k}(L)\right\}$, where

$$
\hat{v}_{i, k}(L)=\hat{\delta}_{i, k}^{1} L^{1}+\hat{\delta}_{i, k}^{2} L^{2}+\ldots
$$

Then, I compute the vector of conditional expected values of excess returns for time $T+1$ and expected inflation for time $T+1$ as:

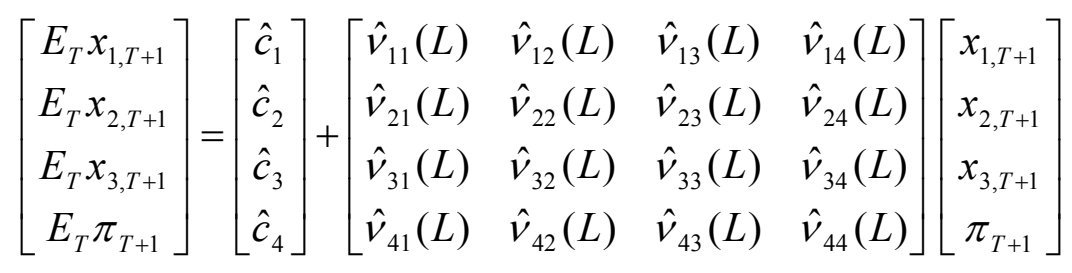

Next, the expected real return on asset $i$ in period $T+1$, the portfolio formation period, is 


$$
\left[\begin{array}{l}
E_{T} r_{1, T+1} \\
E_{T} r_{2, T+1} \\
E_{T} r_{3, T+1}
\end{array}\right]=\left[\begin{array}{l}
E_{T} x_{1, T+1} \\
E_{T} x_{2, T+1} \\
E_{T} x_{3, T+1}
\end{array}\right]+\left[\begin{array}{l}
r_{T B, T+1}^{n} \\
r_{T B, T+1}^{n} \\
r_{T B, T+1}^{n}
\end{array}\right]-\left[\begin{array}{l}
E_{T} \pi_{T+1} \\
E_{T} \pi_{T+1} \\
E_{T} \pi_{T+1}
\end{array}\right]
$$

where $r_{T B, T+1}^{n}$ is the ex ante observed nominal return on Treasury bills for time $T+1$. The expected real return on Treasury bills for time $T+1$ is

$$
E_{T} r_{T B, T+1}=r_{T B, T+1}^{n}-E_{T} \pi_{T+1}
$$

Finally, the conditional probability distribution for real returns for time $T+1$ is determined by

$$
\left[\begin{array}{c}
\widetilde{r}_{1, T+1} \\
\widetilde{r}_{2, T+1} \\
\widetilde{r}_{3, T+1} \\
\widetilde{r}_{T B, T+1}
\end{array}\right]=\left[\begin{array}{c}
E_{T} x_{1, T+1} \\
E_{T} x_{2, T+1} \\
E_{T} x_{3, T+1} \\
0
\end{array}\right]+\left[\begin{array}{c}
r_{T B, T+1}^{n} \\
r_{T B, T+1}^{n} \\
r_{T B, T+1}^{n} \\
r_{T B, T+1}^{n}
\end{array}\right]-\left[\begin{array}{c}
E_{T} \pi_{T+1} \\
E_{T} \pi_{T+1} \\
E_{T} \pi_{T+1} \\
E_{T} \pi_{T+1}
\end{array}\right]+\left[\begin{array}{c}
\widetilde{\varepsilon}_{1, T+1} \\
\widetilde{\varepsilon}_{2, T+1} \\
\widetilde{\varepsilon}_{3, T+1} \\
0
\end{array}\right]-\left[\begin{array}{c}
\widetilde{\varepsilon}_{\pi, T+1} \\
\widetilde{\varepsilon}_{\pi, T+1} \\
\widetilde{\mathcal{\varepsilon}}_{\pi, T+1} \\
\widetilde{\varepsilon}_{\pi, T+1}
\end{array}\right]
$$

where $\left[\begin{array}{c}\widetilde{\varepsilon}_{1, T+1} \\ \widetilde{\varepsilon}_{2, T+1} \\ \widetilde{\varepsilon}_{3, T+1} \\ \widetilde{\varepsilon}_{\pi, T+1}\end{array}\right]$ takes on the historically observed values $\left[\begin{array}{c}\widetilde{\varepsilon}_{1, t} \\ \widetilde{\varepsilon}_{2, t} \\ \widetilde{\varepsilon}_{3, t} \\ \widetilde{\varepsilon}_{\pi, t}\end{array}\right]$ from regression (12), $t=1,2, \ldots, T$, with equal probabilities $(1 / T)$.

The VAR procedure that I use to capture the dependence of the expected returns of nominally risky assets on their past histories, (12)-(14), was used in a similar context by Campbell and Viceira (2002) to calculate the expected values of returns on inflationindexed bonds. For the VAR procedure they assumed that the dynamics of all involved variables would be captured by a first-order vector autoregressive process, or VAR (1). The best way to approach the problem, which I follow here, is to determine the autoregressive order for the VAR by using the minimum AIC model selection procedure. 
AIC in this case is computed once for the entire group of four response equations in the VAR as opposed to the sum of four AIC computed for each response equation separately.

This way of deriving the asset returns probability distribution functions, using historically occurring innovations to asset returns captured through this VAR procedure, $(12)-(17)$, is superior to the VAR methods mentioned in the literature, e.g. Campbell and Viceira (2002). The literature on derivation of asset returns probability distribution functions assumes that the distribution of asset returns is static, not evolving over time. But the reality is such that the asset returns' distribution is dynamic, depending on both recent realizations and the fixed historical distribution of shocks to the dynamic asset returns' process. So, the right way of deriving asset returns probability distribution functions is to include the dynamics of the past history of asset returns.

\section{Robustness of the Estimates}

To check the robustness of the estimates of the proportionate opportunity cost I include extremely high and extremely low simulated asset returns in each data set of available assets.

I construct the simulated extremely high and extremely low asset returns the following way. For the original data set for each historical time period I compute the average excess return across all assets in the data set. A historical time period with the highest average excess return across all assets defines the historical period with the highest returns. A historical time period with the lowest average excess return across all assets defines the historical period with the lowest returns. Then, for the extreme historical periods only I calculate the deviation of each asset's return from that asset's 
intertemporal mean return. The deviations are doubled and then added back to assets' intertemporal means. This way I create two fictional time periods with exaggerated high and exaggerated low returns. These fictional asset returns provide simulated extreme time periods to replace the time periods they were constructed from. The rest of the original data set remains unchanged. This data set with fictional exaggerated high and exaggerated low asset returns is used to check the robustness of the estimates of the proportionate opportunity cost. 


\section{References:}

Brennan, M.J., W.N.Torous, Individual Decision-Making and Investor Welfare, Economic Notes, 1999, 28(2), 119-43

Campbell, John Y., Viceira, Luis H., Strategic Asset Allocation, Oxford University Press, March, 2002

Geweke, J., A note on some limitations of CRRA utility, Economics Letters, 2001, 71(3), $341-45$

Kroll, Yoram, Haim Levy and Harry M. Markowitz, Mean-Variance Versus Direct Utility Maximization, The Journal of Finance, 1984, 39(1), 47-61

Simaan, Yusif, What Is the Opportunity Cost of Mean-Variance Investment Strategy? Management Science, 1993, 39(5), 578-87 


\title{
Chapter 2. First Essay:
}

\section{The Opportunity Cost for an Investor of Being Constrained by the Mean-Variance Framework}

\begin{abstract}
Mean-variance analysis as a constrained portfolio strategy gives an investor a suboptimal asset allocation that results in a welfare loss for the investor. To measure that welfare loss I compare mean-variance constrained efficient portfolios with optimal unconstrained portfolios by using the concept of the proportionate opportunity cost along with various CRRA utility functions. Two sets of asset returns are used in calculations: the original historical asset returns data set, and the historical asset returns with extreme values exaggerated. In each case, with a nominally risk-free asset, a vector autoregression is used to generate the joint distribution of asset returns for the portfolio formation period, and 1,000 alternative sets of assets are randomly made available for investment. I show that for both types of data set the mean-variance strategy shows a moderately good approximation to the optimal portfolio strategy. When extreme values of returns are not exaggerated in the returns distribution, investors' welfare losses do not exceed $5.6 \%$ of initial wealth. With extreme returns values exaggerated in the returns distribution, investors' welfare losses do not exceed $11 \%$ of initial wealth. The results in both cases indicate that as the number of assets in investors' portfolios increases investors' welfare losses from the meanvariance constraint increase as well, and that less risk-averse investors experience greater welfare losses.
\end{abstract}




\section{Chapter 2. First Essay: \\ The Opportunity Cost for an Investor of Being Constrained by the \\ Mean-Variance Framework}

\section{Introduction}

Mean-variance analysis is used very frequently as a sub-optimal constrained portfolio strategy. Any constrained portfolio strategy will give an investor a sub-optimal asset allocation and that will result in the investor's experiencing welfare loss. How large can that welfare loss for the investor be if he has chosen the mean-variance-efficient portfolio instead of the optimal portfolio? In order to answer this question I will compare expected utility from the sub-optimal asset allocation (the mean-variance efficient constrained portfolio) with that from optimal asset allocation by using the concept of opportunity cost.

The introduction to this dissertation showed that proportionate opportunity cost is the best way to measure investors' welfare losses. Under the assumption of the constant relative risk aversion utility function

$$
\mathrm{U}(\widetilde{w})=\left\{\begin{array}{l}
\frac{1}{\gamma} \widetilde{w}^{\gamma}, \gamma<1, \gamma \neq 0, \widetilde{w}>0 \\
-\infty, \widetilde{w} \leq 0
\end{array}\right.
$$

the proportionate opportunity cost (willingness to accept payment as compensation for being constrained) can be calculated as $\theta-1.0$ where $\theta$ is defined by

$$
\operatorname{EU}\left(\theta w_{0} \widetilde{R}^{c}\right)=\operatorname{EU}\left(w_{0} \widetilde{R}^{u}\right)
$$

where $w_{0}$ is the initial wealth, and $\widetilde{R}^{u}$ and $\widetilde{R}^{c}$ are the stochastic returns per dollar invested for the unconstrained and constrained portfolios. Solving (2) gives 


$$
\theta=\left[\frac{E\left(\widetilde{R}^{\gamma}\right)^{\text {unconstrained }}}{E\left(\widetilde{R}^{\gamma}\right)^{\text {constrained }}}\right]^{\frac{1}{\gamma}} .
$$

Under CRRA, (1), one plus the proportionate willingness to accept payment and the ratio of certainty equivalents of unconstrained to constrained optimal portfolios are equal, as shown in the introduction of this dissertation. Since the ratio of certainty equivalents is unitless and in particular has no time units, the proportionate opportunity cost, $\theta-1.0$, is timeless. But its numerical value depends on a number of months until horizon, i.e. with the investment horizon of $T$ months the proportionate willingness to accept payment to accept the constraint is $\theta^{T}$.

The literature contains two examples of measuring the opportunity cost of using the mean-variance efficient portfolio instead of the optimal portfolio that I would like to discuss first.

Simaan (1993) used historical stock returns and their joint distribution was specified by a joint distribution, with a normal distribution of the idiosyncratic shocks conditional on a single common factor with the Pearson Type III class distribution. For a single period portfolio selection problem he derived closed-form solutions for the optimal portfolio under constant absolute risk aversion (CARA) utility function, and for the investor's second best choice, the best mean-variance efficient portfolio. Then he computed empirically the size of the optimization premium, $\rho$, for replacing the investor's second best choice by the optimal portfolio. This optimization premium, $\rho$, was expressed additively in dollar terms: “...[m]iminum amount on an invested dollar that an investor would require in order to replace his optimal strategy with his best meanvariance investment strategy". Thus Simaan's compensation $\rho$ is added to final wealth. In 
contrast, what I am going to do is to work with the proportionate opportunity cost, $\theta-1.0$ : that is, an optimization premium that is expressed as a fraction of initial wealth. If I were to construct in Simaan's context the analog of the proportionate opportunity cost, $\theta-1.0$, using his definition for the additive $\rho$, then the analog would equal $\frac{\rho}{w_{0}}$ and would depend on initial wealth (since his $\rho$ does not due to his use of CARA utility). The proportionate optimization premium expressed that way is not appealing because it can take on any value, large or small, depending on the level of initial wealth: for investors with lower initial wealth the proportionate optimization premium will be higher than for investors with high initial wealth. What we need is a way to express the proportionate optimization premium so it does not depend on initial wealth; the proportionate opportunity cost, $\theta-1.0$, along with CRRA, instead of CARA, is the way to do that.

Tew, Reid and Witt (1991) used a Monte Carlo approach to simulate asset returns distributions using various assumptions about random characteristics of hypothetical investments. Several parameterizations of CARA and CRRA utility functions were used, including some cases of extreme risk aversion characteristics. Simulated data sets of asset returns and these utility functions were used to compute the opportunity cost of accepting the mean-variance investment strategy. By using six different utility functions Tew, Reid and Witt wanted to illustrate the limits of the mean-variance approximation of optimal investment strategy. And that is why they chose extreme risk aversion characteristics. Looking for the limits of the mean-variance investment strategy the authors employed the concept of additive opportunity cost in dollar terms used by Simaan. And as I have mentioned before, the concept is not appealing. The best way to approach the problem of computing the opportunity cost, which I will follow here, is to use the proportionate 
opportunity cost along with the CRRA utility function (1). I will use different levels of risk aversion to illustrate the limits of the mean-variance investment strategy.

My way of deriving joint probability distributions of asset returns is different from that of Simaan and Tew, Reid and Witt. Simaan assumed a particular class of joint distributions fitted to historical data, and Tew, Reid and Witt simulated data from assumed security returns distributions. In both cases optimized portfolios were based on those assumed distributions. I am going to use a vector autoregressive process (VAR) to project the means of returns and to capture 120 historically occurring shocks to all asset returns, and then I will assume that the true distribution of shocks for the investment period is given by those 120 sets of returns shocks with equal probabilities. I do not fit a continuous distribution to the data because the results may be sensitive to the assumed distribution. The present way of deriving joint probability distributions provides the most defensible representation of the current asset returns distributions facing investors in the portfolio formation period.

The procedure of calculating the proportionate opportunity cost for an investor of being constrained by the mean-variance framework includes random asset selection for investors' portfolios, estimation of a vector autoregressive process, derivation of the joint probability distribution function of asset returns, and computing mean-variance efficient constrained optimal and unconstrained portfolios.

In this essay I show that with a nominally risk-free asset, as relative risk aversion increases the mean-variance strategy shows a moderately good approximation to the optimal portfolio strategy. The results, derived from the original historical asset returns data set, show that investors' welfare losses do not exceed $5.6 \%$ of initial wealth. The 
results also show investors' welfare losses become larger when the number of assets in portfolios increases, and when extreme returns in the original historical data set exaggerated.

The second section of this essay describes the procedure of random asset selection for investors' portfolios, of inferring the joint probability distribution function of asset returns, of computing the mean-variance efficient constrained optimal and unconstrained optimal portfolios, and the calculation of the proportionate opportunity cost. The third section discusses the results of the study, and the fourth section concludes.

\section{The Procedure}

\subsection{Asset Selection}

The procedure of calculating the proportionate opportunity cost for different levels of risk aversion will be performed 1,000 times, in each case using 25 randomly picked nominally risky assets and Treasury bills as the nominally risk-free asset. Then, the entire procedure will be repeated for eight randomly picked nominally risky assets and Treasury bills.

The first step is to pick at random 25 nominally risky assets. Then, to construct the optimal constrained and optimal unconstrained portfolios I need to get expected values of real returns for the 26 assets I am using for time $T+1$ : for the 25 nominally risky assets and for nominally risk-free Treasury bills. In real terms, though, there is no risk-free asset. Returns on Treasury bills are risk-free only in nominal terms. But in timeseries data inflation will be uncertain in any period and, thus, so will the real rate of 
return on Treasury bills. Therefore, the 26 assets that I am dealing with in real terms will all be risky assets. The same procedures are also conducted with nine assets instead of 26 .

A set of nine or 26 assets is big enough to give reliable estimates of optimal constrained and optimal unconstrained portfolios and, therefore, for the opportunity cost. Simaan (1993) argued that 10 stocks would be sufficient to trace the efficient frontier and Tew, Reid and Witt (1991) used from two to nine stocks in their calculations.

\subsection{Vector Autoregressions of Returns}

So, to get expected values of real returns for the case of 26 assets at time $T+1$, the portfolio formation period, I estimate a vector autoregressive process (VAR). The next steps are to derive the joint probability distribution for the 26 assets' real returns, and, finally, to construct optimal constrained and optimal unconstrained portfolios.

To derive the joint probability distribution of empirical deviations from the VARestimated conditional means for those randomly picked asset returns I do the following.

The nominal return on asset $i$ at time $t$ minus the nominal return on Treasury bills at time $t$ gives us the excess return on asset $i\left(x_{i, t}\right)$ at time $t$ for $i=1, \ldots, 25$ and for $t=1, \ldots, T$. When I run VAR for excess returns of those 25 assets and realized inflation, as

$$
\left[\begin{array}{c}
x_{1, t} \\
\cdot \\
x_{25, t} \\
\pi_{t}
\end{array}\right]=\left[\begin{array}{c}
c_{1} \\
\cdot \\
c_{25} \\
c_{26}
\end{array}\right]+\left[\begin{array}{cccc}
v_{1,1}(L) & \cdot & \cdot & v_{1,26}(L) \\
\cdot & \cdot & \cdot & \cdot \\
v_{25,1}(L) & \cdot & \cdot & v_{25,26}(L) \\
v_{26,1}(L) & \cdot & \cdot & v_{26,26}(L)
\end{array}\right]\left[\begin{array}{c}
x_{1, t} \\
\cdot \\
x_{25, t} \\
\pi_{t}
\end{array}\right]+\left[\begin{array}{c}
\varepsilon_{1, t} \\
\cdot \\
\varepsilon_{25, t} \\
\varepsilon_{\pi, t}
\end{array}\right],
$$

I obtain $\left\{\hat{c}_{i}\right\},\left\{\hat{\varepsilon}_{i, t}\right\}$ and $\left\{\hat{v}_{i, k}(L)\right\}$, where

$$
\hat{v}_{i, k}(L)=\hat{\delta}_{i, k}^{1} L^{1}+\hat{\delta}_{i, k}^{2} L^{2}+\ldots
$$


Then, I compute the vector of conditional expected values of excess returns for time $T+1$ and expected inflation for time $T+1$ as:

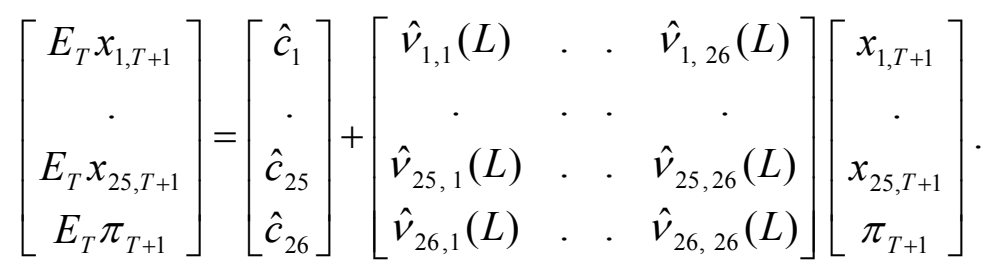

Then, the expected real return on asset $i$ in period $T+1$, the portfolio formation period, is

$$
\left[\begin{array}{c}
E_{T} r_{1, T+1} \\
\cdot \\
E_{T} r_{25, T+1}
\end{array}\right]=\left[\begin{array}{c}
E_{T} x_{1, T+1} \\
\cdot \\
E_{T} x_{25, T+1}
\end{array}\right]+\left[\begin{array}{c}
r_{T B, T+1}^{n} \\
\cdot \\
r_{T B, T+1}^{n}
\end{array}\right]-\left[\begin{array}{c}
E_{T} \pi_{T+1} \\
\cdot \\
E_{T} \pi_{T+1}
\end{array}\right]
$$

where $r_{T B, T+1}^{n}$ is the ex ante observed nominal return on Treasury bills for time $T+1$. The expected real return on Treasury bills for time $T+1$ is

$$
E_{T} r_{T B, T+1}=r_{T B, T+1}^{n}-E_{T} \pi_{T+1} .
$$

Then, the conditional probability distribution for real returns for time $\mathrm{T}+1$ is determined by

$$
\left[\begin{array}{c}
\widetilde{r}_{1, T+1} \\
\cdot \\
\widetilde{r}_{25, T+1} \\
\widetilde{r}_{T B, T+1}
\end{array}\right]=\left[\begin{array}{c}
E_{T} x_{1, T+1} \\
\cdot \\
E_{T} x_{25, T+1} \\
0
\end{array}\right]+\left[\begin{array}{c}
r_{T B, T+1}^{n} \\
\cdot \\
r_{T B, T+1}^{n} \\
r_{T B, T+1}^{n}
\end{array}\right]-\left[\begin{array}{c}
E_{T} \pi_{T+1} \\
\cdot \\
E_{T} \pi_{T+1} \\
E_{T} \pi_{T+1}
\end{array}\right]+\left[\begin{array}{c}
\widetilde{\varepsilon}_{1, T+1} \\
\cdot \\
\widetilde{\varepsilon}_{25, T+1} \\
0
\end{array}\right]-\left[\begin{array}{c}
\widetilde{\varepsilon}_{\pi, T+1} \\
\cdot \\
\widetilde{\varepsilon}_{\pi, T+1} \\
\widetilde{\varepsilon}_{\pi, T+1}
\end{array}\right]
$$

where $\left[\begin{array}{c}\widetilde{\varepsilon}_{1, T+1} \\ \cdot \\ \widetilde{\varepsilon}_{25, T+1} \\ \widetilde{\varepsilon}_{\pi, T+1}\end{array}\right]$ takes on the historically observed values $\left[\begin{array}{c}\widetilde{\varepsilon}_{1, t} \\ \cdot \\ \widetilde{\varepsilon}_{25, t} \\ \widetilde{\varepsilon}_{\pi, t}\end{array}\right], t=1,2, \ldots, T$, with equal

probabilities $(1 / T)$.

This way of deriving asset returns probability distribution functions, using historically occurring innovations to asset returns captured through this VAR procedure, 
is superior to the VAR method mentioned in the literature, e.g. Campbell and Viceira (2002). The literature on derivation of asset returns probability distribution functions assumes that the history of asset returns is static, with the ex ante distribution of returns not evolving over time. But the reality is such that the history of asset returns is dynamic. Ex ante asset returns' distributions depend on both recent realizations and the fixed historical distribution of shocks to the dynamic asset returns' process. So the right way of deriving asset returns probability distribution functions is to include the dynamics of the past history of asset returns.

\subsection{Mean-Variance Efficient Constrained Portfolios}

Using the information about those randomly picked assets' derived probability distributions for their real returns (computed as shown in (9)), I compute two meanvariance efficient mutual funds, $X_{1}^{*}$ and $X_{2}^{*}$, using the following formula (Merton (1972)):

(10) $X_{i}^{*}=\frac{w_{0}}{\Delta}\left[\left(\bar{r}^{\prime} V^{-1} \bar{r}\right) V^{-1} k-\left(k^{\prime} V^{-1} \bar{r}\right) V^{-1} \bar{r}\right]+\frac{\mu_{i}}{\Delta}\left[\left(k^{\prime} V^{-1} k\right) V^{-1} \bar{r}-\left(\bar{r}^{\prime} V^{-1} k\right) V^{-1} k\right], i=1,2$

where

$$
\Delta=\left(\bar{r}^{\prime} V^{-1} \bar{r}\right)\left(k^{\prime} V^{-1} k\right)-\left(\bar{r}^{\prime} V^{-1} k\right)^{2}>0 .
$$

Here $w_{0}$ is the initial wealth that is set equal to $l, \bar{r}$ is a column vector with dimension of $26 x 1$ of expected values of the gross real returns, $\widetilde{r}_{t}$, on 25 nominally risky assets for time $T+1$ and expected real return on Treasury bills for time $T+1$ calculated as shown in (7) and (8), $X_{i}^{*}$ is a mean-variance efficient mutual fund (a column vector of portfolio shares for the 25 picked assets and Treasury bills) with dimension $26 x 1, \mu_{i}$ is expected 
gross portfolio return (for two different mean-variance efficient mutual funds I pick two different arbitrary values for $\mu$ ), $V$ is the covariance matrix of real returns with dimension of $26 \times 26$ for the 26 risky assets calculated from the distribution of $\left[\begin{array}{c}\widetilde{\varepsilon}_{1, T+1} \\ \cdot \\ \widetilde{\varepsilon}_{25, T+1} \\ 0\end{array}\right]-\left[\begin{array}{c}\widetilde{\varepsilon}_{\pi, T+1} \\ \cdot \\ \widetilde{\varepsilon}_{\pi, T+1} \\ \widetilde{\varepsilon}_{\pi, T+1}\end{array}\right]$ stated after (9), and $k$ is the column vector of 1 's with dimension $26 x 1$.

Computing two mean-variance efficient mutual funds gives us two column vectors, $X_{1}^{*}$ and $X_{2}^{*}$, of portfolio shares for the 26 assets for the two mean return levels, $\mu_{1}$ and $\mu_{2}$.

The next step is to optimize the expected value of a CRRA utility function, (1), subject to the constraint of being mean-variance efficient, with respect to the single choice variable $\beta$ : how much to hold in one of the efficient mutual funds as opposed to the other:

$$
\underset{\{\beta\}}{\operatorname{Max}} \operatorname{EU}(\widetilde{w})=\mathrm{E}\left\{\frac{1}{\gamma} \widetilde{w}^{\gamma}\right\} \text { subject to } \widetilde{w}=\left[\beta\left(X_{1}^{*^{\prime}} \widetilde{r}_{t}\right)+(1-\beta)\left(X_{2}^{*} \widetilde{r}_{t}\right)\right] w_{0}
$$

where $w_{0}$ is the initial wealth that is set equal to 1 , the expectation is taken over the joint probability distribution derived as described above in (4)-(9), and the time subscripts on $E_{T}$ and $\widetilde{w}_{T+1}$ have been suppressed for convenience.

By solving the above problem (finding the hilltop in the graph with the Expected utility on the vertical axis and $\beta$ (portfolio share of mutual fund number one) on the horizontal axis) I get the mean-variance efficient constrained optimal portfolio. 


\subsection{Unconstrained Portfolios}

The next step, then, is to get the unconstrained optimal portfolio: the solution of

$$
\underset{\left\{\alpha_{1}, \ldots, \alpha_{25}\right\}}{\operatorname{Max}} E U(\widetilde{w})=\operatorname{Max} E\left\{\frac{1}{\gamma}\left[w_{0}\left(\alpha_{1} \widetilde{r}_{1}+\ldots+\alpha_{25} \widetilde{r}_{25}+\left(1-\alpha_{1}-\ldots-\alpha_{25}\right) \widetilde{r}_{T B}\right)\right]^{\gamma}\right\}
$$

where $\alpha_{1}, \ldots, \alpha_{25}$ are the first 25 individual assets portfolio shares in the unconstrained optimal portfolio. To get the portfolio I search over $\alpha_{1}, \ldots, \alpha_{25}$ space to optimize expected utility, using nonlinear optimization by a quasi-Newton method based on convergence to first-order conditions of problem (13). Again, the expectation is taken over the joint probability distribution derived as described above in (4)-(9).

\subsection{Calculating Opportunity Cost}

Now, when I have the constrained optimal and unconstrained optimal portfolios I calculate the opportunity cost, $\theta-1.0$. For the formula for $\theta$, (3), I need to find $\mathrm{E}\left(\widetilde{R}^{\gamma}\right)^{\text {unconstrained }}$ and $\mathrm{E}\left(\widetilde{R}^{\gamma}\right)^{\text {constrained }}$.

$\mathrm{E}\left(\widetilde{R}^{\gamma}\right)^{\text {unconstrained }}$ (referring more completely to $E_{T}\left(\widetilde{R}_{T+1}^{\gamma}\right)^{\text {unconstrained }}$ ) is equal to

$$
E_{T}\left(\widetilde{R}_{T+1}^{\gamma}\right)^{\text {unconstrd }}=\frac{1}{T} \sum_{t=1}^{T}\left\{\left[\begin{array}{llll}
\alpha_{1}^{*} & \ldots & \alpha_{25}^{*} & 1-\alpha_{1}^{*}-\ldots-\alpha_{25}^{*}
\end{array}\right]\left[\begin{array}{c}
E_{T} r_{1, T+1}+\varepsilon_{1, t}-\varepsilon_{\pi, t} \\
\cdot \\
E_{T} r_{25, T+1}+\varepsilon_{25, t}-\varepsilon_{\pi, t} \\
E_{T} r_{T B, T+1}+0-\varepsilon_{\pi, t}
\end{array}\right]\right\}^{\gamma}
$$

where the vector of $\alpha_{i}^{*}$ is the vector of optimal portfolio shares; the vector of $E_{T} r_{i, T+1}+\varepsilon_{i, t}-\varepsilon_{\pi, t}$ and $E_{T} r_{T B, T+1}-\varepsilon_{\pi, \mathrm{t}}$ is the vector of particular possible values of real returns (conditional on data set for times $t=1$ through $T$ ) at time $T+1$ (the portfolio formation period) and calculated as shown in (4)-(9). 
And $\mathrm{E}\left(\widetilde{R}^{\gamma}\right)^{\text {constrained }}$ is equal to

$$
E_{T}\left(\widetilde{R}_{T+1}^{\gamma}\right)^{\text {constrained }}=\frac{1}{T} \sum_{t=1}^{T}\left\{\left[\beta^{*} X_{1}^{*}+\left(1-\beta^{*}\right) X_{2}^{*}\right]\left[\begin{array}{c}
E_{T} r_{1, T+1}+\varepsilon_{1, t}-\varepsilon_{\pi, t} \\
\cdot \\
E_{T} r_{25, T+1}+\varepsilon_{25, t}-\varepsilon_{\pi, t} \\
E_{T} r_{T B, T+1}+0-\varepsilon_{\pi, t}
\end{array}\right]\right\}^{\gamma}
$$

where $\beta^{*}$ is the portfolio share of mutual fund number one and $\left(1-\beta^{*}\right)$ is the portfolio share of mutual fund number two; $X_{i}^{*}$, for $i=1,2$, are two mean-variance efficient mutual funds.

Then, having calculated (14) and (15), I use (3) to get a numerical value for $\theta$.

The whole procedure, starting from picking 25 (or eight) nominally risky assets, is being repeated 1,000 times. This gives me 1,000 values of $\theta$. The procedure is done for each of 11 alternative values of the risk aversion parameter $\gamma$.

\section{Results}

The results from this research project are as follows.

\subsection{Results Derived from Historical Returns Data with no Exaggeration of Extreme \\ Returns.}

\subsubsection{Opportunity Costs}

Table 1 and Table 2 represent the results from calculation of 1,000 values of the proportionate opportunity cost for 11 different values of relative risk aversion for alternatively 26 and nine assets, based on historically occurring asset returns over the 10year period January 1992 through December 2001. 
Table 1

The proportionate opportunity cost of the mean-variance constraint, $(\theta-1)$, for various values of relative risk aversion for 26 assets

\begin{tabular}{|c|c|c|c|c|c|}
\hline $\begin{array}{c}\text { Relative } \\
\text { Risk Aversion, (1- } \gamma)\end{array}$ & Smallest & Mean & Median & Largest & Standard Deviation \\
\hline \multicolumn{6}{|l|}{ Low } \\
\hline 0.7 & 0.008 & 0.056 & 0.044 & 0.334 & 0.030 \\
\hline 1 & 0.006 & 0.048 & 0.027 & 0.182 & 0.032 \\
\hline 2 & 0.002 & 0.035 & 0.016 & 0.125 & 0.029 \\
\hline 3 & 0.001 & 0.032 & 0.014 & 0.114 & 0.022 \\
\hline \multicolumn{6}{|l|}{ Medium } \\
\hline 9 & 0.000 & 0.030 & 0.001 & 0.111 & 0.019 \\
\hline 10 & 0.000 & 0.028 & 0.001 & 0.088 & 0.019 \\
\hline 11 & 0.000 & 0.024 & 0.001 & 0.075 & 0.018 \\
\hline 12 & 0.000 & 0.019 & 0.001 & 0.040 & 0.016 \\
\hline \multicolumn{6}{|l|}{ High } \\
\hline 29 & 0.000 & 0.012 & 0.001 & 0.042 & 0.012 \\
\hline 30 & 0.000 & 0.006 & 0.000 & 0.041 & 0.012 \\
\hline 31 & 0.000 & 0.005 & 0.000 & 0.028 & 0.011 \\
\hline
\end{tabular}

Table 2

The proportionate opportunity cost of the mean-variance constraint, $(\theta-1)$, for various values of relative risk aversion for nine assets

\begin{tabular}{|c|c|c|c|c|c|}
\hline $\begin{array}{c}\text { Relative } \\
\text { Risk Aversion, }(1-\gamma)\end{array}$ & Smallest & Mean & Median & Largest & Standard Deviation \\
\hline \multicolumn{6}{|l|}{ Low } \\
\hline 0.7 & 0.001 & 0.035 & 0.004 & 0.593 & 0.104 \\
\hline 1 & 0.000 & 0.028 & 0.002 & 0.580 & 0.058 \\
\hline 2 & 0.000 & 0.014 & 0.001 & 0.571 & 0.028 \\
\hline $\begin{array}{c}3 \\
\text { Medium }\end{array}$ & 0.000 & 0.011 & 0.001 & 0.395 & 0.020 \\
\hline 9 & 0.000 & 0.009 & 0.006 & 0.321 & 0.018 \\
\hline 10 & 0.000 & 0.007 & 0.006 & 0.189 & 0.019 \\
\hline 11 & 0.000 & 0.007 & 0.005 & 0.093 & 0.018 \\
\hline 12 & 0.000 & 0.006 & 0.005 & 0.080 & 0.018 \\
\hline \multicolumn{6}{|l|}{ High } \\
\hline 29 & 0.000 & 0.005 & 0.005 & 0.061 & 0.017 \\
\hline 30 & 0.000 & 0.004 & 0.004 & 0.055 & 0.017 \\
\hline 31 & 0.000 & 0.004 & 0.003 & 0.012 & 0.014 \\
\hline
\end{tabular}


Of all the values of relative risk aversion examined the lowest mean (over 1,000 replications) of the proportionate opportunity cost for both 26 and nine assets corresponds to the high level of relative risk aversion of 31 . The highest mean (over 1,000 replications) of the proportionate opportunity cost for both 26 and nine assets corresponds to the lowest level of relative risk aversion of 0.7 . This suggests that optimal unconstrained portfolios offer high risk-tolerance investors broader, more daring investment opportunities than constrained optimal portfolios, and so the investors will require a premium to give up those investment opportunities.

Both tables clearly show that as level of relative risk aversion increases the proportionate opportunity cost decreases, given the CRRA utility function, (1), the better the mean-variance efficient portfolio performs and the lower the proportion of initial wealth an investor requires to stay constrained and accept the mean-variance efficient portfolio instead of optimal unconstrained portfolio. This is not surprising. As risk aversion decreases, as investor becomes more risk tolerant, he considers optimal unconstrained portfolio as his best choice that does not place any restrictions on his investment behavior and let him follow a very aggressive short sale strategy that will not be possible under the constrained portfolio strategy (see Table 3 and Table 4), and, therefore, he will require higher proportion of initial wealth as the payment to stay constrained and accept the optimal constrained mean-variance efficient portfolio.

These results confirm Simaan's (1993) conclusions in the case where a riskless asset was introduced. He also found that as the level of risk aversion increases the optimization premium will decrease. What differs, though, between his results and mine is the magnitude of the estimates of the proportional opportunity cost. 
The highest opportunity cost that Simaan found is $30 \%$ for the case with no riskless asset and $0.5 \%$ for the case with the riskless asset. The highest opportunity cost I have found is $5.6 \%$ for 26 assets and $3.5 \%$ for nine assets (in both my cases a nominally risk-free asset, risky in real terms, was introduced). The difference can be explained, first, by the use of the utility function with constant relative risk aversion preferences rather than utility function with constant absolute risk aversion preferences as in Simaan, and second, by the fact that my highest proportionate opportunity cost corresponds to the level of risk aversion of 0.7 whereas Simaan's highest opportunity cost corresponds to the level of risk aversion of 2 (he deliberately did not include levels of risk aversion less then 2 arguing that they correspond to very aggressive infeasible investment strategies).

Tew, Reid and Witt (1991), working only with risky assets and relative risk aversion that ranged from 0.1 to 1.9 (therefore, with various CRRA utility functions), found that as risk aversion increases the opportunity cost increases too. Their findings are consistent with Simaan's case where there was no riskless asset introduced. The magnitude of their opportunity cost, though, differs from that of Simaan's. Tew, Reid and Witt also found that as the number of assets in the portfolio increases the opportunity cost decreases. It is hard to comment on that conclusion because even though I did consider portfolios of different sizes (26-asset portfolio and nine-asset portfolio), both my portfolios include a nominally risk-free asset that was absent from any of Tew, Reid and Witt's portfolios.

Table 1 and Table 2 also show that as the level of relative risk aversion increases the standard deviations of the proportionate opportunity costs decrease: the distributions of the opportunity cost are getting "tighter". As the level of relative risk aversion 
increases more and more of the numerical values for the opportunity costs are concentrating around their means. Thus we see that as the level of risk aversion increases, as investors become less risk tolerant, the perceptions of the optimal constrained meanvariance portfolio strategy for investors with different asset sets are more similar to each other than perceptions of that strategy for different investors with lower risk aversion.

For 26 assets, Table 1 shows that the lowest mean (over 1,000 replications) of the proportionate opportunity cost, $0.5 \%(0.005)$, corresponds to the high level or risk aversion of 31 . This means that an investor with the level of relative risk aversion of 31 being unconstrained will be equally happy as if he was constrained but had $0.5 \%$ more of initial wealth. The highest mean (over 1,000 replications) of the proportionate opportunity cost, $5.6 \%(0.056)$, corresponds to the very low level of relative risk aversion of 0.7 . This means that an investor with the level of relative risk aversion of 0.7 being unconstrained will be equally happy as if he was constrained but had $5.6 \%$ more of initial wealth.

For low levels (from 3 to 0.7 ) of relative risk aversion the mean (over 1,000 replications) of the proportionate opportunity cost ranges from $3.2 \%(0.032)$ for relative risk aversion of 3 to $5.6 \%(0.056)$ for relative risk aversion of 0.7 .

For medium (from 12 to 9) levels of relative risk aversion the mean (over 1,000 replications) of the proportionate opportunity cost ranges from 1.9\% (0.019) for relative risk aversion of 12 to $3.0 \%(0.030)$ for relative risk aversion of 9 . This suggests that even medium risk-tolerance investors value optimal unconstrained portfolios high enough as oppose to constrained investment behavior to require from $1.9 \%$ to $3.0 \%$ of additional initial wealth to stay constrained. 
For nine assets, Table 2 shows that the lowest mean (over 1,000 replications) of the proportionate opportunity cost, $0.4 \%(0.004)$, corresponds to the high level of risk aversion of 31 . This means that an investor with the level of relative risk aversion of 31 being unconstrained will be equally happy as if he was constrained but had $0.4 \%$ more of initial wealth. The highest mean (over 1,000 replications) of the proportionate opportunity cost, $3.5 \%(0.035)$, corresponds to the very low level of relative risk aversion of 0.7 . This means that an investor with the level of relative risk aversion of 0.7 being unconstrained will be equally happy as if he was constrained but had 3.5\% more of initial wealth.

The highest values of the proportionate opportunity cost (the means over 1,000 replications) correspond to low levels of relative risk aversion (from 0.7 to 3 ) and range from $1.1 \%(0.011)$ for relative risk aversion of 3 to $3.5 \%(0.035)$ for relative risk aversion of 0.7. Investors with low levels of risk aversion (from 3 to 0.7 ) in the presence of nine assets will require from $1.1 \%$ to $3.5 \%$ of initial wealth to stay constrained and accept the mean-variance constrained optimal portfolio. These magnitudes, as a matter of fact, are lower than those for 26 assets in Table 1. This suggests that in the presence of a greater number of available assets investors find broader and more daring investment strategies that are further away from the mean-variance efficient one, and correspond to a higher proportionate opportunity cost.

For medium (from 12 to 9) levels of relative risk aversion the mean (over 1,000 replications) of the proportionate opportunity cost ranges from $0.6 \%(0.006)$ for relative risk aversion of 12 to $0.9 \%(0.009)$ for relative risk aversion of 9 . These numbers are about three times as small as those for 26 assets. 
These two tables suggest that the more assets are available for investors the further away low risk aversion investors will go from the mean-variance constrained strategy.

\subsubsection{Optimal Portfolio Shares}

Table 3 and Table 4 present typical optimal portfolio shares for unconstrained and constrained portfolio strategies for three different levels of relative risk aversion: low (of 0.7), medium (of 11) and high (of 31), for 26 assets and for nine assets, in each case for a different set of available assets giving an opportunity cost that is typical for that level of risk aversion.

For both tables for risk aversion of 0.7 more than $100 \%$ of initial wealth, $w_{0}$, is held in the nominally risky assets (asset \#1 through asset \#25 in Table 3 and asset \#1 through asset \#8 in Table 4) as a group, and Treasury bills are held in negative quantities.

As risk aversion increases, as investors become more conservative and less risktolerant, the proportion of initial wealth held in Treasury bills increases, and correspondingly the proportion of initial wealth held in the group of nominally risky assets decreases.

The tables show that unconstrained (and constrained) optimal portfolio shares are not similar for different levels of risk aversion. As a matter of fact, optimal unconstrained and constrained portfolios for the low level of relative risk aversion of 0.7 have more extreme quantities (negative as well as positive) of assets than optimal unconstrained and 
Table 3

Illustrative optimal portfolio shares for unconstrained and mean-variance constrained portfolio strategies for different values of relative risk aversion for 26 $\operatorname{assets}^{1}$

\begin{tabular}{|c|c|c|c|c|c|c|}
\hline \multirow[t]{2}{*}{$\begin{array}{c}\text { \# of } \\
\text { An Asset }\end{array}$} & \multicolumn{2}{|c|}{$\begin{array}{l}\text { Relative Risk Aversion, }(1-\gamma) \text {, } \\
\text { equal to } 0.7\end{array}$} & \multicolumn{2}{|c|}{$\begin{array}{c}\text { Relative Risk Aversion, } \\
(1-\gamma) \text {, equal to } 11\end{array}$} & \multicolumn{2}{|c|}{$\begin{array}{c}\text { Relative Risk Aversion, } \\
(1-\gamma) \text {, equal to } 31\end{array}$} \\
\hline & Unconstrained & Constrained & Unconstrained & Constrained & Unconstrained & Constrained \\
\hline 1 & 0.679 & 0.381 & -0.022 & -0.022 & -0.002 & 0.001 \\
\hline 2 & 2.168 & 1.635 & 0.076 & 0.065 & 0.000 & -0.006 \\
\hline 3 & 0.525 & 0.445 & 0.016 & 0.037 & 0.021 & 0.017 \\
\hline 4 & 2.884 & 3.649 & 0.283 & 0.289 & 0.003 & 0.006 \\
\hline 5 & 0.239 & 0.327 & 0.043 & 0.042 & 0.179 & 0.148 \\
\hline 6 & 1.022 & 0.808 & 0.053 & 0.062 & 0.056 & 0.076 \\
\hline 7 & 0.360 & 0.470 & -0.033 & -0.086 & -0.018 & -0.013 \\
\hline 8 & -2.332 & -2.483 & 0.121 & 0.112 & 0.033 & 0.025 \\
\hline 9 & -1.082 & -0.795 & 0.050 & 0.068 & 0.094 & 0.079 \\
\hline 10 & 4.884 & 2.855 & 0.046 & 0.060 & -0.046 & -0.019 \\
\hline 11 & 0.363 & 0.533 & -0.012 & -0.014 & -0.119 & -0.100 \\
\hline 12 & -4.082 & -2.547 & -0.005 & -0.061 & 0.012 & 0.015 \\
\hline 13 & 4.073 & 6.576 & -0.006 & -0.016 & -0.058 & -0.074 \\
\hline 14 & -0.419 & -0.995 & -0.035 & -0.061 & -0.025 & -0.018 \\
\hline 15 & -0.879 & -1.147 & 0.078 & 0.219 & 0.324 & 0.292 \\
\hline 16 & 1.216 & 1.457 & -0.118 & -0.094 & 0.035 & 0.026 \\
\hline 17 & 4.971 & 5.163 & 0.151 & 0.126 & 0.000 & 0.000 \\
\hline 18 & 3.195 & 3.513 & 0.090 & 0.130 & -0.127 & -0.085 \\
\hline 19 & 2.554 & 0.649 & -0.154 & -0.147 & -0.023 & -0.018 \\
\hline 20 & -3.750 & -1.816 & -0.031 & -0.013 & -0.005 & -0.011 \\
\hline 21 & -0.014 & 0.129 & -0.158 & -0.175 & 0.000 & -0.001 \\
\hline 22 & -0.728 & 0.154 & -0.027 & -0.026 & 0.041 & 0.040 \\
\hline 23 & -1.793 & -2.595 & 0.035 & 0.036 & 0.046 & 0.032 \\
\hline 24 & 1.913 & 0.926 & 0.452 & 0.332 & 0.021 & 0.027 \\
\hline 25 & -0.070 & 0.333 & -0.111 & -0.089 & 0.004 & -0.005 \\
\hline $26^{2}$ & -14.896 & -16.626 & 0.218 & 0.225 & 0.552 & 0.564 \\
\hline $\mathrm{E}\left(\mathrm{X}^{* \prime} \widetilde{R}\right)^{3}$ & 1.383 & 1.358 & 1.033 & 1.025 & 1.007 & 1.002 \\
\hline $\begin{array}{l}\text { Certainty } \\
\text { Equivalent }\end{array}$ & 1.209 & 1.145 & 1.022 & 0.998 & 1.001 & 0.996 \\
\hline
\end{tabular}

${ }^{\prime}$ Numbers are not comparable across levels of risk aversion, because for each level of risk aversion a different set of available assets was used: a set giving an exact value of opportunity cost typical for that level of risk aversion.

${ }^{2}$ The $26^{\text {th }}$ asset is risk-free in nominal terms.

${ }^{3}$ Monthly gross expected returns on portfolios. 
Table 4

Illustrative optimal portfolio shares for unconstrained and mean-variance constrained portfolio strategies for different values of relative risk aversion for nine $\operatorname{assets}^{1}$

\begin{tabular}{|c|c|c|c|c|c|c|}
\hline \multirow[t]{2}{*}{$\begin{array}{c}\text { \# of } \\
\text { An Asset }\end{array}$} & \multicolumn{2}{|c|}{$\begin{array}{l}\text { Relative Risk Aversion, }(1-\gamma) \text {, } \\
\text { equal to } 0.7\end{array}$} & \multicolumn{2}{|c|}{$\begin{array}{c}\text { Relative Risk Aversion, } \\
(1-\gamma) \text {, equal to } 11\end{array}$} & \multicolumn{2}{|c|}{$\begin{array}{l}\text { Relative Risk Aversion, } \\
(1-\gamma) \text {, equal to } 31\end{array}$} \\
\hline & Unconstrained & Constrained & Unconstrained & Constrained & Unconstrained & Constrained \\
\hline 1 & 0.270 & 0.229 & 0.292 & 0.253 & 0.027 & 0.021 \\
\hline 2 & 0.004 & 0.262 & 0.100 & 0.074 & -0.051 & -0.056 \\
\hline 3 & -0.199 & 0.037 & 0.590 & 0.514 & 0.038 & 0.027 \\
\hline 4 & 0.598 & 0.252 & 0.110 & 0.127 & 0.022 & 0.047 \\
\hline 5 & 0.001 & 0.015 & 0.162 & 0.082 & -0.006 & -0.003 \\
\hline 6 & 0.472 & 0.786 & -0.536 & -0.450 & 0.040 & 0.037 \\
\hline 7 & 0.231 & 0.202 & -0.306 & -0.329 & 0.013 & 0.003 \\
\hline 8 & 5.234 & 3.659 & 0.280 & 0.242 & 0.055 & 0.047 \\
\hline $9^{2}$ & -5.612 & -4.442 & 0.307 & 0.487 & 0.862 & 0.876 \\
\hline $\mathrm{E}\left(\mathrm{X}^{* \prime} \widetilde{R}\right)^{3}$ & 1.079 & 1.058 & 1.027 & 1.018 & 1.004 & 1.001 \\
\hline $\begin{array}{c}\text { Certainty } \\
\text { Equivalent }\end{array}$ & 1.056 & 1.020 & 1.016 & 1.009 & 1.002 & 0.998 \\
\hline
\end{tabular}

${ }^{\prime}$ Numbers are not comparable across levels of risk aversion, because for each level of risk aversion a different set of available assets was used: a set giving an exact value of opportunity cost typical for that level of risk aversion.

${ }^{2}$ The $9^{\text {th }}$ asset is risk-free in nominal terms.

${ }^{3}$ Monthly gross expected returns on portfolios.

constrained portfolios for medium level of relative risk aversion of 11 and for high level of relative risk aversion of 31. Extremely negative quantities of assets for high risktolerance investors mean that the investors follow an aggressive short sale strategy.

The extremely negative quantities of assets in portfolios for low risk aversion of 0.7 confirm Simaan (1993), who found exactly the same thing: investors with low levels of risk aversion follow very aggressive short sale strategies.

Also Table 3 and Table 4 show monthly expected returns on unconstrained and constrained optimal portfolios, $E\left(X^{* \prime} \widetilde{R}\right)$, for the three levels of relative risk aversion $(0.7,11$ and 31$)$. 
The expected returns for constrained and unconstrained optimal portfolios for risk aversion of 0.7 are very dramatic for 26 -asset portfolios and large for nine-asset portfolios. Expected returns are of large size for risk aversion of 11 and of small size for risk aversion of 31. Such extreme magnitudes of expected portfolio returns for high risktolerance investors confirm the previously made conclusion about very aggressive short sale strategies. With initial wealth set equal to $l$ these magnitudes suggest very leveraged portfolios (unconstrained as well as constrained). For investors with risk aversion of 11 and 31 there is, definitely, some short selling is going on too, but not as aggressive as for investors with risk aversion of 0.7. The less aggressive short selling for medium or high risk aversion leads to lower mean return portfolios. Simaan's expected portfolio returns are much lower that the ones I have predicted for 26 -asset portfolios, but only a little bit lower than those for nine-asset portfolios.

The big difference between the expected portfolio returns with 26 assets and those with nine assets is due to the fact that the more assets are available for investors the more opportunities they have to seek higher mean while simultaneously increasing diversification.

In comparing unconstrained expected portfolio returns and constrained expected portfolio returns for the three levels of risk aversion for the two tables I find that unconstrained and constrained expected portfolio returns for risk aversion of 31 are very close to each other; for risk aversion of 11 they are somewhat close, but not very; for risk aversion of 0.7 unconstrained and constrained expected portfolio returns are not close at all. These unconstrained and constrained expected portfolio returns show that as risk aversion increases, the closer to each other expected returns on unconstrained and 
constrained portfolios are, and thus the more nearly indifferent an investor is between the unconstrained and constrained portfolio strategies.

It is also interesting to compare expected monthly portfolio returns from Table 3 and Table 4 and the mean values of the proportionate opportunity cost reported in Table 1 and Table 2. For 26 assets, as Table 1 shows, the mean over 1,000 replications of the proportionate opportunity cost reaches $5.6 \%$ for unconstrained investors with the net expected monthly portfolio return (gross expected monthly portfolio return minus 1.0, multiplied by $100 \%$ ) of $38.3 \%$ for the level of risk aversion of 0.7 . For risk aversion of 31 the mean of the proportionate opportunity cost for unconstrained investors and the expected monthly portfolio return are almost the same $(0.5 \%$ for the mean of the proportionate opportunity cost and $0.7 \%$ for the net expected portfolio return).

For nine assets, as Table 2 shows, the mean over 1,000 replications of the proportionate opportunity cost reaches $3.5 \%$ for unconstrained investors with expected net monthly portfolio return of $7.9 \%$ for the risk aversion of 0.7 . As the level of risk aversion increases to 31 the mean of the proportionate opportunity cost and the net expected monthly portfolio return become exactly the same, $0.4 \%$.

Table 3 and Table 4 also report the certainty equivalents calculated for the same three levels of relative risk aversion $(0.7,11$ and 31$)$. The certainty equivalent, $(C E)$, is defined by

$$
\frac{1}{\gamma} C E^{\gamma}=\frac{1}{\gamma} w_{0}^{\gamma} E\left(\widetilde{R}^{\gamma}\right)
$$

and so, with $w_{0}=1$,

$$
C E=\left(E\left[\widetilde{R}^{\gamma}\right]\right)^{\frac{1}{\gamma}}
$$


The certainty equivalent represents the amount of certain wealth that would be viewed with indifference to the optimal portfolio. It is computed for investors of different levels of risk aversion: low (of 0.7), medium (of 11) and high (of 31). The two tables show that as risk aversion increases the value of certainty equivalent decreases (for the unconstrained portfolio strategy as well as for the constrained). This suggests that as investors become more afraid of risk they use less risky portfolio strategies and will be expecting lower returns from those portfolios and, so, the certain amount of wealth they will be willing to accept with indifference will decrease.

It is interesting to compare certainty equivalents for unconstrained optimal portfolio strategies from Table 3 and Table 4 and the mean values of the proportionate opportunity cost from Table 1 and Table 2 . For 26 assets, as Table 1 shows, the mean over 1,000 replications of the proportionate opportunity cost reaches $5.6 \%$ for unconstrained investors with the level of risk aversion of 0.7 whose net certainty equivalent (certainty equivalent minus 1.0 ) equals $20.9 \%$. As the level of risk aversion increases to 31 the mean of the proportionate opportunity cost falls to $0.5 \%$ while the net certainty equivalent falls to $0.1 \%$.

For nine assets, as Table 2 shows, the mean value of the proportionate opportunity cost reaches 3.5\% for unconstrained investors with their net certainty equivalent being equal to $5.6 \%$ for the risk aversion of 0.7 . As the level of risk aversion increases to 31 the mean of the proportionate opportunity cost for unconstrained investors falls to $0.4 \%$ while the net certainty equivalent falls to $0.2 \%$.

Table 5 presents the percentage of gross certainty equivalent for unconstrained portfolio strategies lost due to the mean-variance constraint, computed as shown in (18). 
The percentage of gross certainty equivalent lost due to the constraint is timeless just like the proportionate opportunity cost, $\theta-1.0$.

$$
\text { The percentage loss }=\frac{C E^{\text {Uncd }}-C E^{\text {Const }}}{C E^{\text {Uncd }}}=\frac{\theta-1.0}{\theta}
$$

The highest percentage loss, $5.3 \%$, happens for the investors with risk aversion of 0.7 holding 26 assets in their portfolios. As risk aversion increases, for both 26-asset and nine-asset portfolios, the percentage loss decreases. The lowest percentage loss, $0.4 \%$, is observed for the investors with risk aversion of 31 holding nine assets in their portfolios. Table 5 confirms the previously made conclusion that as investors become more afraid of risk their perceptions of the optimal constrained and optimal unconstrained portfolio strategies become more and more similar. Hence, as the level of risk aversion increases the certain amount of wealth unconstrained investors and constrained investors will be willing to accept with indifference will be getting closer to each other, and, therefore, the percentage loss in certainty equivalents due to the constraint will decrease.

\section{Table 5}

The percentage of certainty equivalent lost due to the mean-variance constraint for various levels of relative risk aversion with no exaggeration of extreme returns

\begin{tabular}{|c|c|c|c|c|c|c|c|c|c|c|c|c|}
\hline Number of & \multicolumn{10}{c|}{ Relative } & Risk & Aversion, \\
\cline { 2 - 13 } assets & 0.7 & 1 & 2 & 3 & 9 & 10 & 11 & 12 & 29 & 30 & 31 \\
\hline 26 assets & 5.3 & 4.6 & 3.4 & 3.1 & 2.9 & 2.7 & 2.3 & 1.9 & 1.2 & 0.6 & 0.5 \\
9 assets & 3.4 & 2.7 & 1.4 & 1.1 & 0.9 & 0.7 & 0.7 & 0.6 & 0.5 & 0.4 & 0.4 \\
\hline
\end{tabular}

The percentage loss $=\frac{C E^{\text {Uncd }}-C E^{\text {Cond }}}{C E^{\text {Uncd }}} * 100 \%=\frac{\theta-1}{\theta} * 100 \%$.

Table 3 and Table 4 show that magnitudes of portfolio shares for different levels of relative risk aversion, as well as for unconstrained and constrained portfolio strategies, are very different. Comparison of optimal portfolio shares across different levels of risk 
aversion is meaningless, since portfolio shares for different levels of relative risk aversion were calculated by using different sets of available assets for each level of risk aversion. But comparison of unconstrained and constrained optimal portfolios is very interesting (how similar are constrained and unconstrained asset holdings to each other?) and possible (constrained and unconstrained portfolio shares for a particular level of relative risk aversion correspond to the same set of available assets).

In terms of comparing unconstrained and constrained optimal portfolios, I find in Table 3 and Table 4 that absolute values of shares of unconstrained portfolios are, in almost all cases, bigger than those of constrained portfolios.

Table 6 and Table 7 present correlation coefficients and geometric distances calculated between unconstrained and constrained portfolio share vectors for different levels of relative risk aversion: low, (from 0.7 to 3), medium (from 9 to 12) and high (from 29 to 31), for all assets in ones' portfolio (26 and nine) and separately for the group of nominally risky assets only (asset \#1 through asset \#25 in Table 6 and asset \#1 through asset \#8 in Table 7).

Both tables show high correlation between unconstrained and constrained optimal portfolio shares for all levels of risk aversion. But correlation coefficients calculated for all assets in portfolios (26 or nine) are higher that those calculated for the nominally risky assets only.

This difference in correlation coefficients can be explained by the presence of Treasury bills in optimal portfolios of 26 and nine assets. For the low levels of risk aversion when investors use highly leveraged portfolios they go very short on Treasury bills (in constrained as well as unconstrained strategies), which results in very similar 
Table 6

Illustrative correlation coefficients and geometric distances for unconstrained and mean-variance constrained portfolios for different levels of relative risk aversion for 26 assets $^{1}$

\begin{tabular}{|c|c|c|c|c|}
\hline $\begin{array}{c}\text { Relative } \\
\text { Risk } \\
\text { Aversion, }(1-\gamma)\end{array}$ & $\begin{array}{c}\text { Correlation } \\
\text { Coefficients } \\
\text { for all 26 assets }\end{array}$ & $\begin{array}{c}\text { Geometric } \\
\text { Distance } \\
\text { for 26 assets }\end{array}$ & $\begin{array}{c}\text { Correlation Coefficients for } \\
\text { the group of nominally risky } \\
\text { Assets only (first 25 assets) }\end{array}$ & $\begin{array}{c}\text { Geometric Distance }{ }^{2} \text { for } \\
\text { nominally risky assets only } \\
\text { (first 25 assets) }\end{array}$ \\
\hline 0.7 & 0.967 & 7.382 & 0.907 & 7.374 \\
1 & 0.923 & 5.240 & 0.912 & 4.945 \\
2 & 0.968 & 2.068 & 0.869 & 1.945 \\
3 & 0.958 & 2.046 & 0.964 & 1.906 \\
9 & 0.976 & 0.459 & 0.969 & 0.438 \\
10 & 0.926 & 0.301 & 0.921 & 0.287 \\
11 & 0.946 & 0.215 & 0.941 & 0.209 \\
12 & 0.962 & 0.169 & 0.956 & 0.140 \\
29 & 0.978 & 0.139 & 0.947 & 0.134 \\
30 & 0.983 & 0.084 & 0.965 & 0.076 \\
31 & 0.993 & 0.082 & 0.989 & 0.081 \\
\hline
\end{tabular}

${ }^{1}$ Calculated for different fixed sets of assets for different levels of risk aversion; in each case the asset set is the one giving an opportunity cost typical for that level of risk aversion.

${ }^{2}$ Geometric distance is defined as $\left[(X-\hat{X})^{\prime}(X-\hat{X})\right]^{1 / 2}$ where $X$ is the unconstrained portfolio, and $\hat{X}$ is the constrained portfolio.

Table 7

Illustrative correlation coefficients and geometric distances for unconstrained and mean-variance constrained portfolios for different levels of relative risk aversion for nine assets ${ }^{1}$

\begin{tabular}{|c|c|c|c|c|}
\hline $\begin{array}{c}\text { Relative } \\
\text { Risk } \\
\text { Aversion, }(1-\gamma)\end{array}$ & $\begin{array}{c}\text { Correlation } \\
\text { Coefficients } \\
\text { for all 9 assets }\end{array}$ & $\begin{array}{c}\text { Geometric } \\
\text { Distance } \\
\text { for 9 assets }\end{array}$ & $\begin{array}{c}\text { Correlation Coefficients for } \\
\text { the group of nominally risky } \\
\text { assets only (first 8 assets) }\end{array}$ & $\begin{array}{c}\text { Geometric Distance for } \\
\text { nominally risky assets only } \\
\text { (first 8 assets) }\end{array}$ \\
\hline 0.7 & 0.996 & 5.835 & 0.978 & 2.788 \\
1 & 0.993 & 2.048 & 0.987 & 1.682 \\
2 & 0.985 & 0.619 & 0.978 & 0.409 \\
3 & 0.813 & 0.409 & 0.756 & 0.408 \\
9 & 0.997 & 0.086 & 0.986 & 0.047 \\
10 & 0.998 & 0.047 & 0.972 & 0.032 \\
11 & 0.969 & 0.037 & 0.954 & 0.035 \\
12 & 0.996 & 0.040 & 0.991 & 0.040 \\
29 & 0.956 & 0.026 & 0.950 & 0.020 \\
30 & 0.890 & 0.021 & 0.879 & 0.018 \\
31 & 0.989 & 0.017 & 0.976 & 0.016 \\
\hline
\end{tabular}

${ }^{1}$ Calculated for different fixed sets of assets for different levels of risk aversion; in each case the asset set is the one giving an opportunity cost typical for that level of risk aversion.

${ }^{2}$ Geometric distance is defined as $\left[(X-\hat{X})^{\prime}(X-\hat{X})\right]^{1 / 2}$ where $X$ is the unconstrained portfolio, and $\hat{X}$ is the constrained portfolio. 
negative portfolio shares for the asset. This similarity has a strong effect on the correlation calculated over all assets shares including the Treasury bills share.

The geometric distance calculated for different values of risk aversion is another way to compare unconstrained and constrained portfolios. Geometric distance is defined

as $\left[(X-\hat{X})^{\prime}(X-\hat{X})\right]^{1 / 2}$ where $X$ is the unconstrained portfolio, and $\hat{X}$ is the constrained portfolio. The greater the geometric distance between the two portfolios, in other words the further the unconstrained optimal portfolio is from the constrained optimal portfolio, the greater an investor's welfare loss is likely to be if he must choose the constrained portfolio, and so the higher the opportunity cost for the investor is. Table 6 and Table 7 show that as risk aversion increases, as investors become less risk tolerant, the geometric distance between unconstrained and constrained portfolios decreases making unconstrained and constrained portfolios more similar to each other. Simaan reached the same conclusion for the case with the riskless asset though his geometric distances are greater in magnitude than mine for my nine-asset portfolios and somewhat close to mine for my 26-asset portfolios. So, if the unconstrained optimal portfolio and the constrained optimal portfolio are getting closer as risk aversion increases, it must be that the opportunity cost will decrease. That is exactly my finding from Table 1 and Table 2.

\subsubsection{Regret in the Worst-Case Scenario}

Large negative and positive asset holdings (Table 3 and Table 4) in portfolios for investors with a level of risk aversion of 0.7 suggest that the investors take on a lot of risk. This raises the question: if the worst possible portfolio outcome occurs, then how 
much will the investors suffer from such an outcome? It is possible to measure the investors' proportionate regret from the worst-case scenario with such a risky portfolio.

Table 8 and Table 9 report the proportionate regret, $(\theta-1)$, for 26 assets and for nine assets, that will be incurred by investors if the worst possible outcome of asset returns occurs. This $\theta$ is defined by

$$
U\left(\theta\left(X^{* \prime} R\right)^{\text {worst }}\right)=E U\left(X^{* \prime} \widetilde{R}\right)
$$

where $X^{*}$ is the optimally chosen portfolio, $\left(X^{* \prime} R\right)^{\text {worst }}$ is the one of the 120 states of nature giving the lowest portfolio return, $U\left[\left(X^{* \prime} R\right)^{\text {worst }}\right]$ is an investor's utility from getting the worst possible portfolio outcome, $E U\left(X^{* \prime} \widetilde{R}\right)$ is an investor's ex ante expected utility.

For unconstrained investors (for the case with 26 assets as well as for nine assets) the mean of the proportionate regret (over 1,000 replications) is the highest for the low level of risk aversion of 0.7 and the lowest for the high level of risk aversion of 31 . This means that high risk-tolerance investors do choose risky unconstrained asset allocations. And it is getting riskier as the number of assets increases. Those asset allocations are so risky at the level of risk aversion of 0.7 , that if the worst possible outcome occurs it would require for investors with 26 assets to receive $1022.1 \%$ of initial wealth in compensation and for investors with nine assets to receive $525.7 \%$ of initial wealth in order to get the same level of ex post utility as their ex ante expected utility. For the high level of 31 for risk aversion the mean of the proportionate regret (over 1,000 replications) is $4.1 \%(0.041)$ for investors with 26 assets and 2.7\% $(0.027)$ for investors with nine assets. Such a low proportionate regret suggests that low risk-tolerance unconstrained investors choose very conservative unconstrained asset allocations. So conservative are 
Table 8

The ex post proportionate regret, $(\theta-1)$, under the worst portfolio outcome for 26 assets for unconstrained and mean-variance constrained portfolios

\begin{tabular}{|c|c|c|c|c|c|c|}
\hline $\begin{array}{l}\text { Relative Risk } \\
\text { Aversion, }(1-\gamma)\end{array}$ & Portfolios & Smallest & Mean & Median & Largest & Standard Deviation \\
\hline 0.7 & $\begin{array}{c}\text { Unconstrained } \\
\text { Constrained }\end{array}$ & $\begin{array}{l}0.146 \\
0.112 \\
\end{array}$ & $\begin{array}{r}10.221 \\
4.838 \\
\end{array}$ & $\begin{array}{l}9.310 \\
3.083 \\
\end{array}$ & $\begin{array}{r}41.766 \\
16.435 \\
\end{array}$ & $\begin{array}{r}14.551 \\
7.772 \\
\end{array}$ \\
\hline 11 & $\begin{array}{c}\text { Unconstrained } \\
\text { Constrained }\end{array}$ & $\begin{array}{l}0.058 \\
0.049 \\
\end{array}$ & $\begin{array}{l}0.135 \\
0.113 \\
\end{array}$ & $\begin{array}{l}0.120 \\
0.111 \\
\end{array}$ & $\begin{array}{l}0.243 \\
0.155 \\
\end{array}$ & $\begin{array}{l}0.024 \\
0.019 \\
\end{array}$ \\
\hline 31 & $\begin{array}{c}\text { Unconstrained } \\
\text { Constrained }\end{array}$ & $\begin{array}{l}0.023 \\
0.012\end{array}$ & $\begin{array}{l}0.041 \\
0.037\end{array}$ & $\begin{array}{l}0.040 \\
0.035\end{array}$ & $\begin{array}{l}0.076 \\
0.054\end{array}$ & $\begin{array}{l}0.007 \\
0.006\end{array}$ \\
\hline
\end{tabular}

Table 9

The ex post proportionate regret, $(\theta-1)$, under the worst portfolio outcome for nine assets for unconstrained and mean-variance constrained portfolios

\begin{tabular}{|c|c|c|c|c|c|c|}
\hline $\begin{array}{c}\text { Relative Risk } \\
\text { Aversion, (1- } \gamma)\end{array}$ & Portfolios & Smallest & Mean & Median & Largest & Standard Deviation \\
\hline $\mathbf{0 . 7}$ & Unconstrained & 0.162 & 5.257 & 4.425 & 25.509 & 3.148 \\
& Constrained & 0.103 & 3.489 & 2.916 & 9.346 & 2.654 \\
\hline $\mathbf{1 1}$ & Unconstrained & 0.011 & 0.069 & 0.061 & 0.240 & 0.018 \\
& Constrained & 0.009 & 0.048 & 0.039 & 0.153 & 0.017 \\
\hline \multirow{3}{*}{$\mathbf{3 1}$} & Unconstrained & 0.009 & 0.027 & 0.025 & 0.069 & 0.007 \\
& Constrained & 0.008 & 0.019 & 0.017 & 0.048 & 0.006 \\
\hline
\end{tabular}


their allocations that even the worst possible outcome will require for them less than $5.0 \%$ of initial wealth to get to the same level of utility as their ex ante expected utility.

For constrained portfolio strategies the mean proportionate regret (over 1,000 replications) ranges from $483.8 \%$ (4.838) for risk aversion of 0.7 to $3.7 \%$ (0.037) for risk aversion of 31 for investors with 26 assets, and from $348.9 \%$ (3.489) for risk aversion of 0.7 to $1.9 \%(0.019)$ for risk aversion of 31 for investors with nine assets. This means that constrained portfolios have a very restrictive character and do not let high risk-tolerance investors take a lot of risk. For low risk-tolerance investors constrained portfolios are somewhat close to unconstrained portfolios (the difference in the mean regret is less than $1 \%$ ) and represent very conservative asset allocations with very little risk to take. Note that the tendency for the mean-variance efficiency constraint to make portfolios more conservative is also seen in Table 3 and Table 4, which show that at each level of risk aversion the mean portfolio return is less when the constraint is present than when it is not.

\subsection{Historical Data with Extreme Values Exaggerated}

In order to check the robustness of the estimates of the proportionate opportunity cost I include extremely high and extremely low simulated asset returns in each data set of available assets (26 assets as well as nine assets).

I construct the simulated extremely high and extremely low asset returns the following way. For the original data set for each historical time period I compute the average excess return across all assets in the data set. A historical time period with the highest average excess return across all assets defines the historical period with the 
highest returns. A historical time period with the lowest average excess return across all assets defines the historical period with the lowest returns. Then, for the extreme historical periods only I calculate the deviation of each asset's return from that asset's return intertemporal mean return. The deviations are doubled and then added back to assets' intertemporal means. This way I create two fictional time periods with exaggerated high and exaggerated low returns. These fictional asset returns provide simulated extreme time periods to replace the time periods they were constructed from. The rest of the original data set remains unchanged.

Then I repeat the whole procedure of calculating the proportionate opportunity cost 1,000 times in each case simulating two fictional data periods. This gives me 1,000 new $\theta$ 's from the 1,000 data sets with extreme returns added. The results from this project are reported in Table 10 for 26 assets and 11 for nine assets.

Out of all values considered for relative risk aversion, the lowest mean (over 1,000 replications) of the proportionate opportunity cost for 26 assets and for nine assets corresponds to the high level of relative risk aversion of 31 . The highest mean (over 1,000 replications) of the proportionate opportunity cost for 26 assets and for nine assets corresponds to the low level of relative risk aversion of 0.7 .

The results in the second project confirm the results from the first one: as the level of relative risk aversion increases the proportionate opportunity cost decreases, the better the mean-variance efficient portfolio performs ex ante and the lower the proportion of initial wealth an investor requires to stay constrained and accept the mean-variance efficient portfolio instead of the optimal unconstrained portfolio. Also, the same way as with the original data set, as the level of relative risk aversion increases the standard 
Table 10

The proportionate opportunity cost of the mean-variance constraint, $(\theta-1)$, for various values of relative risk aversion with extreme returns exaggerated for 26

assets

\begin{tabular}{|c|c|c|c|c|c|}
\hline $\begin{array}{c}\text { Relative } \\
\text { Risk Aversion, (1- } \gamma)\end{array}$ & Smallest & Mean & Median & Largest & Standard Deviation \\
\hline \multicolumn{6}{|l|}{ Low } \\
\hline 0.7 & 0.021 & 0.108 & 0.091 & 0.875 & 0.198 \\
\hline 1 & 0.004 & 0.097 & 0.090 & 0.769 & 0.098 \\
\hline 2 & 0.002 & 0.078 & 0.076 & 0.701 & 0.065 \\
\hline 3 & 0.002 & 0.060 & 0.058 & 0.643 & 0.050 \\
\hline \multicolumn{6}{|l|}{ Medium } \\
\hline 9 & 0.001 & 0.053 & 0.049 & 0.550 & 0.047 \\
\hline 10 & 0.001 & 0.049 & 0.041 & 0.245 & 0.045 \\
\hline 11 & 0.000 & 0.042 & 0.035 & 0.180 & 0.037 \\
\hline 12 & 0.000 & 0.038 & 0.034 & 0.124 & 0.031 \\
\hline \multicolumn{6}{|l|}{ High } \\
\hline 29 & 0.000 & 0.028 & 0.026 & 0.086 & 0.028 \\
\hline 30 & 0.000 & 0.019 & 0.019 & 0.081 & 0.026 \\
\hline 31 & 0.000 & 0.015 & 0.014 & 0.042 & 0.022 \\
\hline
\end{tabular}

Table 11

The proportionate opportunity cost of the mean-variance constraint, $(\theta-1)$, for various values of relative risk aversion with extreme returns exaggerated for nine

assets

\begin{tabular}{|c|c|c|c|c|c|}
\hline $\begin{array}{c}\text { Relative } \\
\text { Risk Aversion, (1- } \gamma)\end{array}$ & Smallest & Mean & Median & Largest & Standard Deviation \\
\hline \multicolumn{6}{|l|}{ Low } \\
\hline 0.7 & 0.002 & 0.064 & 0.060 & 0.737 & 0.195 \\
\hline 1 & 0.002 & 0.041 & 0.038 & 0.719 & 0.082 \\
\hline 2 & 0.001 & 0.025 & 0.020 & 0.614 & 0.041 \\
\hline 3 & 0.001 & 0.015 & 0.011 & 0.591 & 0.037 \\
\hline \multicolumn{6}{|l|}{ Medium } \\
\hline 9 & 0.001 & 0.016 & 0.010 & 0.491 & 0.034 \\
\hline 10 & 0.000 & 0.015 & 0.010 & 0.411 & 0.031 \\
\hline 11 & 0.000 & 0.012 & 0.009 & 0.361 & 0.030 \\
\hline $\begin{array}{c}12 \\
\text { High }\end{array}$ & 0.000 & 0.011 & 0.009 & 0.321 & 0.028 \\
\hline 29 & 0.000 & 0.009 & 0.006 & 0.292 & 0.019 \\
\hline 30 & 0.000 & 0.008 & 0.005 & 0.288 & 0.015 \\
\hline 31 & 0.000 & 0.008 & 0.005 & 0.270 & 0.015 \\
\hline
\end{tabular}


deviation of the proportionate opportunity cost decreases. This means that as the level of risk aversion increases, as investors facing different asset sets become less risk tolerant, their perceptions of the optimal constrained mean-variance portfolio strategy, even with extreme historical periods, are more similar to each other than perceptions of the strategy for investors with lower risk aversion.

The only difference between Tables 1-2 and Tables 10-11 is that the magnitude of the proportionate opportunity cost is bigger when extreme returns are exaggerated. So, the presence of exaggerated extreme returns in the probability distribution moves meanvariance portfolio optimization further from optimal and the decision of choosing the mean-variance constrained portfolio over the unconstrained portfolio is more costly. This makes sense because of the following. The mean-variance approach is optimal only under normality (elliptical symmetry) and if the normality condition is violated, as it is in the historical asset data set with not exaggeration of extreme returns, then the mean-variance framework is not optimal, and there is a cost investors will be incurring by choosing it instead of the optimal portfolio strategy. The presence of exaggerated extreme returns in the historical distribution will move the distribution even further away from elliptical symmetry than it was with no exaggerated extreme returns. And this will increase the size of the cost that the investor will be incurring from choosing the mean-variance portfolio strategy instead of the optimal strategy.

The lowest mean (over 1,000 replications) of the proportionate opportunity cost for 26 assets (Table 10) is $1.5 \%(0.015)$ and corresponds to the level of risk aversion of 31. This means that an investor with the level of relative risk aversion of 31 being unconstrained will be equally happy as if he was constrained but had $1.5 \%$ more of initial 
wealth. The highest mean (over 1,000 replications) of the proportionate opportunity cost, $10.8 \%(0.108)$, corresponds to the very low level of relative risk aversion of 0.7 . This means that an investor with the level of relative risk aversion of 0.7 being unconstrained will be equally happy as if he was constrained but had $10.8 \%$ more of initial wealth.

The lowest mean (over 1,000 replications) of the proportionate opportunity cost for nine assets (Table 11) is $0.8 \%(0.008)$ and corresponds to the level of risk aversion of 31. The highest mean (over 1,000 replications) of the proportionate opportunity cost, $6.4 \%(0.064)$, corresponds to the very low level of relative risk aversion of 0.7 .

Table 12

The percentage of certainty equivalent lost due to the mean-variance constraint for various levels of relative risk aversion with extreme returns exaggerated

\begin{tabular}{|c|c|c|c|c|c|c|c|c|c|c|c|c|}
\hline Number of & \multicolumn{10}{c|}{ Relative Risk } & Aversion, $(1-\boldsymbol{\lambda})$ \\
\cline { 2 - 12 } assets & 0.7 & 1 & 2 & 3 & 9 & 10 & 11 & 12 & 29 & 30 & 31 \\
\hline 26 assets & 9.7 & 8.8 & 7.2 & 5.7 & 5.0 & 4.7 & 4.0 & 3.7 & 2.7 & 1.9 & 1.5 \\
9 assets & 6.0 & 3.9 & 2.4 & 1.5 & 1.5 & 1.4 & 1.2 & 1.1 & 0.9 & 0.8 & 0.7 \\
\hline
\end{tabular}

The percentage loss $=\frac{C E^{\text {Uncd }}-C E^{\text {Cond }}}{C E^{\text {Uncd }}} * 100 \%=\frac{\theta-1}{\theta} * 100 \%$.

Table 12 presents the percentage of gross certainty equivalent for unconstrained portfolio strategies lost due to the mean-variance constraint, computed as shown in (18), with extreme returns exaggerated in the asset return distribution. The percentage of gross certainty equivalent lost due to the constraint is timeless just like the proportionate opportunity cost, $\theta-1.0$.

The highest percentage loss, $9.7 \%$, happens for the investors with risk aversion of 0.7 holding 26 assets in their portfolios. As risk aversion increases, for both 26-asset and nine-asset portfolios, the percentage loss decreases. The lowest percentage loss, $0.7 \%$, is 
observed for the investors with risk aversion of 31 holding nine assets in their portfolios. Table 12 confirms the results from Table 5, and the previously made conclusion that as investors become more afraid of risk their perceptions of the optimal constrained and optimal unconstrained portfolio strategies become more and more similar. Hence, as the level of risk aversion increases the certain amount of wealth unconstrained investors as well as constrained investors will be willing to accept with indifference will be getting closer to each other, and, therefore, the percentage loss in certainty equivalents due to the constraint will decrease.

The only difference between Table 5 and Table 12 is that the magnitude of the percentage loss is bigger when extreme returns are exaggerated. This is another confirmation of the previously made conclusion that when extreme returns are exaggerated in the historical distribution it will move the distribution further away from

elliptical symmetry and increase the size of the certainty equivalent loss from choosing the mean-variance portfolio strategy instead of the optimal portfolio strategy.

\section{Conclusion}

In this essay I have investigated the opportunity cost incurred by investors when they use constrained optimal mean-variance efficient portfolios instead of unconstrained optimal portfolios. Two sets of returns have been used: the original historical returns and historical asset returns with extreme values exaggerated. CRRA utility function and the proportionate opportunity cost have been used. The opportunity cost has been calculated for different values of relative risk aversion (including extreme levels of relative risk aversion) for 26-asset portfolios and nine-asset portfolios. The highest mean across 
simulations of the proportionate opportunity cost found is $10.8 \%(0.108)$ for the level of relative risk aversion of 0.7 with extreme returns exaggerated. The lowest mean of the proportionate opportunity cost found is $0.4 \%(0.004)$ for the level of relative risk aversion of 31 for the original historical asset returns for the nine-asset portfolio. For both data sets, for the original historical asset returns and for historical asset returns with extreme values exaggerated, as the level of relative risk aversion increases the proportionate opportunity cost decreases.

The only difference between estimates of the proportionate opportunity cost for these two data sets is the magnitude of the estimates. They are bigger for the data sets when extreme returns exaggerated. This can be explained by the fact that the presence of extreme returns in the probability distribution moves mean-variance portfolio optimization much further from optimal (the presence of extreme returns moves the returns distribution away from elliptical symmetry, under which mean-variance portfolio optimization would be optimal).

My findings are different from those of Simaan (1993) and Tew, Reid and Witt (1991) in several ways. First, they differ in the magnitude of the opportunity cost: the highest opportunity cost Simaan found is $30 \%$ for the case with no riskless asset and $0.5 \%$ for the case with the riskless asset; the highest mean opportunity cost I found (with the nominally riskless asset included) is $5.6 \%$ for the 26 -asset portfolio and $3.5 \%$ for the nine-asset portfolio. Second, Simaan found that when the market offers a riskless asset, no matter what the level of risk aversion is the opportunity cost is almost zero. For the case with no riskless asset Simaan's and Tew, Reid and Witt's opportunity cost increases as risk aversion increases. I have worked with a semi-riskless asset: in nominal terms 
returns on Treasury bills are riskless, but in real terms there is no riskless asset (inflation is uncertain in any period and, thus, so are real returns on Treasury bills). So, with a semi-riskless asset I found that the opportunity cost decreases as risk aversion increases and it becomes almost zero for relative risk aversion greater than 29 for nine-asset portfolios. The differences in magnitude that I found between my results and Simaan' are due to several factors. I have used utility functions with constant relative risk aversion preferences, not with constant absolute risk aversion preferences. I also used a wide variety of levels of relative risk aversion (from 0.7 to 31 ), thus getting a range of values of the opportunity cost. Simaan on the other hand used levels of relative risk aversion of 2 and greater arguing that levels of risk aversion less than 2 offer very aggressive infeasible investment strategies.

Tew, Reid and Witt (1991) worked only with portfolios of risky assets (with the number of assets increasing from two to nine) and relative risk aversion that ranged from 0.1 to 1.9 (along with CRRA utility functions). They found very small opportunity costs for all considered levels of risk aversion. Also they found that as risk aversion increases the opportunity cost increases too. Their findings are consistent with Simaan's case where there was no riskless asset introduced. They also found that as the number of assets in portfolio increases the opportunity cost decreases. Tew, Reid and Witt's results are not consistent with mine, because even though I worked with 26 -asset portfolios and nineasset portfolios I also included a semi-riskless asset in both portfolios, which Tew, Reid and Witt did not do.

Therefore, based on my calculations, I may conclude that for investors with very high levels of relative risk aversion (29 and above) mean-variance analysis performs very 
well (with a relatively small number of assets in ones' portfolio) or significantly better (with a large number of assets in one's portfolio) than for investors with medium or low levels of relative risk aversion. So, as risk aversion increases mean-variance strategies show a fairly good approximation to the optimal portfolio strategy. 


\section{References:}

Campbell, John Y., Viceira, Luis H., Strategic Asset Allocation, Oxford University Press, March, 2002

Merton, Robert C. An analytic Derivation of the Efficient Portfolio Frontier, Journal of Financial and Quantitative Analysis, September 1972, 1851-72

Simaan, Yusif, What Is the Opportunity Cost of Mean-Variance Investment Strategy? Management Science, 1993, 39(5), 578-87

Tew, B.V., Reid, D.W., Witt, C.A., The Opportunity Cost of a Mean-Variance Efficient Choice, The Financial Review, 1991, 26(1), 31-43 


\title{
Chapter 3. Second Essay:
}

\section{The Opportunity Cost for an Investor of Being Constrained by}

\author{
the Number of Assets in His Portfolio
}

\begin{abstract}
Restricting the number of assets in an investor's portfolio results in a welfare loss for the investor. To measure that welfare loss I compare $n$-asset optimal portfolios with 26 -asset optimal portfolios by using the concept of the proportionate opportunity cost along with various CRRA utility functions. Two sets of asset returns are used with a VAR in generating joint returns distributions for the portfolio formation period: the original historical asset returns data set, and the historical asset returns with extreme values exaggerated. In each case 1,000 alternative sets of assets including one with a risk-free nominal return are randomly made available for investment. The analysis is performed with and without a short-selling constraint. I show that without the short-selling constraint and with no exaggeration of extreme values of asset returns the welldiversified number of assets is 24 . The number decreases as the level of risk aversion increases, and when the short-selling constraint is introduced. When the extreme values of asset returns are exaggerated in the asset returns distribution the well-diversified number of assets increases.

The second part of the essay explores investors' welfare losses when they restrict themselves to invest in either stocks or bonds but not both. The restriction gives investors suboptimal asset allocations that result in welfare losses for the investors. To measure those welfare losses I compare "only stock indices and Treasury bills" optimal portfolios or "only bond indices and Treasury bills" optimal portfolios with "stock and bond indices and Treasury bills" optimal portfolios, again using the concept of the proportionate opportunity cost along with various CRRA utility functions. The original historical asset returns data set is used with a VAR in generating joint returns distributions for the portfolio formation period. I show that for investors with low levels of risk aversion welfare losses do not exceed $1.5 \%$ of initial wealth when they invest sub-optimally. For investors with medium and high levels of relative risk aversion, constrained portfolios that include only one type of assets, stocks only or bonds only, along with Treasury bills, give expected utility about as high as unconstrained portfolios that include both types of assets, stocks and bonds.
\end{abstract}




\section{Chapter 3. Second Essay:}

\section{The Opportunity Cost for an Investor of Being Constrained by the Number of Assets in His Portfolio}

\section{Introduction}

The key question in the literature regarding well-diversified portfolios and the number of assets in portfolios is: at what point it is no longer very helpful to make more assets available for the portfolio? In other words, how can an investor tell whether his portfolio is well-diversified? Is there a substantial difference between diversification with the number of assets in an investor's portfolio constrained to be less than optimal investment strategy suggests (i.e. sub-optimally diversified portfolio) and optimal diversification?

Cheng and Liang (2000) address the last of those questions and found that there is evidence to support the idea that optimally diversified portfolios are more efficient than sub-optimally diversified portfolios in the context of mean-variance framework. To test the efficiency difference they set up and test the hypothesis that the Sharpe ratio for an efficient portfolio equals the Sharpe ratio of a sub-optimally diversified portfolio. The question that is left unanswered in the article and the question that I am interested in is: how inefficient is a sub-optimally diversified portfolio relative to an optimally diversified one?

In order to answer the question I will compare expected utility from the optimal portfolio constrained to include $n$ assets with that from the optimal unconstrained portfolio permitted to have 26 assets, as an approximation of an infinite number of assets 
that gives the highest diversification gain, by using the concept of opportunity cost. Then I will show how this comparison varies with $n$.

At a certain $n$ I will find that further diversification is no longer very helpful: the opportunity cost of investing in these $n$ assets rather than in 26 assets does not exceed $1 \%$ of initial wealth. Under the condition stated above the $n$ will be defined as a welldiversified number of assets.

The introduction to this dissertation showed that the proportionate opportunity cost is the best way to measure investors' welfare losses. Under the assumption of the constant relative risk aversion utility function

$$
\mathrm{U}(\widetilde{w})=\left\{\begin{array}{l}
\frac{1}{\gamma} \widetilde{w}^{\gamma}, \gamma<1, \gamma \neq 0, \widetilde{w}>0 \\
-\infty, \widetilde{w} \leq 0
\end{array}\right.
$$

the proportionate opportunity cost (willingness to accept payment as compensation for being constrained to only $n$ assets) can be calculated as $\theta-1.0$ where $\theta$ is defined by

$$
\mathrm{EU}\left(\theta w_{0} \widetilde{R}_{n}^{\text {optimal }}\right)=\mathrm{EU}\left(w_{0} \widetilde{R}_{26}^{\text {optimal }}\right)
$$

where $w_{0}$ is the initial wealth, $\widetilde{R}_{26}^{\text {optimal }}$ and $\widetilde{R}_{n}^{\text {optimal }}$ are the stochastic returns per dollar invested for the portfolios with 26 and $n$ assets respectively. Solving (2) with the utility function (1) gives

$$
\theta=\left[\frac{E\left(\widetilde{R}_{26}^{\gamma}\right)^{\text {optimal }}}{E\left(\widetilde{R}_{n}^{\gamma}\right)^{\text {optimal }}}\right]^{\frac{1}{\gamma}}
$$

Under CRRA $\theta$ also equals the ratio of certainty equivalents of the 26-asset unconstrained and $n$-asset constrained optimal portfolios, as shown in the introduction of this dissertation. Since the ratio of certainty equivalents is unitless, and in particular has 
no time units, the proportionate opportunity cost, $\theta-1.0$, is also timeless. But its numerical value depends on a number of months until horizon, i.e. with the investment horizon of $T$ months the proportionate willingness to accept payment to accept the constraint is $\theta^{T}$.

One motivation for finding the cost of sub-optimal diversification comes from Kelly (1995) which showed that “...[t]hree quarters of the households in the top quintile (of the survey sample) of stock ownership had fewer than ten different stocks". It raises the possibility that U.S. household behavior may not be well-diversified in terms of reducing idiosyncratic risk. The question arises: what is their cost of not diversifying well?

Brennan and Torous (1999) have addressed the issue of the cost of sub-optimal diversification, and Fama (1972) and Sankaran and Patil (1999) have addressed the issue of how many securities is enough for a well-diversified portfolio.

Brennan and Torous worked with a constant relative risk aversion utility function and with the certainty equivalent concept. They used the certainty equivalent as a way to measure the investor's loss when he diversifies sub-optimally. The authors randomly picked starting years and the securities for portfolios from CRSP. To form a portfolio they used the equally-weighted-portfolio rule (equal number of dollars invested in every asset). They formed portfolios with different numbers of assets in them. Then they calculated expected utility for those portfolios using a constant relative risk aversion utility function. The whole process of choosing a starting year, drawing securities, forming portfolios and calculating expected utility was repeated 10,000 times. Then, for every portfolio the certainty equivalent was calculated. And this certainty equivalent showed how much an investor would lose if he diversified sub-optimally (should he not 
have enough assets in his portfolio, not enough to call his portfolio well-diversified). The authors found that there are still significant welfare gains for an investor to be received even when the number of securities in the investor's portfolio is as high as 20 .

Brennan and Torous's equal-weighting rule of constructing portfolios is not appealing. The $\frac{1}{n}$ rule is characterized in the literature as a "naïve" portfolio strategy (Kroll et al. (1984)). The more appealing approach, which I will follow here, will be to use optimal portfolios that can be found through an optimization procedure. So, the comparison I am going to use will not be "equally-weighted-portfolio for $n$ assets versus equally-weighted-portfolio for 26 assets" but rather "optimal portfolio with $n$ assets versus optimal portfolio with 26 assets". Furthermore, my approach differs from theirs in terms of generating the ex ante returns distribution for the investment period. I use a vector autoregressive process (VAR) to project the means of returns and to capture 120 historically occurring shocks to all asset returns; then I assume that the true distribution of shocks for the investment period is given by adding those 120 sets of returns shocks with equal probabilities to the conditional means.

Working with the mean-variance theory Fama (1972) looked at the relationship between the standard deviation of a portfolio return and the number of assets in the portfolio. What he found is “...[m]ost of the effects of diversification ... occur when the first few securities are added to the portfolio. Once the portfolio has 20 securities, further diversification has little effect". To construct his portfolios Fama used randomly selected stocks and in his framework the cost of not investing in the optimal number of securities is measured in terms of the standard deviation of the portfolio return. He found that as the 
number of stocks in portfolios increases, the standard deviation of portfolio return decreases.

In his approach Fama (1972), like Brennan and Torous (1999), used the $\frac{1}{n}$ rule to construct all portfolios in order to do all his calculations and comparisons. And as was mentioned before, the existing literature characterizes the rule as a "naïve" portfolio strategy (Kroll et al. (1984)). And the rule is not appealing. The more appealing approach, which I will follow here, is to use optimal portfolios (not mean-variance efficient as Fama did, but rather globally optimal) that can be found through an optimization procedure.

Sankaran and Patil (1999), working with the same mean-variance theory, found that: “...[d]iversification beyond 8-10 securities may not be worthwhile". But this specific number of securities, as the authors pointed out, comes from optimally selecting a security to be constrained to zero quantity rather than randomly choosing a security to be excluded from the portfolio. In any event, their conclusion is: as the number of stocks in one's portfolio increases that will significantly reduce the risk of underperforming inflation and the stock market, and of losing portfolio value (Vassal (2001)).

The procedure used in the present essay, for calculating the proportionate opportunity cost for an investor of being constrained by the number of assets in his portfolio, includes random asset selection for investors' portfolios, estimation of a vector autoregressive process, derivation of the joint probability distribution function of asset returns, and computing optimal portfolios.

In the first part of the essay I show that with a nominally risk-free asset, the welldiversified number of assets in one's portfolio depends on degree of risk aversion, 
presence or absence of a short-selling constraint, and on the way a VAR process was used in deriving the asset returns distribution functions for the purpose of evaluating the opportunity cost. I found that the largest well-diversified number of assets in one's portfolio is 24 with no exaggeration of the extreme values of asset returns. When the extreme values of asset returns are exaggerated the largest well-diversified number of assets is 25 . As relative risk aversion increases the well-diversified number of assets in one's portfolio decreases. It is a very counterintuitive conclusion but a clear reason emerges. The well-diversified number of assets also decreases when a short-selling constraint is introduced. The results also show that for investors with high levels of risk aversion the well-diversified number of assets is less then three due to the fact that they place more than $90 \%$ of their initial wealth into Treasury bills. In the case with the unrestricted VAR the well-diversified number of assets is larger than that for the restricted VAR.

The second section of the essay describes the procedure of random asset selection for investors' portfolios, of inferring the joint probability distribution function of asset returns via a vector autoregression, of computing the constrained optimal and unconstrained optimal portfolios, and of the calculation of the proportionate opportunity cost; and it defines the short-selling constraint I use for some of the calculations. The third section discusses the results for the well-diversified number of assets.

The fourth section of the essay describes another type of a constrained portfolio strategy that is related to the well-diversified number of assets in one's portfolio: being constrained by investing in only stocks or only bonds, but not both. It uses the same procedures to find the cost of limiting oneself to investing in only stocks or only bonds, 
but not both. The section also concludes and summarizes the results for the "only stocks" and "only bonds" constraints.

\section{The Procedure}

\subsection{Asset Selection}

The procedure of calculating the proportionate opportunity cost for various $n$ for each of various levels of risk aversion will be performed 1,000 times, in each case using randomly picked nominally risky assets and Treasury bills as the nominally risk-free asset. The entire procedure will be performed with and without a short-selling constraint.

I compute opportunity costs for each degree of risk aversion with and without a short-selling constrained in a sequence of rounds $1,2,3, \ldots$ corresponding to $n=3,4,5$, ... . I start the first round by picking at random 25 nominally risky assets. The unconstrained optimal portfolio, with 26 assets, will include all 25 nominally risky assets and Treasury bills as the nominally risk-free asset. The constrained optimal portfolio, with $n=3$ assets, will include two nominally risky assets that I will pick at random from the 25 originally randomly picked nominally risky assets, and Treasury bills. Then, to construct the optimal constrained portfolio and optimal unconstrained portfolio I need to get expected values of real returns for time $T+1$ for all nominally risky assets and for nominally risk-free Treasury bills. In real terms, though, there is no risk-free asset. Returns on Treasury bills are risk-free only in nominal terms. But in time-series data inflation will be uncertain in any period and, thus, so will the real rate of return on Treasury bills. Therefore, the 26 assets and the three assets that I am dealing with will all be risky assets in real terms. 


\subsection{Vector Autoregressions of Returns}

So, to get expected values of real returns for the case of 26 assets and three assets at time $T+1$, the portfolio formation period, I estimate a vector autoregressive process (VAR). The next steps are to derive the joint probability distribution for the two groups of assets' real returns, and, finally, to construct optimal constrained and optimal unconstrained portfolios.

To derive the joint probability distribution of empirical deviations from the VARestimated conditional means for those 25 randomly picked asset returns and inflation I do the following.

The nominal return on asset $i$ at time $t$ minus the nominal return on Treasury bills at time $t$ gives us the excess return on asset $i\left(x_{i, t}\right)$ at time $t$ for $i=1, \ldots, 25$ and for $t=1, \ldots, T$. When I run a VAR for excess returns of those 25 assets and realized inflation, as

$$
\left[\begin{array}{c}
x_{1, t} \\
\cdot \\
x_{25, t} \\
\pi_{t}
\end{array}\right]=\left[\begin{array}{c}
c_{1} \\
\cdot \\
c_{25} \\
c_{26}
\end{array}\right]+\left[\begin{array}{cccc}
v_{1,1}(L) & \cdot & \cdot & v_{1,26}(L) \\
\cdot & \cdot & \cdot & \cdot \\
v_{25,1}(L) & \cdot & \cdot & v_{25,26}(L) \\
v_{26,1}(L) & \cdot & \cdot & v_{26,26}(L)
\end{array}\right]\left[\begin{array}{c}
x_{1, t} \\
\cdot \\
x_{25, t} \\
\pi_{t}
\end{array}\right]+\left[\begin{array}{c}
\varepsilon_{1, t} \\
\cdot \\
\varepsilon_{25, t} \\
\varepsilon_{\pi, t}
\end{array}\right],
$$

I obtain $\left\{\hat{c}_{i}\right\},\left\{\hat{\varepsilon}_{i, t}\right\}$ and $\left\{\hat{v}_{i, k}(L)\right\}$, where

$$
\hat{v}_{i, k}(L)=\hat{\delta}_{i, k}^{1} L^{1}+\hat{\delta}_{i, k}^{2} L^{2}+\ldots
$$

Then, I compute the vector of conditional expected values of excess returns for time $T+1$ and expected inflation for time $T+1$ as:

$$
\left[\begin{array}{c}
E_{T} x_{1, T+1} \\
\cdot \\
E_{T} x_{25, T+1} \\
E_{T} \pi_{T+1}
\end{array}\right]=\left[\begin{array}{c}
\hat{c}_{1} \\
\cdot \\
\hat{c}_{25} \\
\hat{c}_{26}
\end{array}\right]+\left[\begin{array}{cccc}
\hat{v}_{1,1}(L) & \cdot & \cdot & \hat{v}_{1,26}(L) \\
\cdot & \cdot & \cdot & \cdot \\
\hat{v}_{25,1}(L) & \cdot & \cdot & \hat{v}_{25,26}(L) \\
\hat{v}_{26,1}(L) & \cdot & \cdot & \hat{v}_{26,26}(L)
\end{array}\right]\left[\begin{array}{c}
x_{1, T+1} \\
\cdot \\
x_{25, T+1} \\
\pi_{T+1}
\end{array}\right] .
$$

Next, the expected real return on asset $i$ in period $T+1$, the portfolio formation period, is 


$$
\left[\begin{array}{c}
E_{T} r_{1, T+1} \\
\cdot \\
E_{T} r_{25, T+1}
\end{array}\right]=\left[\begin{array}{c}
E_{T} x_{1, T+1} \\
\cdot \\
E_{T} x_{25, T+1}
\end{array}\right]+\left[\begin{array}{c}
r_{T B, T+1}^{n} \\
\cdot \\
r_{T B, T+1}^{n}
\end{array}\right]-\left[\begin{array}{c}
E_{T} \pi_{T+1} \\
\cdot \\
E_{T} \pi_{T+1}
\end{array}\right]
$$

where $r_{T B, T+1}^{n}$ is the ex ante observed nominal return on Treasury bills for time $T+1$. The expected real return on Treasury bills for time $T+1$ is

$$
E_{T} r_{T B, T+1}=r_{T B, T+1}^{n}-E_{T} \pi_{T+1} .
$$

Finally, the conditional probability distribution for real returns for time $T+1$ is determined by

$$
\left[\begin{array}{c}
\widetilde{r}_{1, T+1} \\
\cdot \\
\widetilde{r}_{25, T+1} \\
\widetilde{r}_{T B, T+1}
\end{array}\right]=\left[\begin{array}{c}
E_{T} x_{1, T+1} \\
\cdot \\
E_{T} x_{25, T+1} \\
0
\end{array}\right]+\left[\begin{array}{c}
r_{T B, T+1}^{n} \\
\cdot \\
r_{T B, T+1}^{n} \\
r_{T B, T+1}^{n}
\end{array}\right]-\left[\begin{array}{c}
E_{T} \pi_{T+1} \\
\cdot \\
E_{T} \pi_{T+1} \\
E_{T} \pi_{T+1}
\end{array}\right]+\left[\begin{array}{c}
\widetilde{\varepsilon}_{1, T+1} \\
\cdot \\
\widetilde{\varepsilon}_{25, T+1} \\
0
\end{array}\right]-\left[\begin{array}{c}
\widetilde{\varepsilon}_{\pi, T+1} \\
\cdot \\
\widetilde{\varepsilon}_{\pi, T+1} \\
\widetilde{\varepsilon}_{\pi, T+1}
\end{array}\right]
$$

where $\left[\begin{array}{c}\widetilde{\varepsilon}_{1, T+1} \\ \cdot \\ \widetilde{\varepsilon}_{25, T+1} \\ \widetilde{\varepsilon}_{\pi, T+1}\end{array}\right]$ takes on the historically observed values $\left[\begin{array}{c}\widetilde{\varepsilon}_{1, t} \\ \cdot \\ \widetilde{\varepsilon}_{25, t} \\ \widetilde{\varepsilon}_{\pi, t}\end{array}\right]$ from regression (4), $t=1,2, \ldots, T$, with equal probabilities $(1 / T)$.

This way of deriving asset returns probability distribution functions, using historically occurring innovations to asset returns captured through this VAR procedure, is superior to the VAR method mentioned in the literature, e.g. Campbell and Viceira (2002). The literature on derivation of asset returns probability distribution functions assumes that the distribution of asset returns is static, not evolving over time. But the reality is such that the asset returns distribution is dynamic, depending on both recent realizations and the fixed historical distribution of shocks to the dynamic asset returns 
process. So the right way of deriving asset returns probability distribution functions is to include the dynamics of the past history of asset returns.

Similarly, to get the probability distribution of returns for use in three-asset portfolios, the above procedure including the VAR is redone using (4)-(9) with 26 changed to three.

\subsection{Constrained Portfolios}

Using the information about those three assets' derived probability distribution for their real returns (computed using the equations analogous to (4)-(9)), I compute the constrained optimal portfolio with $n=3$ assets: the solution of

$$
\underset{\left\{\alpha_{1}, \alpha_{2}\right\}}{\operatorname{Max}} E U(\widetilde{w})=\operatorname{Max} E\left\{\frac{1}{\gamma}\left[w_{0}\left(\alpha_{1} \widetilde{r}_{1}+\alpha_{2} \widetilde{r}_{2}+\left(1-\alpha_{1}-\alpha_{2}\right) \widetilde{r}_{T B}\right)\right]^{\gamma}\right\}
$$

where $\alpha_{1}, \alpha_{2}, 1-\alpha_{1}-\alpha_{2}$ are the three individual assets' portfolio shares in the constrained optimal portfolio. To get the portfolio I search over $\alpha_{1}, \alpha_{2}$ space to optimize expected utility, using nonlinear optimization by a quasi-Newton method based on convergence to first-order conditions of problem (10). The expectation is taken over the joint probability distribution derived as described above analogously to (4)-(9).

\subsection{Unconstrained portfolios}

The next step, then, is to get the unconstrained optimal portfolio with 26 assets: the solution of

$$
\underset{\left\{\alpha_{1}, \ldots, \alpha_{25}\right\}}{\operatorname{Max}} E U(\widetilde{w})=\operatorname{Max} E\left\{\frac{1}{\gamma}\left[w_{0}\left(\alpha_{1} \widetilde{r}_{1}+\ldots+\alpha_{25} \widetilde{r}_{25}+\left(1-\alpha_{1}-\ldots-\alpha_{25}\right) \widetilde{r}_{T B}\right)\right]^{\gamma}\right\}
$$


where $\alpha_{1}, \ldots, \alpha_{25}$ are the first 25 individual assets' portfolio shares in the unconstrained optimal portfolio. To get the portfolio I search over $\alpha_{1}, \ldots, \alpha_{25}$ space to optimize expected utility, again using nonlinear optimization by a quasi-Newton method based on convergence to first-order conditions of problem (11). This time, the expectation is taken over the joint probability distribution derived as described above in (4)-(9).

\subsection{Calculating Opportunity Cost}

Now, when I have the constrained and unconstrained optimal portfolios I calculate the opportunity cost, $\theta-1.0$. For the formula for $\theta$, (3), I need to find $\mathrm{E}\left(\widetilde{R}_{26}^{\gamma}\right)^{\text {optimal }}$, where $\widetilde{R}_{26}$ is the gross return for the optimal unconstrained portfolio with 26 assets, and $\mathrm{E}\left(\widetilde{R}_{n}^{\gamma}\right)^{\text {optimal }}$, where $\widetilde{R}_{n}$ is the gross return for the constrained optimal portfolio with three assets.

$$
\mathrm{E}\left(\widetilde{R}_{26}^{\gamma}\right)^{\text {optimal }} \text { (referring more completely to } E_{T}\left(\widetilde{R}_{26, T+1}^{\gamma}\right)^{\text {optimal }} \text { ) is equal to }
$$

$$
E_{T}\left(\widetilde{R}_{26, T+1}^{\gamma}\right)^{\text {optimal }}=\frac{1}{T} \sum_{t=1}^{T}\left\{\left[\begin{array}{llll}
\alpha_{1}^{* *} & \ldots & \alpha_{25}^{* *} & 1-\alpha_{1}^{* *}-\ldots-\alpha_{25}^{* *}
\end{array}\right]\left[\begin{array}{c}
E_{T} r_{1, T+1}+\varepsilon_{1, t}-\varepsilon_{\pi, t} \\
\cdot \\
E_{T} r_{25, T+1}+\varepsilon_{25, t}-\varepsilon_{\pi, t} \\
E_{T} r_{T B, T+1}+0-\varepsilon_{\pi, t}
\end{array}\right]\right\}^{\gamma}
$$

where the vector of $\alpha_{i}^{* *}$ is the vector of optimal portfolio shares for the portfolio with 26 assets; the vectors of $E_{T} r_{i, T+1}+\varepsilon_{i, t}-\varepsilon_{\pi, t}$ and $E_{T} r_{T B, T+1}-\varepsilon_{\pi, \mathrm{t}}$ are the vectors of particular possible values of real returns (conditional on the data set for times $t=1$ through $T$ ) at time $T+1$ (the portfolio formation period) and calculated as shown in (4)-(9).

$$
\text { And } \mathrm{E}\left(\widetilde{R}_{n}^{\gamma}\right)^{\text {optimal }} \text { is equal to }
$$




$$
E_{T}\left(\widetilde{R}_{3, T+1}^{\gamma}\right)^{\text {optimal }}=\frac{1}{T} \sum_{t=1}^{T}\left\{\left[\begin{array}{lll}
\alpha_{1}^{*} & \alpha_{2}^{*} & 1-\alpha_{1}^{*}-\alpha_{2}^{*}
\end{array}\right]\left[\begin{array}{c}
E_{T} r_{1, T+1}+\varepsilon_{1, t}-\varepsilon_{\pi, t} \\
E_{T} r_{2, T+1}+\varepsilon_{2, t}-\varepsilon_{\pi, t} \\
E_{T} r_{T B, T+1}+0-\varepsilon_{\pi, t}
\end{array}\right]\right\}^{\gamma}
$$

where $\alpha_{i}^{*}$ is the vector of optimal portfolio shares for the portfolio with $n$ assets. In (13) the expectations are taken over the distribution implied by the $n$-asset (in this case 3asset) VAR. Thus the $\theta$ calculations will reflect only the cost of restricting the number of assets. Subsequently the $\theta$ calculations will be redone, taking the expectations in (13) over the distribution implied by the 26 -asset VAR. These $\theta$ 's will also reflect the cost of having chosen the $n$-asset portfolios using a restricted size of the VAR.

Then, having calculated (12) and (13), I use (3) to get a numerical value for $\theta$. And the proportionate opportunity cost for an investor of investing in three assets rather than in 26 assets is simply $\theta-1.0$.

I start the second round by retaining the 25 originally picked nominally risky assets and Treasury bills. This time, again, the 26-asset portfolio will be the unconstrained optimal portfolio, and $\mathrm{E}\left(\widetilde{R}_{26}^{\gamma}\right)^{\text {optimal }}$ is equal to $\mathrm{E}\left(\widetilde{R}_{26, T+1}^{\gamma}\right)^{\text {optimal }}$ as already computed. The constrained optimal portfolio this time will include four assets including Treasury bills, and to get that I pick three nominally risky assets at random from the original 25. Then, I get expected values of real returns for the four assets including Treasury bills for time $T+1$ by estimating a new four-variable vector autoregressive process. After that I derive the joint probability distribution for the four assets' real returns, and construct the optimal constrained four-asset portfolio. Then, $\mathrm{E}\left(\widetilde{R}_{n}^{\gamma}\right)^{\text {optimal }}$ is 
equal to $\mathrm{E}\left(\widetilde{R}_{4, T+1}^{\gamma}\right)^{\text {optimal }}$. Finally, using (13) with $n$ updated I calculate $\theta$ and hence the proportionate opportunity cost of investing in four assets rather than in 26 assets.

For each round, the procedure is repeated 1,000 times. This gives me 1,000 values of $\theta$ for each $n$. I do this (round after round) until the mean value of the proportionate opportunity cost of investing in $n$ assets rather than in 26 is no larger than $1 \%$. The entire procedure is done for each of 11 alternative values of the risk aversion parameter $\gamma$.

\subsection{Short-Selling Restriction}

Short-selling is a legitimate trading strategy. By short-selling an investor will get extra money to invest and will make positive returns if the shorted assets' price rises by less than that of the assets in which the extra funds are placed. At the same time it is a risky strategy because the investor will lose money if the price of the shorted asset rises by more than that of the assets in which the extra funds are placed. An optimal portfolio may require an investor to hold extremely long or extremely short investment positions. These extreme long or short holding positions sometimes are difficult to implement in practice because investors face constraints on their portfolio holdings. For example, Regulation $\mathrm{T}$ that applies to almost all investors, institutional as well as individual, requires $50 \%$ margin.

Any short-selling restriction will reduce investment opportunities for an investor. In this context it is interesting to see how these restrictions affect the well-diversified number of assets in the investor's portfolio.

The $50 \%$ margin restriction is implemented in the essay the following way. First I restrict short sales of each individual asset to be no more than $50 \%$ of initial wealth: 


$$
\alpha_{i} \geq-0.5 w_{0} \quad \forall i
$$

where $\alpha_{i}$ 's are individual portfolio shares, and $w_{0}$ is initial wealth set equal to 1 . Then I find an optimal portfolio and check if the sum of all negative-valued optimal portfolio shares is less than -0.5 . If it is not, then I proceed with the calculation of the proportionate opportunity cost. If the sum of all negative-valued individual portfolio shares is indeed less than -0.5 , then I change the restriction on short sales of each individual asset to the lower proportion $40 \%$ of initial wealth, and change (14) appropriately:

$$
\alpha_{i} \geq-0.4 w_{0} \quad \forall i
$$

Then, again, I find an optimal portfolio and check if the sum of all negative-valued optimal portfolio shares is less than -0.5 . If it is not, then I proceed with the calculation of the proportionate opportunity cost. But if the sum of all negative-valued individual portfolio shares is again less than -0.5 , then I restrict short sales of each individual asset to the even lower proportion $30 \%$ of initial wealth, and with the appropriate change to (14) I find an optimal portfolio.

These reductions of the proportion of initial wealth that allows short positions for individual assets will take place until either the sum of all negative-valued individual portfolio shares is less than -0.5 or the proportion of initial wealth allowed for short positions for individual assets reaches zero. In the latter case the short-selling restriction, that the sum of short sales of each individual asset must not exceed $50 \%$ of initial wealth, holds automatically. 


\section{Results}

The results from this research project are as follows.

\subsection{Results Derived for Opportunity Cost of Restricting the Number of Assets in Portfolios.}

This part of the section discusses the results derived from calculations of the proportionate opportunity costs that reflect only the cost of restricting the number of assets in investors' portfolios. In the course of calculating these costs, $n$-asset and 26asset VARs were used. Therefore, the expectations in (12) and (13) were taken over the distributions of asset returns implied by the two different VARs.

\subsubsection{Results Derived from Historical Returns Data with no Exaggeration of Extreme Returns}

\subsubsection{Opportunity Costs with and without a Short-Selling Constraint}

Table 1 and Table 2 present the results from calculation of 1,000 values of the proportionate opportunity cost for each of 11 different values of relative risk aversion for portfolios with three to 25 assets with no short-selling constraint, and with the shortselling constraint, based on returns distributions derived from historically occurring asset returns over the 10-year period January 1992 through December 2001.

For relative risk aversion of 0.7 for the case with no short-selling constraint the number of assets beyond which the mean value (over 1,000 replications) of the opportunity cost is no higher than $1 \%$ of initial wealth is 24 . With 24 assets in the investor's portfolio for the risk aversion level of 0.7 it is no longer helpful to make more assets available for the portfolio: Table 1 reports that the mean value (over 1,000 


\section{Table 1}

The proportionate opportunity cost of restricting the number of assets in portfolios, $(\theta-1)$, for various values of relative risk aversion with no short-selling constraint (mean values over 1,000 replications)

\begin{tabular}{|c|c|c|c|c|c|c|c|c|c|c|c|}
\hline $\begin{array}{c}\text { Number of } \\
\text { assets } \\
\text { in portfolios }\end{array}$ & 0.7 & 1 & 2 & 3 & 9 & 10 & 11 & 12 & 29 & 30 & 31 \\
\hline 3 & 0.165 & 0.118 & 0.062 & 0.041 & 0.013 & 0.012 & 0.012 & 0.011 & $\mathbf{0 . 0 0 6}$ & $\mathbf{0 . 0 0 5}$ & $\mathbf{0 . 0 0 4}$ \\
4 & 0.159 & 0.092 & 0.061 & 0.039 & 0.012 & 0.012 & 0.011 & 0.011 & 0.005 & 0.003 & 0.002 \\
5 & 0.157 & 0.091 & 0.058 & 0.038 & 0.012 & 0.011 & 0.011 & 0.011 & 0.005 & 0.003 & 0.001 \\
6 & 0.147 & 0.084 & 0.056 & 0.036 & 0.011 & 0.011 & 0.011 & $\mathbf{0 . 0 1 0}$ & 0.003 & 0.001 & 0.001 \\
7 & 0.141 & 0.081 & 0.053 & 0.034 & 0.011 & 0.011 & $\mathbf{0 . 0 1 0}$ & 0.007 & 0.003 & 0.001 & 0.000 \\
8 & 0.135 & 0.080 & 0.051 & 0.032 & 0.011 & $\mathbf{0 . 0 1 0}$ & 0.008 & 0.007 & 0.001 & 0.000 & 0.000 \\
9 & 0.126 & 0.078 & 0.049 & 0.030 & $\mathbf{0 . 0 1 0}$ & 0.009 & 0.007 & 0.006 & 0.001 & 0.000 & 0.000 \\
10 & 0.119 & 0.077 & 0.045 & 0.029 & 0.009 & 0.008 & 0.007 & 0.005 & 0.000 & 0.000 & 0.000 \\
11 & 0.113 & 0.075 & 0.042 & 0.027 & 0.008 & 0.007 & 0.005 & 0.005 & 0.000 & 0.000 & 0.000 \\
12 & 0.105 & 0.073 & 0.040 & 0.026 & 0.007 & 0.007 & 0.005 & 0.005 & 0.000 & 0.000 & 0.000 \\
13 & 0.098 & 0.069 & 0.037 & 0.024 & 0.007 & 0.006 & 0.005 & 0.003 & 0.000 & 0.000 & 0.000 \\
14 & 0.090 & 0.063 & 0.034 & 0.023 & 0.006 & 0.005 & 0.003 & 0.001 & 0.000 & 0.000 & 0.000 \\
15 & 0.083 & 0.057 & 0.031 & 0.020 & 0.006 & 0.005 & 0.003 & 0.001 & 0.000 & 0.000 & 0.000 \\
16 & 0.076 & 0.054 & 0.029 & 0.019 & 0.006 & 0.005 & 0.003 & 0.001 & 0.000 & 0.000 & 0.000 \\
17 & 0.071 & 0.051 & 0.027 & 0.017 & 0.005 & 0.005 & 0.001 & 0.000 & 0.000 & 0.000 & 0.000 \\
18 & 0.063 & 0.044 & 0.024 & 0.015 & 0.005 & 0.004 & 0.001 & 0.000 & 0.000 & 0.000 & 0.000 \\
19 & 0.054 & 0.040 & 0.021 & 0.011 & 0.005 & 0.003 & 0.000 & 0.000 & 0.000 & 0.000 & 0.000 \\
20 & 0.047 & 0.035 & 0.018 & $\mathbf{0 . 0 1 0}$ & 0.004 & 0.003 & 0.000 & 0.000 & 0.000 & 0.000 & 0.000 \\
21 & 0.037 & 0.028 & 0.012 & 0.007 & 0.003 & 0.000 & 0.000 & 0.000 & 0.000 & 0.000 & 0.000 \\
22 & 0.028 & 0.018 & $\mathbf{0 . 0 1 0}$ & 0.005 & 0.000 & 0.000 & 0.000 & 0.000 & 0.000 & 0.000 & 0.000 \\
23 & 0.019 & $\mathbf{0 . 0 1 0}$ & 0.006 & 0.003 & 0.000 & 0.000 & 0.000 & 0.000 & 0.000 & 0.000 & 0.000 \\
24 & $\mathbf{0 . 0 1 0}$ & 0.006 & 0.003 & 0.000 & 0.000 & 0.000 & 0.000 & 0.000 & 0.000 & 0.000 & 0.000 \\
25 & 0.004 & 0.030 & 0.000 & 0.000 & 0.000 & 0.000 & 0.000 & 0.000 & 0.000 & 0.000 & 0.000 \\
\hline
\end{tabular}

replications) of the proportionate opportunity cost of investing in 25 assets rather than in 26 is $0.4 \%(0.004)$. As risk aversion increases, though, the well-diversified number of assets in investors' portfolio decreases. It reaches 20 assets for risk aversion of three; nine assets for risk aversion of nine; and three or less assets for investors with risk aversion of 29 and higher.

For the case with no short-selling constraint, as Table 1 shows, the highest mean (over 1,000 replications) of the proportionate opportunity cost, 16.5\%, corresponds to the 


\section{Table 2}

The proportionate opportunity cost of restricting the number of assets in portfolios, $(\theta-1)$, for various values of relative risk aversion with the short-selling constraint (mean values over 1,000 replications)

\begin{tabular}{|c|c|c|c|c|c|c|c|c|c|c|c|}
\hline $\begin{array}{c}\text { Number of } \\
\text { assets } \\
\text { in portfolios }\end{array}$ & 0.7 & 1 & 2 & 3 & 9 & 10 & 11 & 12 & 29 & 30 & 31 \\
\hline 3 & 0.021 & 0.019 & 0.017 & 0.016 & 0.011 & 0.010 & $\mathbf{0 . 0 1 0}$ & $\mathbf{0 . 0 1 0}$ & $\mathbf{0 . 0 0 5}$ & $\mathbf{0 . 0 0 5}$ & $\mathbf{0 . 0 0 4}$ \\
4 & 0.019 & 0.018 & 0.016 & 0.014 & 0.010 & $\mathbf{0 . 0 1 0}$ & 0.009 & 0.008 & 0.003 & 0.003 & 0.002 \\
5 & 0.017 & 0.016 & 0.014 & 0.012 & $\mathbf{0 . 0 1 0}$ & 0.009 & 0.008 & 0.006 & 0.001 & 0.001 & 0.000 \\
6 & 0.016 & 0.015 & 0.011 & 0.011 & 0.009 & 0.007 & 0.006 & 0.004 & 0.000 & 0.000 & 0.000 \\
7 & 0.014 & 0.012 & 0.011 & 0.011 & 0.008 & 0.006 & 0.004 & 0.002 & 0.000 & 0.000 & 0.000 \\
8 & 0.013 & 0.011 & 0.010 & $\mathbf{0 . 0 1 0}$ & 0.006 & 0.004 & 0.002 & 0.001 & 0.000 & 0.000 & 0.000 \\
9 & 0.011 & 0.010 & $\mathbf{0 . 0 1 0}$ & 0.009 & 0.005 & 0.002 & 0.001 & 0.000 & 0.000 & 0.000 & 0.000 \\
10 & 0.010 & $\mathbf{0 . 0 1 0}$ & 0.009 & 0.008 & 0.004 & 0.001 & 0.000 & 0.000 & 0.000 & 0.000 & 0.000 \\
11 & $\mathbf{0 . 0 1 0}$ & 0.009 & 0.008 & 0.007 & 0.002 & 0.001 & 0.000 & 0.000 & 0.000 & 0.000 & 0.000 \\
12 & 0.009 & 0.008 & 0.007 & 0.006 & 0.001 & 0.000 & 0.000 & 0.000 & 0.000 & 0.000 & 0.000 \\
13 & 0.008 & 0.007 & 0.006 & 0.005 & 0.001 & 0.000 & 0.000 & 0.000 & 0.000 & 0.000 & 0.000 \\
14 & 0.007 & 0.006 & 0.005 & 0.005 & 0.000 & 0.000 & 0.000 & 0.000 & 0.000 & 0.000 & 0.000 \\
15 & 0.006 & 0.006 & 0.005 & 0.005 & 0.000 & 0.000 & 0.000 & 0.000 & 0.000 & 0.000 & 0.000 \\
16 & 0.005 & 0.005 & 0.005 & 0.004 & 0.000 & 0.000 & 0.000 & 0.000 & 0.000 & 0.000 & 0.000 \\
17 & 0.005 & 0.005 & 0.004 & 0.004 & 0.000 & 0.000 & 0.000 & 0.000 & 0.000 & 0.000 & 0.000 \\
18 & 0.005 & 0.004 & 0.003 & 0.003 & 0.000 & 0.000 & 0.000 & 0.000 & 0.000 & 0.000 & 0.000 \\
19 & 0.004 & 0.004 & 0.003 & 0.002 & 0.000 & 0.000 & 0.000 & 0.000 & 0.000 & 0.000 & 0.000 \\
20 & 0.004 & 0.003 & 0.002 & 0.001 & 0.000 & 0.000 & 0.000 & 0.000 & 0.000 & 0.000 & 0.000 \\
21 & 0.004 & 0.003 & 0.001 & 0.001 & 0.000 & 0.000 & 0.000 & 0.000 & 0.000 & 0.000 & 0.000 \\
22 & 0.004 & 0.003 & 0.001 & 0.001 & 0.000 & 0.000 & 0.000 & 0.000 & 0.000 & 0.000 & 0.000 \\
23 & 0.003 & 0.001 & 0.001 & 0.000 & 0.000 & 0.000 & 0.000 & 0.000 & 0.000 & 0.000 & 0.000 \\
24 & 0.003 & 0.001 & 0.000 & 0.000 & 0.000 & 0.000 & 0.000 & 0.000 & 0.000 & 0.000 & 0.000 \\
25 & 0.003 & 0.001 & 0.000 & 0.000 & 0.000 & 0.000 & 0.000 & 0.000 & 0.000 & 0.000 & 0.000 \\
\hline
\end{tabular}

lowest level of risk aversion of 0.7 and will be incurred by an investor should he decide to invest in three assets rather than in 26. The lowest mean (over 1,000 replications) of the proportionate opportunity cost, $0.0 \%$, corresponds to the highest level of risk aversion of 31 and will be incurred by an investor should he decide to invest in seven or more assets rather than in 26 .

The mean values (over 1,000 replications) of the proportionate opportunity cost of investing in four assets rather than 26 range from 15.9\% for the level of risk aversion of 
0.7 to $0.2 \%$ for the level of risk aversion of 31 . The mean values (over 1,000 replications) of the proportionate opportunity cost of investing in five assets rather than 26 range from $15.7 \%$ for the level of risk aversion of 0.7 to $0.1 \%$ for the level of risk aversion of 31 . The mean values (over 1,000 replications) of the proportionate opportunity cost of investing in ten assets rather than 26 range from $11.9 \%$ for the level of risk aversion of 0.7 to $0.0 \%$ for the level of risk aversion of 31 . It is clear that the mean values of the proportionate opportunity cost of investing in $n$ assets rather than in 26 with no shortselling constraint decreases as the number of assets available for investment increases, and as the level of relative risk aversion increases.

With the short-selling constraint, for risk aversion of 0.7 the number of assets beyond which the mean value (over 1,000 replications) of the proportionate opportunity cost is no higher than $1 \%$ of initial wealth is 11 . It reaches eight assets for risk aversion of three; five assets for risk aversion of nine; four assets of risk aversion of ten; and three or less assets for risk aversion of 11 and higher.

As Table 2 shows, for the case with the short-selling constraint, the highest mean (over 1,000 replications) of the proportionate opportunity cost, $2.1 \%$, corresponds to the lowest level of risk aversion of 0.7 and will be incurred by an investor should he decide to invest in three assets rather than in 26 . The lowest mean (over 1,000 replications) of the proportionate opportunity cost, $0.0 \%$, corresponds to the highest level of risk aversion of 31 and will be incurred by an investor should he decide to invest in five or more assets rather than in 26.

The mean values (over 1,000 replications) of the proportionate opportunity cost of investing in four assets rather than 26 range from $1.9 \%$ for the level of risk aversion of 
0.7 to $0.2 \%$ for the level of risk aversion of 31 . The mean values (over 1,000 replications) of the proportionate opportunity cost of investing in five assets rather than 26 range from $1.7 \%$ for the level of risk aversion of 0.7 to $0.0 \%$ for the level of risk aversion of 31 . The mean values (over 1,000 replications) of the proportionate opportunity cost of investing in ten assets rather than 26 range from $1.0 \%$ for the level of risk aversion of 0.7 to $0.0 \%$ for the level of risk aversion of 31 .

These results agree with the conclusion for the case with no short-selling constraint that the mean values of the proportionate opportunity cost of investing in $n$ assets rather than in 26 decreases as the number of assets available for investment increases. This makes sense. When one more asset becomes available for investment, this extra asset takes an investor's portfolio to a higher diversification level with lower than before unsystematic risk, and thus to a higher welfare level. But the investor's marginal benefit from adding one more new asset to his portfolio decreases with every new asset. This marginal investor's benefit decreases more and more as more and more assets become available for investment. That is why as the number of assets in investors' portfolios increases, the proportionate opportunity cost of not investing in 26 assets decreases.

The results from Table 2 also agree with another conclusion for the case with no short-selling constraint, that the mean value of the proportionate opportunity cost of investing in $n$ assets rather than in 26 decreases as the level of relative risk aversion increases. This also makes sense. As the level of risk aversion increases, as investors become more and more afraid of risk, they will prefer to increase the fraction of their initial wealth placed in the nominally risk-free asset, Treasury bills (see Table 3 ), and 
whatever is left will be divided between nominally risky assets. So, even if they increase the number of nominally risky assets in their portfolios, the diversification benefit will not be as substantial as for investors with a lower level of risk aversion due to the smaller fraction of the initial wealth placed in the nominally risky assets. Therefore, the mean values of the proportionate opportunity cost of investing in $n$ assets rather than in 26 for investors with higher levels of risk aversion will be lower.

The only difference between Table 1 and Table 2 is that with the short-selling constraint the optimal number of assets in investors' portfolios is lower than that for portfolios with no short-selling constraint. And this is the result of placing the constraint on investors' behavior. This constraint will reduce any diversification benefits. The answer to the question of how much a short-selling constraint reduces investors' welfare due to the reduction of the diversification benefits will be answered in Chapter 4 of this dissertation.

\subsubsection{Optimal portfolio shares}

Table 3 and Table 4 present typical optimal portfolio shares for unconstrained (26-asset) and constrained portfolio strategies for three different levels of relative risk aversion: low (of 0.7), medium (of 11) and high (of 31). Table 3 presents optimal portfolio shares with no short-selling constraint; Table 4 presents optimal portfolio shares with a short-selling constraint. For all three levels of risk aversion there is a different well-diversified number of assets; therefore, constrained optimal portfolios for different levels of risk aversion have different numbers of assets in them. In each case illustrative optimal portfolio shares are calculated for a different set of available assets whose 
Table 3

Illustrative optimal portfolio shares for unconstrained and optimally constrained to include $\boldsymbol{n}$ assets portfolio strategies for different values of relative risk aversion ${ }^{1}$

(with no short-selling constraint)

\begin{tabular}{|c|c|c|c|c|c|c|}
\hline \multirow[t]{2}{*}{ Asset \# } & \multicolumn{2}{|c|}{$\begin{array}{l}\text { Relative Risk Aversion, }(1-\gamma) \text {, } \\
\text { equal to } 0.7\end{array}$} & \multicolumn{2}{|c|}{$\begin{array}{c}\text { Relative Risk Aversion, } \\
(1-\gamma) \text {, equal to } 11\end{array}$} & \multicolumn{2}{|c|}{$\begin{array}{c}\text { Relative Risk Aversion, } \\
(1-\gamma) \text {, equal to } 31\end{array}$} \\
\hline & Unconstrained & Constrained & Unconstrained & Constrained & Unconstrained & Constrained \\
\hline 1 & 3.055 & 2.764 & 0.049 & 0.000 & 0.001 & 0.000 \\
\hline 2 & -0.081 & -0.129 & 0.024 & 0.006 & 0.049 & 0.023 \\
\hline 3 & 0.337 & 0.000 & 0.076 & 0.000 & -0.081 & 0.000 \\
\hline 4 & 2.621 & 2.145 & -1.000 & 0.000 & 0.016 & 0.000 \\
\hline 5 & -0.427 & -0.502 & 0.066 & 0.000 & 0.166 & 0.088 \\
\hline 6 & 0.149 & 0.008 & 0.195 & 0.000 & 0.001 & 0.000 \\
\hline 7 & 0.638 & 0.664 & 0.031 & 0.182 & 0.035 & 0.000 \\
\hline 8 & -0.019 & -0.385 & -0.026 & 0.000 & 0.049 & 0.000 \\
\hline 9 & -1.991 & -2.197 & -0.014 & 0.000 & 0.008 & 0.000 \\
\hline 10 & -0.305 & -0.309 & 0.049 & 0.000 & 0.018 & 0.000 \\
\hline 11 & 0.647 & 0.544 & -0.042 & 0.000 & 0.066 & 0.000 \\
\hline 12 & 1.004 & 1.191 & 0.097 & 0.089 & -0.029 & 0.000 \\
\hline 13 & 1.456 & 1.515 & 0.007 & 0.002 & 0.016 & 0.000 \\
\hline 14 & 0.119 & 0.095 & -0.082 & 0.000 & -0.007 & 0.000 \\
\hline 15 & -0.572 & -0.396 & 0.201 & 0.191 & -0.049 & 0.000 \\
\hline 16 & 0.137 & 0.289 & 0.053 & 0.000 & -0.082 & 0.000 \\
\hline 17 & -1.837 & -1.827 & 0.091 & 0.000 & 0.021 & 0.000 \\
\hline 18 & 0.001 & 0.172 & -0.025 & 0.000 & 0.005 & 0.000 \\
\hline 19 & 0.266 & 0.294 & 0.059 & 0.000 & 0.018 & 0.000 \\
\hline 20 & -0.997 & 0.000 & 0.022 & 0.000 & -0.003 & 0.000 \\
\hline 21 & 0.294 & 0.256 & -0.149 & -0.059 & -0.009 & 0.000 \\
\hline 22 & 0.479 & 0.574 & 0.086 & 0.000 & 0.031 & 0.000 \\
\hline 23 & 0.551 & 0.661 & -0.046 & 0.000 & -0.016 & 0.000 \\
\hline 24 & 0.430 & 0.367 & 0.042 & 0.000 & 0.021 & 0.000 \\
\hline 25 & 1.497 & 1.257 & 0.070 & 0.000 & 0.024 & 0.000 \\
\hline $26^{2}$ & -6.451 & -6.051 & 0.265 & 0.589 & 0.736 & 0.889 \\
\hline $\mathrm{E}\left(\mathrm{X}^{* \prime} \widetilde{R}\right)^{3}$ & 1.166 & 1.156 & 1.018 & 1.007 & 1.007 & 1.000 \\
\hline $\begin{array}{l}\text { Certainty } \\
\text { Equivalent }\end{array}$ & 1.088 & 1.077 & 1.009 & 0.999 & 1.001 & 0.993 \\
\hline
\end{tabular}

${ }^{I}$ Numbers are not comparable across levels of risk aversion, because for each level of risk aversion a different set of available assets was used.

${ }^{2}$ The $26^{\text {th }}$ asset is risk-free in nominal terms.

${ }^{3}$ Monthly gross expected returns on portfolios. 
number is just large enough to give an opportunity cost of no more than $1.0 \%$, the cut-off value after which the diversification benefits are assumed to be non-significant.

For Table 3 for risk aversion of 0.7 more than $100 \%$ of initial wealth, $w_{0}$, is held in the nominally risky assets (asset \#1 through asset \#25) as a group, and Treasury bills are held in negative quantities.

As risk aversion increases, as investors become more conservative and less risktolerant, the proportion of initial wealth held in Treasury bills becomes positive and increases, and correspondingly the proportion of initial wealth held in the group of nominally risky assets decreases.

The tables show that unconstrained and constrained optimal portfolio shares are not similar for different levels of risk aversion. As a matter of fact, optimal unconstrained and constrained portfolios for the low level of relative risk aversion of 0.7 have more extreme quantities (negative as well as positive) of assets than optimal unconstrained and constrained portfolios for the medium level of relative risk aversion of 11 and for the high level of relative risk aversion of 31 . Extremely negative quantities of assets for high risk-tolerance investors mean that the investors follow an aggressive short sale strategy.

Also Table 3 and Table 4 show monthly expected returns on unconstrained and constrained optimal portfolios, $E\left(X^{* \prime} \widetilde{R}\right)$, for the three levels of relative risk aversion. The expected returns for constrained and unconstrained optimal portfolios for risk aversion of 0.7 are very dramatic for the case with no short-selling constraint and somewhat large for the case with the short-selling constraint. Expected returns are of medium size for risk aversion of 11 and of small size for risk aversion of 31 for both cases. Such magnitudes of expected portfolio returns for high risk-tolerance investors 
Table 4

Illustrative optimal portfolio shares for unconstrained and optimally constrained to include $\boldsymbol{n}$ assets portfolio strategies for different values of relative risk aversion with the short-selling constraint ${ }^{1}$

\begin{tabular}{|c|c|c|c|c|c|c|}
\hline \multirow[t]{2}{*}{ Asset \# } & \multicolumn{2}{|c|}{$\begin{array}{l}\text { Relative Risk Aversion, }(1-\gamma) \text {, } \\
\text { equal to } 0.7\end{array}$} & \multicolumn{2}{|c|}{$\begin{array}{c}\text { Relative Risk Aversion, } \\
(1-\gamma) \text {, equal to } 11\end{array}$} & \multicolumn{2}{|c|}{$\begin{array}{c}\text { Relative Risk Aversion, } \\
(1-\gamma) \text {, equal to } 31\end{array}$} \\
\hline & Unconstrained & Constrained & Unconstrained & Constrained & Unconstrained & Constrained \\
\hline 1 & -0.010 & 0.000 & -0.020 & 0.000 & -0.020 & 0.000 \\
\hline 2 & -0.030 & -0.055 & -0.010 & 0.000 & 0.009 & 0.000 \\
\hline 3 & -0.020 & 0.000 & 0.176 & 0.000 & 0.065 & 0.000 \\
\hline 4 & -0.020 & 0.000 & -0.020 & 0.000 & -0.010 & 0.000 \\
\hline 5 & -0.010 & 0.000 & 0.009 & 0.000 & 0.026 & 0.000 \\
\hline 6 & -0.030 & 0.000 & 0.237 & 0.019 & 0.001 & 0.000 \\
\hline 7 & -0.020 & 0.000 & 0.019 & 0.000 & -0.009 & 0.000 \\
\hline 8 & -0.020 & 0.000 & -0.020 & 0.012 & -0.020 & 0.000 \\
\hline 9 & -0.050 & -0.055 & 0.015 & 0.000 & -0.020 & 0.000 \\
\hline 10 & 0.377 & 0.602 & 0.026 & 0.000 & 0.012 & 0.001 \\
\hline 11 & -0.010 & 0.000 & -0.020 & 0.000 & -0.006 & 0.000 \\
\hline 12 & -0.010 & 0.055 & -0.020 & 0.000 & -0.020 & 0.000 \\
\hline 13 & -0.010 & 0.000 & 0.061 & 0.000 & 0.139 & 0.003 \\
\hline 14 & -0.030 & 0.000 & 0.087 & 0.000 & -0.020 & 0.000 \\
\hline 15 & -0.010 & -0.055 & 0.024 & 0.000 & -0.001 & 0.000 \\
\hline 16 & 0.281 & 0.152 & 0.029 & 0.000 & 0.059 & 0.000 \\
\hline 17 & -0.030 & -0.055 & -0.020 & 0.000 & -0.020 & 0.000 \\
\hline 18 & -0.010 & 0.000 & -0.020 & 0.000 & 0.041 & 0.000 \\
\hline 19 & -0.040 & -0.055 & -0.020 & 0.000 & 0.012 & 0.000 \\
\hline 20 & -0.010 & 0.000 & -0.020 & 0.000 & -0.020 & 0.000 \\
\hline 21 & -0.010 & 0.000 & -0.020 & 0.000 & 0.008 & 0.000 \\
\hline 22 & -0.020 & 0.000 & -0.020 & 0.000 & 0.001 & 0.000 \\
\hline 23 & -0.020 & 0.000 & -0.019 & 0.000 & -0.005 & 0.000 \\
\hline 24 & -0.030 & -0.055 & 0.042 & 0.000 & -0.002 & 0.000 \\
\hline 25 & 0.802 & 0.686 & 0.181 & 0.000 & 0.018 & 0.000 \\
\hline $26^{2}$ & -0.060 & -0.115 & 0.331 & 0.969 & 0.769 & 0.996 \\
\hline $\mathrm{E}\left(\mathrm{X}^{* \prime} \widetilde{R}\right)^{3}$ & 1.056 & 1.042 & 1.017 & 1.006 & 1.006 & 1.000 \\
\hline $\begin{array}{c}\text { Certainty } \\
\text { Equivalent }\end{array}$ & 1.047 & 1.036 & 1.009 & 0.999 & 1.004 & 0.994 \\
\hline
\end{tabular}

${ }^{I}$ Numbers are not comparable across levels of risk aversion, because for each level of risk aversion a different set of available assets was used.

${ }^{2}$ The $13^{\text {th }}$ asset is risk-free in nominal terms.

${ }^{3}$ Monthly gross expected returns on portfolios. 
confirm the previously made conclusion about very aggressive short sale strategies. These magnitudes suggest very leveraged portfolios (unconstrained as well as constrained) for the case with no short-selling constraint. For investors with risk aversion of 11 and 31 there is, definitely, some short selling going on too for the case with no short-selling constraint and there is almost no short-selling going on for the case with the short-selling constraint. The less aggressive short selling for medium or high risk aversion leads to lower mean return portfolios.

There is a difference between the expected portfolio returns for the case with no short-selling constraint and for the case with the short-selling constraint. Expected portfolio returns are higher in the first case when there is no short-selling constraint involved. Table 4 shows that presence of the short-selling constraint has the result of investing in a smaller number of assets. But as the number of assets in investors' portfolios decreases with the constraint, the less opportunities investors have to seek higher mean returns while simultaneously decreasing diversification.

Table 3 and Table 4 also report the certainty equivalents calculated for the same three levels of relative risk aversion $(0.7,11$ and 31$)$. The certainty equivalent, $(C E)$, is defined by

$$
\frac{1}{\gamma} C E^{\gamma}=\frac{1}{\gamma} w_{0}^{\gamma} E\left(\widetilde{R}^{\gamma}\right)
$$

and so, with $w_{0}=1$,

$$
C E=\left(E\left[\widetilde{R}^{\gamma}\right]\right)^{\frac{1}{\gamma}} .
$$

The certainty equivalent represents the amount of certain wealth that would be viewed with indifference to the optimal portfolio. It is computed for investors of different 
levels of risk aversion: low (of 0.7), medium (of 11) and high (of 31). The two tables show that as risk aversion increases the value of the certainty equivalent decreases (for the unconstrained portfolio strategy as well as for the constrained) for both cases, with and without the short-selling constraint. This suggests that as investors become more afraid of risk they use less risky portfolio strategies and will be expecting lower returns from those portfolios and, so, the certain amount of wealth they will be willing to accept with indifference will decrease.

\subsubsection{Results Derived from Historical Returns Data with Extreme Returns Exaggerated}

In order to check the robustness of the estimates of the proportionate opportunity cost I include extremely high and extremely low simulated asset returns in each data set of available assets.

I construct the simulated extremely high and extremely low asset returns the following way. For the original data set for each historical time period I compute the average excess return across all assets in the data set. A historical time period with the highest average excess return across all assets defines the historical period with the highest returns. A historical time period with the lowest average excess return across all assets defines the historical period with the lowest returns. Then, for the extreme historical periods only I calculate the deviation of each asset's return from that asset's intertemporal mean return. The deviations are doubled and then added back to assets' intertemporal means. This way I create two fictional time periods with exaggerated high and exaggerated low returns. These fictional asset returns provide simulated extreme time 
periods to replace the time periods they were constructed from. The rest of the original data set remains unchanged.

Then I repeat the whole procedure of calculating the proportionate opportunity cost 1,000 times in each case simulating two fictional data periods. This gives me 1,000 new $\theta$ 's from the 1,000 data sets with extreme returns added. The results from this project are reported in Table 5.

For risk aversion of 0.7 the number of assets beyond which the opportunity cost is not higher than $1 \%$ of initial wealth is 25 . With 25 assets in the investor's portfolio it is no longer helpful to make more assets available for the portfolio.

The highest mean (over 1,000 replications) of the proportionate opportunity cost, $18.6 \%$, corresponds to the lowest level of risk aversion of 0.7 and will be incurred by an investor should he decide to invest in three assets rather than in 26 assets. The lowest mean (over 1,000 replications) of the proportionate opportunity cost, $0.0 \%$, corresponds to the highest level of risk aversion of 31 and will be incurred by an investor should he decide to invest in ten or more assets rather than in 26 .

The mean values (over 1,000 replications) of the proportionate opportunity cost of investing in four assets rather than 26 range from $16.8 \%$ for the level of risk aversion of 0.7 to $0.7 \%$ for the level of risk aversion of 31 . The mean values (over 1,000 replications) of the proportionate opportunity cost of investing in five assets rather than 26 range from $16.4 \%$ for the level of risk aversion of 0.7 to $0.6 \%$ for the level of risk aversion of 31 . The mean values (over 1,000 replications) of the proportionate opportunity cost of investing in ten assets rather than 26 range from $14.0 \%$ for the level of risk aversion of 
Table 5

The proportionate opportunity cost of restricting the number of assets in portfolios, $(\theta-1)$, for various values of relative risk aversion with extreme returns exaggerated in the asset returns distribution (with no short-selling constraint; mean values over 1,000 replications)

\begin{tabular}{|c|c|c|c|c|c|c|c|c|c|c|c|}
\hline $\begin{array}{c}\text { Number of } \\
\text { assets } \\
\text { in portfolios }\end{array}$ & 0.7 & 1 & 2 & 3 & 9 & 10 & 11 & 12 & 29 & 30 & 31 \\
\hline 3 & 0.186 & 0.165 & 0.142 & 0.123 & 0.064 & 0.056 & 0.043 & 0.031 & 0.016 & $\mathbf{0 . 0 1 0}$ & $\mathbf{0 . 0 0 9}$ \\
4 & 0.168 & 0.157 & 0.138 & 0.119 & 0.060 & 0.050 & 0.039 & 0.028 & $\mathbf{0 . 0 1 0}$ & 0.008 & 0.007 \\
5 & 0.164 & 0.149 & 0.130 & 0.111 & 0.057 & 0.045 & 0.030 & 0.021 & 0.009 & 0.007 & 0.006 \\
6 & 0.160 & 0.142 & 0.127 & 0.108 & 0.051 & 0.038 & 0.026 & 0.017 & 0.009 & 0.006 & 0.005 \\
7 & 0.155 & 0.136 & 0.123 & 0.103 & 0.048 & 0.029 & 0.018 & 0.012 & 0.008 & 0.005 & 0.003 \\
8 & 0.151 & 0.130 & 0.118 & 0.097 & 0.037 & 0.019 & 0.013 & $\mathbf{0 . 0 1 0}$ & 0.007 & 0.005 & 0.003 \\
9 & 0.143 & 0.128 & 0.115 & 0.090 & 0.026 & 0.014 & $\mathbf{0 . 0 1 0}$ & 0.007 & 0.005 & 0.003 & 0.001 \\
10 & 0.140 & 0.123 & 0.110 & 0.084 & 0.018 & $\mathbf{0 . 0 1 0}$ & 0.008 & 0.007 & 0.004 & 0.001 & 0.000 \\
11 & 0.133 & 0.117 & 0.107 & 0.081 & $\mathbf{0 . 0 1 0}$ & 0.008 & 0.008 & 0.006 & 0.003 & 0.000 & 0.000 \\
12 & 0.126 & 0.114 & 0.094 & 0.074 & 0.009 & 0.008 & 0.007 & 0.006 & 0.001 & 0.000 & 0.000 \\
13 & 0.118 & 0.108 & 0.090 & 0.070 & 0.008 & 0.008 & 0.006 & 0.004 & 0.000 & 0.000 & 0.000 \\
14 & 0.109 & 0.100 & 0.083 & 0.067 & 0.008 & 0.007 & 0.005 & 0.004 & 0.000 & 0.000 & 0.000 \\
15 & 0.101 & 0.089 & 0.078 & 0.058 & 0.006 & 0.006 & 0.005 & 0.003 & 0.000 & 0.000 & 0.000 \\
16 & 0.092 & 0.081 & 0.071 & 0.050 & 0.006 & 0.005 & 0.004 & 0.003 & 0.000 & 0.000 & 0.000 \\
17 & 0.084 & 0.074 & 0.065 & 0.043 & 0.005 & 0.005 & 0.003 & 0.001 & 0.000 & 0.000 & 0.000 \\
18 & 0.076 & 0.062 & 0.057 & 0.037 & 0.005 & 0.004 & 0.003 & 0.001 & 0.000 & 0.000 & 0.000 \\
19 & 0.065 & 0.050 & 0.046 & 0.025 & 0.004 & 0.003 & 0.001 & 0.000 & 0.000 & 0.000 & 0.000 \\
20 & 0.057 & 0.043 & 0.037 & 0.017 & 0.003 & 0.003 & 0.001 & 0.000 & 0.000 & 0.000 & 0.000 \\
21 & 0.043 & 0.034 & 0.023 & $\mathbf{0 . 0 1 0}$ & 0.003 & 0.001 & 0.000 & 0.000 & 0.000 & 0.000 & 0.000 \\
22 & 0.036 & 0.025 & 0.016 & 0.005 & 0.001 & 0.000 & 0.000 & 0.000 & 0.000 & 0.000 & 0.000 \\
23 & 0.024 & 0.017 & $\mathbf{0 . 0 1 0}$ & 0.003 & 0.000 & 0.000 & 0.000 & 0.000 & 0.000 & 0.000 & 0.000 \\
24 & 0.016 & $\mathbf{0 . 0 1 0}$ & 0.006 & 0.003 & 0.000 & 0.000 & 0.000 & 0.000 & 0.000 & 0.000 & 0.000 \\
25 & $\mathbf{0 . 0 1 0}$ & 0.006 & 0.003 & 0.001 & 0.000 & 0.000 & 0.000 & 0.000 & 0.000 & 0.000 & 0.000 \\
\hline
\end{tabular}

0.7 to $0.0 \%$ for the level of risk aversion of 31 . It is clear that the mean values of the proportionate opportunity cost of investing in $n$ assets rather than in 26 with extreme returns exaggerated in the returns distributions decrease as the number of assets available for investment increases, and as the level of relative risk aversion increases.

These results agree with the conclusion for the case with no short-selling constraint and with no exaggeration of extreme returns, and for the case with the short- 
selling constraint and with no exaggeration of extreme returns, that the mean values of the proportionate opportunity cost of investing in $n$ assets rather than in 26 decrease as the number of assets available for investment increases. As in the two previous cases the investor's marginal benefit from adding one more new asset to his portfolio decreases with every new asset. This marginal investor's benefit decreases more and more as more and more assets become available for investment.

The results from Table 5 also agree with another conclusion for the two previous cases that the mean values of the proportionate opportunity cost of investing in $n$ assets rather than in 26 decrease as the level of relative risk aversion increases. As in the two previous cases as the level of risk aversion increases, as investors become more and more afraid of risk, they will prefer to increase the fraction of their initial wealth placed in the nominally risk-free asset, Treasury bills, and whatever is left will be divided between nominally risky assets. So, even if they increase the number of nominally risky assets in their portfolios, the diversification benefit will not be as substantial as for investors with a lower level of risk aversion due to the smaller fraction of the initial wealth placed in the nominally risky assets. Therefore, the mean values of the proportionate opportunity cost of investing in $n$ assets rather than in 26 for investors with higher levels of risk aversion will be lower.

Even though, in general, results from Table 5 agree with results from Table 1 there is a subtle difference between these two tables. First of all, the well-diversified number of assets for every level of risk aversion is slightly smaller in Table 1 than in Table 5. Second, the magnitude of the proportionate opportunity cost of investing in $n$ assets rather than in 26 is slightly smaller in Table 1 than in Table 5. 
These differences can be explained by the following. Calculations of the proportionate opportunity cost and finding the well-diversified number of assets in Table 5 were based on the historical asset returns distribution with the extreme returns exaggerated. This exaggeration of historically occurred extreme returns converted the original asset returns distribution, derived as shown in (4)-(9), into distribution with fatter tails. This change in the distribution increases the probability for investors to end up with extremely low portfolio returns. Therefore, the countermeasure to fatter tails is to include more assets in the optimal unconstrained portfolios, to increase the diversification benefit. And that change in the optimal unconstrained portfolio strategy is reflected in Table 5 through a larger well-diversified number of assets in investors' portfolios for every level of risk aversion.

The magnitude of the proportionate opportunity cost is also affected by the change in the asset returns distribution. Now with fatter tails in the distribution it is even more costly to invest in a non-well-diversified number of assets than before, when investors were dealing with the original distribution, because a non-well-diversified number of assets dramatically increases the probability of ending up with extremely low portfolio returns.

\subsection{Results Derived for Opportunity Cost of Restricting the Number of Assets in Portfolios and of Using a Restricted VAR.}

This part of the section discusses the results derived from calculations of the proportionate opportunity costs that reflect not only the cost of restricting the number of assets in investors' portfolios but also the cost of using a restricted VAR. In the course of 
calculating these costs only a 26-asset VAR was used. Therefore, the expectations in (12) and (13) were taken over the distributions of asset returns implied by the same 26 -asset VAR for each set of available assets.

\subsubsection{Results Derived from Historical Returns Data with no Exaggeration of Extreme \\ Returns}

\subsubsection{Opportunity Costs with and without a Short-Selling Constraint}

Table 6 and Table 7 present the results from calculation of 1,000 values of the proportionate opportunity cost for each of 11 different values of relative risk aversion for portfolios with three to 25 assets with no short-selling constraint, and with three to 17 assets with the short-selling constraint, based on returns distributions derived from historically occurring asset returns over the 10-year period January 1992 through December 2001.

For relative risk aversion of 0.7 for the case with no short-selling constraint the number of assets beyond which the mean value (over 1,000 replications) of the opportunity cost is no higher than $1 \%$ of initial wealth is 25 . With 25 assets in the investor's portfolio for the risk aversion of 0.7 it is no longer helpful to make more assets available for the portfolio. As risk aversion increases, though, the well-diversified number of assets in investors' portfolios decreases. It reaches 24 assets for risk aversion of one; 23 assets for risk aversion of two; 21 assets for risk aversion of three; 11 assets for risk aversion of nine; four assets for investors with risk aversion of 29 and higher.

For the case with no short-selling constraint, as Table 6 shows, the highest mean (over 1,000 replications) of the proportionate opportunity cost, $17.6 \%(0.176)$, 
Table 6

The proportionate opportunity cost of restricting the number of assets in portfolios and of using a restricted VAR, $(\theta-1)$, for various values of relative risk aversion with no short-selling constraint (mean values over 1,000 replications)

\begin{tabular}{|c|c|c|c|c|c|c|c|c|c|c|c|}
\hline $\begin{array}{c}\text { Number of } \\
\text { assets } \\
\text { in portfolios }\end{array}$ & $\mathbf{0 . 7}$ & $\mathbf{1}$ & $\mathbf{2}$ & $\mathbf{3}$ & $\mathbf{9}$ & $\mathbf{1 0}$ & $\mathbf{1 1}$ & $\mathbf{1 2}$ & $\mathbf{2 9}$ & $\mathbf{3 0}$ & $\mathbf{3 1}$ \\
\hline $\mathbf{3}$ & 0.176 & 0.125 & 0.081 & 0.059 & 0.025 & 0.022 & 0.020 & 0.019 & 0.010 & 0.010 & 0.010 \\
$\mathbf{4}$ & 0.167 & 0.120 & 0.076 & 0.055 & 0.020 & 0.018 & 0.017 & 0.017 & $\mathbf{0 . 0 1 0}$ & $\mathbf{0 . 0 1 0}$ & $\mathbf{0 . 0 1 0}$ \\
$\mathbf{5}$ & 0.159 & 0.114 & 0.069 & 0.050 & 0.018 & 0.017 & 0.016 & 0.015 & 0.008 & 0.008 & 0.006 \\
$\mathbf{6}$ & 0.153 & 0.105 & 0.065 & 0.048 & 0.016 & 0.016 & 0.014 & 0.012 & 0.007 & 0.006 & 0.004 \\
$\mathbf{7}$ & 0.147 & 0.094 & 0.060 & 0.046 & 0.014 & 0.013 & 0.012 & 0.011 & 0.005 & 0.004 & 0.003 \\
$\mathbf{8}$ & 0.139 & 0.090 & 0.057 & 0.043 & 0.012 & 0.012 & 0.011 & 0.010 & 0.005 & 0.004 & 0.001 \\
$\mathbf{9}$ & 0.132 & 0.084 & 0.053 & 0.039 & 0.011 & 0.011 & 0.010 & $\mathbf{0 . 0 1 0}$ & 0.003 & 0.002 & 0.000 \\
$\mathbf{1 0}$ & 0.125 & 0.075 & 0.048 & 0.037 & 0.010 & 0.010 & $\mathbf{0 . 0 1 0}$ & 0.008 & 0.001 & 0.001 & 0.000 \\
$\mathbf{1 1}$ & 0.119 & 0.070 & 0.046 & 0.034 & $\mathbf{0 . 0 1 0}$ & $\mathbf{0 . 0 1 0}$ & 0.007 & 0.007 & 0.000 & 0.000 & 0.000 \\
$\mathbf{1 2}$ & 0.114 & 0.066 & 0.040 & 0.030 & 0.007 & 0.007 & 0.007 & 0.007 & 0.000 & 0.000 & 0.000 \\
$\mathbf{1 3}$ & 0.104 & 0.058 & 0.036 & 0.027 & 0.006 & 0.007 & 0.006 & 0.006 & 0.000 & 0.000 & 0.000 \\
$\mathbf{1 4}$ & 0.097 & 0.052 & 0.032 & 0.023 & 0.005 & 0.006 & 0.006 & 0.006 & 0.000 & 0.000 & 0.000 \\
$\mathbf{1 5}$ & 0.090 & 0.046 & 0.028 & 0.021 & 0.005 & 0.005 & 0.005 & 0.005 & 0.000 & 0.000 & 0.000 \\
$\mathbf{1 6}$ & 0.083 & 0.039 & 0.026 & 0.018 & 0.005 & 0.005 & 0.004 & 0.003 & 0.000 & 0.000 & 0.000 \\
$\mathbf{1 7}$ & 0.077 & 0.036 & 0.021 & 0.016 & 0.003 & 0.004 & 0.004 & 0.003 & 0.000 & 0.000 & 0.000 \\
$\mathbf{1 8}$ & 0.069 & 0.030 & 0.017 & 0.014 & 0.003 & 0.004 & 0.003 & 0.003 & 0.000 & 0.000 & 0.000 \\
$\mathbf{1 9}$ & 0.062 & 0.025 & 0.015 & 0.012 & 0.003 & 0.003 & 0.003 & 0.001 & 0.000 & 0.000 & 0.000 \\
$\mathbf{2 0}$ & 0.053 & 0.018 & 0.013 & 0.010 & 0.001 & 0.003 & 0.002 & 0.000 & 0.000 & 0.000 & 0.000 \\
$\mathbf{2 1}$ & 0.045 & 0.015 & 0.011 & $\mathbf{0 . 0 1 0}$ & 0.001 & 0.000 & 0.000 & 0.000 & 0.000 & 0.000 & 0.000 \\
$\mathbf{2 2}$ & 0.035 & 0.013 & 0.010 & 0.005 & 0.000 & 0.000 & 0.000 & 0.000 & 0.000 & 0.000 & 0.000 \\
$\mathbf{2 3}$ & 0.028 & 0.011 & $\mathbf{0 . 0 1 0}$ & 0.004 & 0.000 & 0.000 & 0.000 & 0.000 & 0.000 & 0.000 & 0.000 \\
$\mathbf{2 4}$ & 0.018 & $\mathbf{0 . 0 1 0}$ & 0.005 & 0.003 & 0.000 & 0.000 & 0.000 & 0.000 & 0.000 & 0.000 & 0.000 \\
$\mathbf{2 5}$ & $\mathbf{0 . 0 1 0}$ & 0.005 & 0.003 & 0.001 & 0.000 & 0.000 & 0.000 & 0.000 & 0.000 & 0.000 & 0.000 \\
\hline
\end{tabular}

corresponds to the lowest level of risk aversion of 0.7 and will be incurred by an investor should he decide to invest in three assets rather than in 26 assets. The lowest mean (over 1,000 replications) of the proportionate opportunity cost, $0.0 \%(0.000)$, corresponds to the highest level of risk aversion of 31 and will be incurred by an investor should he decide to invest in nine or more assets rather than in 26.

The mean values (over 1,000 replications) of the proportionate opportunity cost of investing in four assets rather than 26 range from $16.7 \%$ for the level of risk aversion 
Table 7

The proportionate opportunity cost of restricting the number of assets in portfolios and of using a restricted VAR, $(\theta-1)$, for various values of relative risk aversion with

the short-selling constraint (mean values over 1,000 replications)

\begin{tabular}{|c|c|c|c|c|c|c|c|c|c|c|c|}
\hline $\begin{array}{c}\text { Number of } \\
\text { assets } \\
\text { in portfolios }\end{array}$ & $\mathbf{0 . 7}$ & $\mathbf{1}$ & $\mathbf{2}$ & $\mathbf{3}$ & $\mathbf{9}$ & $\mathbf{1 0}$ & $\mathbf{1 1}$ & $\mathbf{1 2}$ & $\mathbf{2 9}$ & $\mathbf{3 0}$ & $\mathbf{3 1}$ \\
\hline $\mathbf{3}$ & 0.022 & 0.020 & 0.018 & 0.017 & 0.012 & 0.012 & 0.011 & 0.011 & $\mathbf{0 . 0 1 0}$ & $\mathbf{0 . 0 1 0}$ & $\mathbf{0 . 0 1 0}$ \\
$\mathbf{4}$ & 0.020 & 0.019 & 0.017 & 0.015 & 0.011 & 0.011 & $\mathbf{0 . 0 1 0}$ & $\mathbf{0 . 0 1 0}$ & 0.009 & 0.008 & 0.008 \\
$\mathbf{5}$ & 0.018 & 0.017 & 0.016 & 0.014 & $\mathbf{0 . 0 1 0}$ & $\mathbf{0 . 0 1 0}$ & 0.009 & 0.008 & 0.008 & 0.006 & 0.005 \\
$\mathbf{6}$ & 0.017 & 0.015 & 0.014 & 0.013 & 0.009 & 0.009 & 0.008 & 0.006 & 0.006 & 0.004 & 0.003 \\
$\mathbf{7}$ & 0.016 & 0.014 & 0.013 & 0.012 & 0.007 & 0.008 & 0.006 & 0.004 & 0.003 & 0.002 & 0.001 \\
$\mathbf{8}$ & 0.015 & 0.013 & 0.012 & 0.011 & 0.006 & 0.005 & 0.004 & 0.003 & 0.002 & 0.001 & 0.000 \\
$\mathbf{9}$ & 0.014 & 0.012 & 0.011 & $\mathbf{0 . 0 1 0}$ & 0.004 & 0.004 & 0.003 & 0.001 & 0.000 & 0.000 & 0.000 \\
$\mathbf{1 0}$ & 0.013 & 0.011 & $\mathbf{0 . 0 1 0}$ & 0.009 & 0.002 & 0.001 & 0.001 & 0.000 & 0.000 & 0.000 & 0.000 \\
$\mathbf{1 1}$ & 0.012 & 0.010 & 0.009 & 0.008 & 0.001 & 0.000 & 0.000 & 0.000 & 0.000 & 0.000 & 0.000 \\
$\mathbf{1 2}$ & 0.011 & $\mathbf{0 . 0 1 0}$ & 0.007 & 0.007 & 0.000 & 0.000 & 0.000 & 0.000 & 0.000 & 0.000 & 0.000 \\
$\mathbf{1 3}$ & $\mathbf{0 . 0 1 0}$ & 0.009 & 0.007 & 0.006 & 0.000 & 0.000 & 0.000 & 0.000 & 0.000 & 0.000 & 0.000 \\
$\mathbf{1 4}$ & 0.009 & 0.008 & 0.005 & 0.004 & 0.000 & 0.000 & 0.000 & 0.000 & 0.000 & 0.000 & 0.000 \\
$\mathbf{1 5}$ & 0.008 & 0.007 & 0.005 & 0.004 & 0.000 & 0.000 & 0.000 & 0.000 & 0.000 & 0.000 & 0.000 \\
$\mathbf{1 6}$ & 0.006 & 0.005 & 0.004 & 0.003 & 0.000 & 0.000 & 0.000 & 0.000 & 0.000 & 0.000 & 0.000 \\
$\mathbf{1 7}$ & 0.006 & 0.005 & 0.003 & 0.002 & 0.000 & 0.000 & 0.000 & 0.000 & 0.000 & 0.000 & 0.000 \\
$\mathbf{1 8}$ & 0.005 & 0.004 & 0.003 & 0.001 & 0.000 & 0.000 & 0.000 & 0.000 & 0.000 & 0.000 & 0.000 \\
$\mathbf{1 9}$ & 0.004 & 0.003 & 0.001 & 0.000 & 0.000 & 0.000 & 0.000 & 0.000 & 0.000 & 0.000 & 0.000 \\
$\mathbf{2 0}$ & 0.003 & 0.001 & 0.000 & 0.000 & 0.000 & 0.000 & 0.000 & 0.000 & 0.000 & 0.000 & 0.000 \\
$\mathbf{2 1}$ & 0.003 & 0.000 & 0.000 & 0.000 & 0.000 & 0.000 & 0.000 & 0.000 & 0.000 & 0.000 & 0.000 \\
$\mathbf{2 2}$ & 0.001 & 0.000 & 0.000 & 0.000 & 0.000 & 0.000 & 0.000 & 0.000 & 0.000 & 0.000 & 0.000 \\
$\mathbf{2 3}$ & 0.001 & 0.000 & 0.000 & 0.000 & 0.000 & 0.000 & 0.000 & 0.000 & 0.000 & 0.000 & 0.000 \\
$\mathbf{2 4}$ & 0.000 & 0.000 & 0.000 & 0.000 & 0.000 & 0.000 & 0.000 & 0.000 & 0.000 & 0.000 & 0.000 \\
$\mathbf{2 5}$ & 0.000 & 0.000 & 0.000 & 0.000 & 0.000 & 0.000 & 0.000 & 0.000 & 0.000 & 0.000 & 0.000 \\
\hline
\end{tabular}

of 0.7 to $1.0 \%$ for the level of risk aversion of 31 . The mean values (over 1,000 replications) of the proportionate opportunity cost of investing in five assets rather than 26 range from $15.9 \%$ for the level of risk aversion of 0.7 to $0.6 \%$ for the level of risk aversion of 31 . The mean values (over 1,000 replications) of the proportionate opportunity cost of investing in ten assets rather than 26 range from $12.5 \%$ for the level of risk aversion of 0.7 to $0.0 \%$ for the level of risk aversion of 31 . It is clear that the mean values of the proportionate opportunity cost of investing in $n$ assets rather than in 26 with 
no short-selling constraint decrease as the number of assets available for investment increases, and as the level of relative risk aversion increases.

With the short-selling constraint, for risk aversion of 0.7 the number of assets beyond which the mean value (over 1,000 replications) of the proportionate opportunity cost is no higher than $1 \%$ of initial wealth is 13 . It reaches 12 assets for risk aversion of one; ten assets for investors with risk aversion of two; nine assets for investors with risk aversion of three; five assets for investors with risk aversion of nine and ten; four assets for risk aversion of 11 and 12; and three or less assets for risk aversion of 29 and higher.

As Table 7 shows, for the case with the short-selling constraint, the highest mean (over 1,000 replications) of the proportionate opportunity cost, $2.2 \%(0,022)$, corresponds to the lowest level of risk aversion of 0.7 and will be incurred by an investor should he decide to invest in three assets rather than in 26 assets. The lowest mean (over 1,000 replications) of the proportionate opportunity cost, $0.0 \%(0.000)$, corresponds to the highest level of risk aversion of 31 and will be incurred by an investor should he decide to invest in eight or more assets rather than in 26.

The mean values (over 1,000 replications) of the proportionate opportunity cost of investing in four assets rather than 26 range from $2.0 \%$ for the level of risk aversion of 0.7 to $0.8 \%$ for the level of risk aversion of 31 . The mean values (over 1,000 replications) of the proportionate opportunity cost of investing in five assets rather than 26 range from $1.8 \%$ for the level of risk aversion of 0.7 to $0.5 \%$ for the level of risk aversion of 31 . The mean values (over 1,000 replications) of the proportionate opportunity cost of investing in ten assets rather than 26 range from $1.3 \%$ for the level of risk aversion of 0.7 to $0.0 \%$ for the level of risk aversion of 31. 
These results agree with the conclusion for the case with no short-selling constraint that the mean values of the proportionate opportunity cost of investing in $n$ assets rather than in 26 decrease as the number of assets available for investment increases. This conclusion also agrees with the one drawn for Tables 1-2. This makes sense. When one more asset becomes available for investment, it takes an investor's portfolio to a higher diversification level with lower unsystematic risk, and to a higher welfare level. But the investor's marginal benefit from adding one more new asset to his portfolio decreases with every new asset. This marginal investor's benefit decreases more and more as more and more assets become available for investment. That is why as the number of assets in investors' portfolios increases, the proportionate opportunity cost of not investing in 26 assets decreases.

The results from Table 7 also agree with another conclusion for the case with no short-selling constraint, that the mean value of the proportionate opportunity cost of investing in $n$ assets rather than in 26 decreases as the level of relative risk aversion increases. This conclusion also agrees with the one drawn for Tables 1-2. This also makes sense. As investors become more and more afraid of risk, they will prefer to increase the fraction of their initial wealth placed in the nominally risk-free asset, Treasury bills (see Table 8), and whatever is left will be divided between nominally risky assets. So, even if they increase the number of nominally risky assets in their portfolios, the diversification benefit will not be as substantial as for investors with a lower level of risk aversion due to the smaller fraction of the initial wealth placed in the nominally risky assets. Therefore, the mean values of the proportionate opportunity cost of investing in $n$ assets rather than in 26 for investors with higher levels of risk aversion will be lower. 
The only difference between Table 6 and Table 7 is that with the short-selling constraint the well-diversified number of assets in investors' portfolios is lower than that for portfolios with no short-selling constraint. And this is the result of placing the constraint on investors' behavior. This constraint will reduce any diversification benefits. The answer to the question of how much a short-selling constraint reduces investors' welfare due to the reduction of the diversification benefits will be answered in Chapter 4 of this dissertation.

Conclusions drawn for Tables 6-7 agree with those drawn for Tables 1-2. What differs though between these two pairs of tables is the magnitude of the mean values of the proportionate opportunity cost and the well-diversified number of assets. The mean values of the proportionate opportunity cost of investing in $n$ assets rather than in 26 are bigger for Tables 6-7 than those for Tables 1-2. This can be explained by the fact that the opportunity costs in Tables 6-7 reflect not only the cost of restricting the number of assets in investors' portfolios but also the cost of using a restricted VAR in derivations of the asset returns distribution functions. In calculations of mean values of the proportionate opportunity cost for Tables 1-2 two different VARs were used for deriving $n$-asset and 26-asset probability distribution functions for every set of available assets: a VAR for $n$ assets and a different VAR for 26 assets. In calculations of mean values of the proportionate opportunity cost for Tables 6-7 one VAR was used for deriving $n$-asset and 26-asset probability distribution functions for every set of available assets: a 26-asset VAR. Therefore, Tables 6-7 show not only the cost of sub-optimal diversification but also the cost of using the restricted VAR. 
As Tables 6-7 show, higher mean values (higher than those in Tables 1-2) of the proportionate opportunity cost of investing in $n$ assets rather than in 26 lead to a larger well-diversified number of assets in investors' portfolios. So, the result of using the unrestricted VAR is a higher diversification benefit due to a larger number of assets in an investor's portfolio.

\subsubsection{Optimal portfolio shares}

Table 8 and Table 9 present typical optimal portfolio shares for unconstrained (26-asset) and constrained portfolio strategies for three different levels of relative risk aversion: low (of 0.7), medium (of 11) and high (of 31). Table 8 presents optimal portfolio shares with no short-selling constraint; Table 9 presents optimal portfolio shares with a short-selling constraint. For all three levels of risk aversion there is a different well-diversified number of assets; therefore, constrained optimal portfolios for different levels of risk aversion have different numbers of assets in them. In each case illustrative optimal portfolio shares are calculated for a different set of available assets whose number is just large enough to give an opportunity cost of no more than $1.0 \%$, the cut-off value after which the diversification benefits are assumed to be non-significant.

For Table 8 for risk aversion of 0.7 more than $100 \%$ of initial wealth, $w_{0}$, is held in the nominally risky assets (asset \#1 through asset \#25) as a group, and Treasury bills are held in negative quantities.

As risk aversion increases, as investors become more conservative and less risktolerant, the proportion of initial wealth held in Treasury bills becomes positive and 
Table 8

Illustrative optimal portfolio shares for unconstrained and optimally constrained to include $\boldsymbol{n}$ assets and to use a restricted VAR portfolio strategies for different values of relative risk aversion ${ }^{1}$ (with no short-selling constraint)

\begin{tabular}{|c|c|c|c|c|c|c|}
\hline \multirow[t]{2}{*}{ Asset \# } & \multicolumn{2}{|c|}{$\begin{array}{l}\text { Relative Risk Aversion, }(1-\gamma) \text {, } \\
\text { equal to } 0.7\end{array}$} & \multicolumn{2}{|c|}{$\begin{array}{l}\text { Relative Risk Aversion, } \\
(1-\gamma) \text {, equal to } 11\end{array}$} & \multicolumn{2}{|c|}{$\begin{array}{l}\text { Relative Risk Aversion, } \\
(1-\gamma) \text {, equal to } 31\end{array}$} \\
\hline & Unconstrained & Constrained & Unconstrained & Constrained & Unconstrained & Constrained \\
\hline 1 & -1.308 & 0.000 & -0.136 & 0.000 & -0.002 & 0.000 \\
\hline 2 & 3.555 & 2.550 & -0.005 & 0.000 & 0.029 & 0.000 \\
\hline 3 & 1.127 & 0.764 & 0.109 & 0.024 & 0.223 & 0.054 \\
\hline 4 & 0.194 & 0.032 & -0.084 & -0.017 & -0.090 & -0.013 \\
\hline 5 & 0.584 & 0.402 & 0.218 & 0.000 & -0.013 & 0.000 \\
\hline 6 & 0.244 & 0.209 & 0.431 & 0.072 & -0.015 & 0.000 \\
\hline 7 & -0.377 & -0.328 & 0.023 & 0.000 & -0.019 & 0.000 \\
\hline 8 & 0.364 & 0.167 & -0.005 & 0.000 & -0.002 & 0.000 \\
\hline 9 & -1.794 & -1.486 & -0.041 & -0.021 & 0.016 & 0.000 \\
\hline 10 & 3.576 & 3.125 & 0.054 & 0.000 & 0.009 & 0.000 \\
\hline 11 & -1.105 & -1.369 & 0.130 & 0.000 & -0.003 & 0.000 \\
\hline 12 & -0.103 & 0.093 & 0.215 & 0.118 & 0.020 & 0.000 \\
\hline 13 & 0.147 & 0.389 & -0.085 & 0.000 & 0.029 & 0.000 \\
\hline 14 & 1.453 & 1.340 & 0.030 & 0.000 & 0.021 & 0.000 \\
\hline 15 & -1.974 & -1.298 & 0.128 & 0.022 & -0.049 & 0.000 \\
\hline 16 & -2.284 & -2.548 & 0.155 & 0.000 & 0.053 & 0.039 \\
\hline 17 & -0.879 & -1.019 & -0.007 & 0.000 & -0.004 & 0.000 \\
\hline 18 & 1.131 & 1.236 & 0.007 & 0.000 & 0.019 & 0.000 \\
\hline 19 & 3.750 & 3.290 & 0.071 & 0.000 & -0.013 & 0.000 \\
\hline 20 & 1.979 & 2.258 & -0.052 & -0.042 & 0.019 & 0.000 \\
\hline 21 & 2.026 & 1.897 & 0.121 & 0.078 & 0.007 & 0.000 \\
\hline 22 & 6.184 & 6.412 & 0.044 & 0.000 & 0.005 & 0.000 \\
\hline 23 & -3.326 & -1.295 & -0.058 & -0.031 & -0.034 & 0.000 \\
\hline 24 & -3.322 & -4.626 & 0.009 & 0.000 & 0.052 & 0.000 \\
\hline 25 & 1.526 & 1.369 & -0.022 & 0.000 & 0.081 & 0.000 \\
\hline $26^{2}$ & -10.369 & -10.566 & -0.250 & 0.797 & 0.663 & 0.919 \\
\hline $\mathrm{E}\left(\mathrm{X}^{* \prime} \widetilde{R}\right)^{3}$ & 1.341 & 1.324 & 1.026 & 1.011 & 1.010 & 1.001 \\
\hline $\begin{array}{c}\text { Certainty } \\
\text { Equivalent }\end{array}$ & 1.183 & 1.171 & 1.016 & 1.006 & 1.006 & 0.996 \\
\hline
\end{tabular}

${ }^{\prime}$ Numbers are not comparable across levels of risk aversion, because for each level of risk aversion a different set of available assets was used.

${ }^{2}$ The $26^{\text {th }}$ asset is risk-free in nominal terms.

${ }^{3}$ Monthly gross expected returns on portfolios. 
Table 9

Illustrative optimal portfolio shares for unconstrained and optimally constrained to include $\boldsymbol{n}$ assets and to use a restricted VAR portfolio strategies for different values of relative risk aversion with the short-selling constraint ${ }^{1}$

\begin{tabular}{|c|c|c|c|c|c|c|}
\hline \multirow[t]{2}{*}{ Asset \# } & \multicolumn{2}{|c|}{$\begin{array}{l}\text { Relative Risk Aversion, }(1-\gamma) \text {, } \\
\text { equal to } 0.7\end{array}$} & \multicolumn{2}{|c|}{$\begin{array}{l}\text { Relative Risk Aversion, } \\
(1-\gamma) \text {, equal to } 11\end{array}$} & \multicolumn{2}{|c|}{$\begin{array}{l}\text { Relative Risk Aversion, } \\
(1-\gamma) \text {, equal to } 31\end{array}$} \\
\hline & Unconstrained & Constrained & Unconstrained & Constrained & Unconstrained & Constrained \\
\hline 1 & -0.010 & 0.000 & 0.129 & 0.000 & \begin{tabular}{|l|}
-0.020 \\
\end{tabular} & 0.000 \\
\hline 2 & -0.020 & 0.000 & 0.027 & 0.000 & 0.007 & 0.000 \\
\hline 3 & -0.030 & -0.050 & 0.065 & 0.000 & -0.020 & 0.000 \\
\hline 4 & 0.392 & 0.443 & 0.023 & 0.000 & 0.028 & 0.118 \\
\hline 5 & 0.657 & 0.530 & 0.011 & 0.000 & 0.004 & 0.000 \\
\hline 6 & 0.172 & 0.392 & -0.020 & 0.000 & 0.073 & 0.000 \\
\hline 7 & -0.040 & -0.050 & -0.020 & 0.000 & -0.020 & -0.084 \\
\hline 8 & -0.010 & 0.000 & -0.020 & 0.000 & 0.042 & 0.000 \\
\hline 9 & -0.010 & 0.000 & 0.153 & 0.018 & 0.046 & 0.000 \\
\hline 10 & -0.020 & 0.000 & -0.020 & 0.000 & -0.020 & 0.000 \\
\hline 11 & -0.030 & -0.050 & -0.020 & 0.000 & -0.018 & 0.000 \\
\hline 12 & -0.010 & 0.000 & -0.020 & 0.000 & 0.029 & 0.000 \\
\hline 13 & -0.020 & 0.035 & 0.025 & 0.000 & 0.001 & 0.000 \\
\hline 14 & 0.160 & 0.000 & 0.136 & 0.000 & -0.004 & 0.000 \\
\hline 15 & -0.010 & 0.000 & -0.020 & 0.000 & 0.018 & 0.000 \\
\hline 16 & -0.010 & 0.000 & -0.020 & 0.000 & -0.009 & 0.000 \\
\hline 17 & -0.010 & -0.050 & 0.001 & 0.000 & 0.039 & 0.000 \\
\hline 18 & -0.050 & 0.000 & -0.020 & 0.000 & 0.025 & 0.000 \\
\hline 19 & -0.020 & 0.000 & -0.020 & 0.000 & -0.018 & 0.000 \\
\hline 20 & -0.020 & 0.000 & 0.119 & 0.000 & 0.016 & 0.000 \\
\hline 21 & -0.020 & 0.000 & 0.341 & 0.024 & 0.009 & 0.000 \\
\hline 22 & -0.010 & -0.050 & 0.149 & 0.000 & 0.018 & 0.000 \\
\hline 23 & -0.030 & -0.030 & -0.010 & -0.004 & 0.005 & 0.000 \\
\hline 24 & -0.020 & -0.010 & -0.020 & 0.000 & -0.020 & 0.000 \\
\hline 25 & 0.038 & 0.000 & -0.020 & 0.000 & 0.006 & 0.000 \\
\hline $26^{2}$ & -0.100 & -0.120 & 0.069 & 0.962 & 0.783 & 0.967 \\
\hline $\mathrm{E}\left(\mathrm{X}^{* \prime} \widetilde{R}\right)^{3}$ & 1.071 & 1.056 & 1.033 & 1.013 & 1.013 & 1.003 \\
\hline $\begin{array}{l}\text { Certainty } \\
\text { Equivalent }\end{array}$ & 1.049 & 1.039 & 1.020 & 1.010 & 1.008 & 0.998 \\
\hline
\end{tabular}

${ }^{\prime}$ Numbers are not comparable across levels of risk aversion, because for each level of risk aversion a different set of available assets was used.

${ }^{2}$ The $13^{\text {th }}$ asset is risk-free in nominal terms.

${ }^{3}$ Monthly gross expected returns on portfolios. 
increases, and correspondingly the proportion of initial wealth held in the group of nominally risky assets decreases.

The tables show that unconstrained and constrained optimal portfolio shares are not similar for different levels of risk aversion. As a matter of fact, optimal unconstrained and constrained portfolios for the low level of relative risk aversion of 0.7 have more extreme quantities (negative as well as positive) of assets than optimal unconstrained and constrained portfolios for the medium level of relative risk aversion of 11 and for the high level of relative risk aversion of 31. Extremely negative quantities of assets for high risk-tolerance investors mean that the investors follow an aggressive short sale strategy.

Also Table 8 and Table 9 show monthly expected returns on unconstrained and constrained optimal portfolios, $E\left(X^{* \prime} \widetilde{R}\right)$, for the three levels of relative risk aversion.

The expected returns for constrained and unconstrained optimal portfolios for risk aversion of 0.7 are very dramatic for the case with no short-selling constraint and somewhat large for the case with the short-selling constraint. Expected returns are of medium size for risk aversion of 11 and of small size for risk aversion of 31 for both cases. Such magnitudes of expected portfolio returns for high risk-tolerance investors confirm the previously made conclusion about very aggressive short sale strategies. These magnitudes suggest very leveraged portfolios (unconstrained as well as constrained) for the case with no short-selling constraint for investors with risk aversion of 0.7 . For investors with risk aversion of 11 and 31 there is, definitely, some short selling going on too for the case with no short-selling constraint and there is no shortselling going on at all for the short-sell constraint case. The less aggressive short selling for medium or high risk aversion leads to lower mean portfolio returns. 
There is a difference between the expected portfolio returns for the case with no short-selling constraint and for the case with the short-selling constraint. Expected portfolio returns are higher in the case with no short-selling constraint. Table 9 shows that presence of the short-selling constraint has the result of investing in a smaller number of assets. But as the number of assets in investors' portfolios decreases with the constraint, the less opportunities investors have to seek higher mean portfolio returns while simultaneously decreasing diversification.

Table 8 and Table 9 also report the certainty equivalents calculated for the same three levels of relative risk aversion $(0.7,11$ and 31$)$. The certainty equivalent represents the amount of certain wealth that would be viewed with indifference to the optimal portfolio. It is computed for investors of different levels of risk aversion: low (of 0.7), medium (of 11) and high (of 31). The two tables show that as risk aversion increases the value of the certainty equivalent decreases (for the unconstrained portfolio strategy as well as for the constrained) for both cases, with and without the short-selling constraint. This suggests that as investors become more afraid of risk they use less risky portfolio strategies and will be expecting lower portfolio returns and, so, the certain amount of wealth they will be willing to accept with indifference will decrease.

Conclusions drawn for Tables 8-9 agree with those drawn for Tables 3-4. What differs between these two pairs of tables are the magnitude of the expected portfolio returns and the certainty equivalent values, unconstrained as well as constrained. The expected portfolio returns and the certainty equivalent values are substantially bigger in Tables 8-9 for investors with risk aversion of 0.7 , somewhat bigger for investors with risk aversion of 11, and almost the same for investors with risk aversion of 31 than those in 
Tables 3-4. This can be explained by the fact that the well-diversified number of assets is larger now, Tables 6-7, for investors of almost all levels of risk aversion than it was before, Tables 1-2. A larger well-diversified number of assets means a higher level of diversification that offers a greater opportunity for an investor to seek higher portfolio returns. Higher expected portfolio returns will lead to higher certainty equivalents for investors.

\subsubsection{Results Derived from Historical Returns Data with Extreme Returns Exaggerated}

In order to check the robustness of the estimates of the proportionate opportunity cost I include extremely high and extremely low simulated asset returns in each data set of available assets. The same procedure as described earlier in this essay is used for calculating extremely low and extremely high simulated asset returns. The results from this project are reported in Table 10.

For risk aversion of 0.7 the number of assets beyond which the opportunity cost is not higher than $1 \%$ of initial wealth is 25 . With 25 assets in the investor's portfolio it is no longer helpful to make more assets available for the portfolio.

The highest mean (over 1,000 replications) of the proportionate opportunity cost, $18.6 \%(0.186)$, corresponds to the lowest level of risk aversion of 0.7 and will be incurred by an investor should he decide to invest in three assets rather than in 26 assets. The lowest mean (over 1,000 replications) of the proportionate opportunity cost, $0.0 \%$ (0.000), corresponds to the highest level of risk aversion of 31 and will be incurred by an investor should he decide to invest in 11 or more assets rather than in 26. 
Table 10

The proportionate opportunity cost of restricting the number of assets in portfolios an of using a restricted VAR, $(\theta-1)$, for various values of relative risk aversion with extreme returns exaggerated in the asset returns distribution (with no short-selling constraint; mean values over 1,000 replications)

\begin{tabular}{|c|c|c|c|c|c|c|c|c|c|c|c|}
\hline $\begin{array}{c}\text { Number of } \\
\text { assets } \\
\text { in portfolios }\end{array}$ & $\mathbf{0 . 7}$ & $\mathbf{1}$ & $\mathbf{2}$ & $\mathbf{3}$ & $\mathbf{9}$ & $\mathbf{1 0}$ & $\mathbf{1 1}$ & $\mathbf{1 2}$ & $\mathbf{2 9}$ & $\mathbf{3 0}$ & $\mathbf{3 1}$ \\
\hline $\mathbf{3}$ & 0.186 & 0.173 & 0.153 & 0.137 & 0.074 & 0.060 & 0.050 & 0.035 & 0.018 & 0.011 & 0.011 \\
$\mathbf{4}$ & 0.171 & 0.166 & 0.144 & 0.130 & 0.060 & 0.050 & 0.037 & 0.027 & $\mathbf{0 . 0 1 0}$ & $\mathbf{0 . 0 1 0}$ & $\mathbf{0 . 0 1 0}$ \\
$\mathbf{5}$ & 0.166 & 0.150 & 0.132 & 0.121 & 0.056 & 0.043 & 0.028 & 0.020 & 0.007 & 0.007 & 0.006 \\
$\mathbf{6}$ & 0.159 & 0.143 & 0.121 & 0.110 & 0.044 & 0.032 & 0.020 & 0.016 & 0.006 & 0.006 & 0.005 \\
$\mathbf{7}$ & 0.150 & 0.137 & 0.110 & 0.103 & 0.037 & 0.022 & 0.017 & 0.014 & 0.005 & 0.005 & 0.005 \\
$\mathbf{8}$ & 0.144 & 0.124 & 0.105 & 0.096 & 0.028 & 0.018 & 0.013 & 0.011 & 0.005 & 0.005 & 0.004 \\
$\mathbf{9}$ & 0.139 & 0.113 & 0.092 & 0.090 & 0.020 & 0.015 & 0.011 & $\mathbf{0 . 0 1 0}$ & 0.004 & 0.005 & 0.003 \\
$\mathbf{1 0}$ & 0.131 & 0.106 & 0.087 & 0.082 & 0.016 & 0.012 & $\mathbf{0 . 0 1 0}$ & 0.007 & 0.003 & 0.003 & 0.001 \\
$\mathbf{1 1}$ & 0.122 & 0.095 & 0.082 & 0.075 & 0.011 & $\mathbf{0 . 0 1 0}$ & 0.007 & 0.006 & 0.003 & 0.003 & 0.000 \\
$\mathbf{1 2}$ & 0.117 & 0.089 & 0.077 & 0.070 & $\mathbf{0 . 0 1 0}$ & 0.007 & 0.006 & 0.006 & 0.001 & 0.001 & 0.000 \\
$\mathbf{1 3}$ & 0.107 & 0.087 & 0.070 & 0.062 & 0.007 & 0.005 & 0.005 & 0.005 & 0.001 & 0.000 & 0.000 \\
$\mathbf{1 4}$ & 0.102 & 0.081 & 0.064 & 0.056 & 0.005 & 0.005 & 0.005 & 0.004 & 0.000 & 0.000 & 0.000 \\
$\mathbf{1 5}$ & 0.095 & 0.072 & 0.057 & 0.050 & 0.005 & 0.004 & 0.004 & 0.003 & 0.000 & 0.000 & 0.000 \\
$\mathbf{1 6}$ & 0.085 & 0.065 & 0.050 & 0.042 & 0.004 & 0.004 & 0.003 & 0.001 & 0.000 & 0.000 & 0.000 \\
$\mathbf{1 7}$ & 0.077 & 0.058 & 0.044 & 0.037 & 0.004 & 0.003 & 0.002 & 0.001 & 0.000 & 0.000 & 0.000 \\
$\mathbf{1 8}$ & 0.071 & 0.050 & 0.037 & 0.032 & 0.003 & 0.001 & 0.001 & 0.000 & 0.000 & 0.000 & 0.000 \\
$\mathbf{1 9}$ & 0.063 & 0.044 & 0.033 & 0.027 & 0.003 & 0.001 & 0.001 & 0.000 & 0.000 & 0.000 & 0.000 \\
$\mathbf{2 0}$ & 0.055 & 0.036 & 0.027 & 0.022 & 0.001 & 0.000 & 0.000 & 0.000 & 0.000 & 0.000 & 0.000 \\
$\mathbf{2 1}$ & 0.046 & 0.030 & 0.020 & 0.015 & 0.001 & 0.000 & 0.000 & 0.000 & 0.000 & 0.000 & 0.000 \\
$\mathbf{2 2}$ & 0.038 & 0.021 & 0.016 & $\mathbf{0 . 0 1 0}$ & 0.000 & 0.000 & 0.000 & 0.000 & 0.000 & 0.000 & 0.000 \\
$\mathbf{2 3}$ & 0.029 & 0.015 & $\mathbf{0 . 0 1 0}$ & 0.004 & 0.000 & 0.000 & 0.000 & 0.000 & 0.000 & 0.000 & 0.000 \\
$\mathbf{2 4}$ & 0.015 & $\mathbf{0 . 0 1 0}$ & 0.005 & 0.002 & 0.000 & 0.000 & 0.000 & 0.000 & 0.000 & 0.000 & 0.000 \\
$\mathbf{2 5}$ & $\mathbf{0 . 0 1 0}$ & 0.004 & 0.003 & 0.001 & 0.000 & 0.000 & 0.000 & 0.000 & 0.000 & 0.000 & 0.000 \\
\hline
\end{tabular}

The mean values (over 1,000 replications) of the proportionate opportunity cost of investing in four assets rather than 26 range from $17.1 \%$ for the level of risk aversion of 0.7 to $1.0 \%$ for the level of risk aversion of 31 . The mean values (over 1,000 replications) of the proportionate opportunity cost of investing in five assets rather than 26 range from $16.6 \%$ for the level of risk aversion of 0.7 to $0.6 \%$ for the level of risk aversion of 31 . The mean values (over 1,000 replications) of the proportionate opportunity cost of 
investing in ten assets rather than 26 range from $13.1 \%$ for the level of risk aversion of 0.7 to $0.1 \%$ for the level of risk aversion of 31 . It is clear that the mean values of the proportionate opportunity cost of investing in $n$ assets rather than in 26 with extreme returns exaggerated in the returns distributions decrease as the number of assets available for investment increases, and as the level of relative risk aversion increases.

These results agree with the conclusion for the case with no short-selling constraint and with no exaggeration of extreme returns (Tables 6), and for the case with the short-selling constraint and with no exaggeration of extreme returns (Table 7), that the mean values of the proportionate opportunity cost of investing in $n$ assets rather than in 26 decrease as the number of assets available for investment increases. As in the two previous cases the investor's marginal benefit from adding one more new asset to his portfolio decreases with every new asset. This marginal investor's benefit decreases more and more as more and more assets become available for investment.

The results from Table 10 also agree with another conclusion for the two previous cases that the mean values of the proportionate opportunity cost of investing in $n$ assets rather than in 26 decrease as the level of relative risk aversion increases. As in the two previous cases as the level of risk aversion increases, as investors become more and more afraid of risk, they will prefer to increase the fraction of their initial wealth placed in the nominally risk-free asset, Treasury bills, and whatever is left will be divided between nominally risky assets. So, even if they increase the number of nominally risky assets in their portfolios, the diversification benefit will not be as substantial as for investors with a lower level of risk aversion due to the smaller fraction of the initial wealth placed in the nominally risky assets. Therefore, the mean values of the proportionate opportunity cost 
of investing in $n$ assets rather than in 26 for investors with higher levels of risk aversion will be lower.

Even though, in general, results from Table 10 agree with results from Table 6 there is a subtle difference between these two tables. First of all, the optimal number of assets for several levels of risk aversion is slightly smaller in Table 6 than in Table 10 . Second, the magnitude of the proportionate opportunity cost of investing in $n$ assets rather than in 26 is slightly smaller in Table 6 than in Table 10.

These differences can be explained by the following. Calculation of the proportionate opportunity cost and finding the well-diversified number of assets in Table 10 were based on the historical asset returns distribution with the extreme returns exaggerated. This exaggeration of historically occurred extreme returns converted the original asset returns distribution, derived as shown in (4)-(9), into distribution with fatter tails. This change in the distribution increases the probability for investors to end up with extremely low portfolio returns. Therefore, the countermeasure to fatter tails is to include more assets in the optimal unconstrained portfolios, to increase the diversification benefit. And that change in the optimal unconstrained portfolio strategy is reflected in Table 10 through a larger well-diversified number of assets in investors' portfolios for every level of risk aversion.

The magnitude of the proportionate opportunity cost is also affected by the change in the asset returns distribution. Now with fatter tails in the distribution it is even more costly to invest in a non-well-diversified number of assets than before, when investors were dealing with the original distribution, because a non-well-diversified 
number of assets dramatically increases the probability of ending up with extremely low portfolio returns.

Conclusions drawn for Table 10 agree with those drawn for Table 5. What differs though between these two tables are the magnitudes of the mean values of the proportionate opportunity cost and the well-diversified number of assets for some levels of risk aversion. With extreme returns exaggerated in the asset returns distributions the mean values of the proportionate opportunity cost of investing in $n$ assets rather than in 26 are bigger for Table 10 than those for Table 5. This can be explained by the fact, again, that the opportunity costs in Table 10 reflect not only the cost of restricting the number of assets in investors' portfolios but also the cost of using a restricted VAR in derivations of the asset returns distribution functions. In the case of using the unrestricted VAR exaggeration of extreme returns in the asset returns distribution function will make those distribution tails even fatter than they were in the case of using the restricted VAR. Therefore, the cost of diversifying sub-optimally now is even higher. And the welldiversified number of assets is also larger now for investors with level of risk aversion of three, nine through 12,30 and 31.

\subsection{Summary of Results on Costs of Restricting the Number of Assets}

In this section of this essay I have investigated the opportunity cost incurred by investors when they invest in a non-well-diversified number of assets. CRRA utility functions and the proportionate opportunity cost have been used. The opportunity cost has been calculated for different values of relative risk aversion (including extreme levels of relative risk aversion) with and without a short-selling constraint. In addition, in the 
case with no short-selling constraint the analysis has been redone to find the effect of exaggerating extreme returns in the historical data.

The analysis has been done to find (a) the effect of restricting the number of assets in investors' portfolios, and (b) the effect of restricting the number of assets and of using a restricted VAR.

I found that the well-diversified number of assets depends on degree of risk aversion, presence or absence of the short-selling constraint, and the way the VAR process was used in deriving the asset returns distribution functions for the purpose of evaluating the opportunity cost.

The largest well-diversified number of assets found is 25 and it is for investors with risk aversion of 0.7 for the historical asset returns with extreme returns exaggerated in the asset returns distributions for the case with the restricted VAR. The same welldiversified number of assets was found to be the largest for investors with the same level of risk aversion for the case with no short-selling constraint, with no exaggeration of extreme returns in the asset returns distribution, and with unrestricted VAR. The lowest well-diversified number of assets found is three or less assets for investors with risk aversion 29 and higher for the historical asset returns data set with no exaggeration of extreme returns but with the short-selling constraint for the restricted VAR case. The same lowest well-diversified number of assets was found for unrestricted VAR case.

For all three cases with restricted VAR (original historical asset returns with no exaggeration of extreme returns and with no short-selling constraint, original historical asset returns with no exaggeration of extreme returns and with the short-selling constraint, and for the data set with extreme returns exaggerated and no short-selling 
constraint) as the level of relative risk aversion increases the proportionate opportunity cost of investing in $n$ assets rather than in 26 decreases, as well as the well-diversified number of assets. The same conclusions were drawn for those three cases but with unrestricted VAR.

There is a difference in magnitude of the proportionate opportunity cost and in the well-diversified number of assets for the data set with no exaggeration of extreme returns and that with extreme returns exaggerated for the restricted VAR case. The welldiversified number of assets and the proportionate opportunity cost of investing in $n$ assets rather than in 26 are bigger for the data set with extreme returns exaggerated. This can be explained by the fact that the presence of exaggerated extreme returns in the probability distribution produces fatter tails. These fatter tails in the probability distribution increase the chances of investors ending up with extremely low portfolio returns. That disturbs investors more than increased chances of ending up with extremely high portfolio returns pleases them. Therefore, the countermeasure to fatter tails is to include more assets in the optimal unconstrained portfolios, to increase the diversification benefit. Those fatter tails in the distribution also will increase the cost of diversifying sub-optimally. This is reflected in higher proportionate opportunity cost of investing in $n$ assets rather than in 26 .

My calculations showed that these conclusions and explanations also hold for data sets with and without exaggeration of extreme returns in the asset returns distributions with unrestricted VAR.

There is a subtle difference though in the magnitude of the proportionate opportunity costs and in the well-diversified number of assets for the restricted and 
unrestricted VARs cases. For the unrestricted VAR case, as my calculations showed, the opportunity costs are higher than those for the restricted VAR case. This can be explained by the fact that the opportunity costs for the unrestricted VAR case reflect not only the cost of restricting the number of assets in investors' portfolios but also the cost of using a restricted VAR in derivations of the asset returns distribution functions. In calculations of the proportionate opportunity costs for the restricted VAR case two different VARs were used for deriving $n$-asset and 26-asset probability distribution functions for each set of available assets: a VAR for $n$ assets and a different VAR for 26 assets. In calculations of the proportionate opportunity costs for the unrestricted VAR case only one VAR was used for deriving both $n$-asset and 26-asset probability distribution functions for each set of available assets: a 26-asset VAR. Therefore, the proportionate opportunity cost in the unrestricted VAR case shows not only the cost of sub-optimal diversification but also the cost of using the restricted VAR.

Also, higher proportionate opportunity costs of investing in $n$ assets rather than in 26 in the unrestricted VAR case lead to a larger well-diversified number of assets in investors' portfolios.

Therefore, based on my calculations, I may conclude that there is definitely a cost for investors to incur should they decide to invest in a non-well-diversified number of assets. This cost decreases as the non-well-diversified number of assets gets closer and closer to the well-diversified number of assets. And as far as my calculations show, only investors with very high levels of relative risk aversion (29 and above) will incur very small costs. Those investors will place such a big proportion of their initial wealth into 
nominally risk-free assets that it will not matter much how many nominally risky assets they have gotten in their portfolios.

\section{Opportunity Cost of Investing in Stocks only or Bonds only Versus Investing in both}

\section{Stocks and Bonds.}

There is a particular interesting case of being constrained by the number of assets in one's portfolio that I work with in this section of the essay. It is the case when a person invests in a portfolio that consists of stocks only or bonds only as opposed to a portfolio that consists of both stocks and bonds.

One motivation for doing this special case comes from Haliassos and Bertaut (1995). The authors investigated why many people do not hold stocks in their portfolios. In the article they pointed out that: “...[b]etween $75 \%$ and $80 \%$ of United States households ... do not hold stocks directly. This proportion is remarkably stable through time and across data bases". They suggested several reasons to explain the puzzle (e.g. costly information concerning the process of investing in stocks, education, cultural factors) and found support for their ideas through empirical studies.

But the question that remains unanswered in the article and the question I am interested is: how big is one's welfare loss if one restricts oneself to only one type of assets, i.e. bonds only or stocks only, rather than to have a portfolio of bonds and stocks together.

The same procedures as described earlier in this essay are used for calculating asset returns distributions and the proportionate opportunity costs, but now assets are stock and bond indices rather then individual stocks; and the numbers of assets combined 
are three (two stock indices and Treasury bills or two bond indices and Treasury bills) and five (two stock indices, two bond indices, and Treasury bills).

To form the "only stocks" constrained portfolio I use two composite stock indices: S\&P 500 and NASDAQ, with Treasury bills as the nominally risk-free asset. To form the "only bonds" constrained portfolio I use two composite bond indices: Salomon Brothers' Long-Term High-Grade Corporate Bonds Index and Long-Term Government Bonds Total Return index, with Treasury bills as the nominally risk-free asset. The unconstrained portfolio includes both types of assets: stocks and bonds. To form the unconstrained portfolio I use the same two composite stock indices and the same two composite bond indices, and Treasury bills.

\subsection{Vector Autoregressions of Returns}

To get expected values and probability distributions of real returns for the four indices and Treasury bills at time $T+1$, the portfolio formation period, I estimate a vector autoregressive process (VAR) exactly as previously described in this essay. As before the vector time series of residuals serves as the set of possible shocks to the projected mean vector of returns.

\subsection{Constrained Portfolios}

Using the information about the four indices and Treasury bills' derived probability distribution of real returns, I compute constrained optimal portfolios with (a) the two stock indices and Treasury bills, and (b) with the two bond indices and Treasury bills: the solutions of 


$$
\underset{\left\{\alpha_{1}, \alpha_{2}\right\}}{\operatorname{Max}} E U(\widetilde{w})=\operatorname{Max} E\left\{\frac{1}{\gamma}\left[w_{0}\left(\alpha_{1} \widetilde{r}_{S \& P 500}+\alpha_{2} \widetilde{r}_{N A S D A Q}+\left(1-\alpha_{1}-\alpha_{2}\right) \widetilde{r}_{T B}\right)\right]^{\gamma}\right\}
$$

(18) $\underset{\left\{\beta_{1}, \beta_{2}\right\}}{\operatorname{Max}} E U(\widetilde{w})=\operatorname{Max} E\left\{\frac{1}{\gamma}\left[w_{0}\left(\beta_{1} \widetilde{r}_{\text {Corporate Bonds }}+\beta_{2} \widetilde{r}_{\text {Government Bonds }}+\left(1-\beta_{1}-\beta_{2}\right) \widetilde{r}_{T B}\right)\right]^{\gamma}\right\}$

where $\alpha_{1}, \alpha_{2}, 1-\alpha_{1}-\alpha_{2}$ and $\beta_{1}, \beta_{2}, 1-\beta_{1}-\beta_{2}$ are the individual portfolio shares in the constrained optimal portfolios with stocks only and bonds only. To get these portfolios I search over $\alpha_{1}, \alpha_{2}$ and $\beta_{1}, \beta_{2}$ spaces to optimize expected utility, using nonlinear optimization by a quasi-Newton method based on convergence to first-order conditions of problem (17) and (18). The expectations are taken over the joint probability distributions derived from the 3-asset VAR.

\subsection{Unconstrained Portfolios}

The next step, then, is to get the unconstrained optimal portfolio with four indices and Treasury bills: the solution of

(19) $\underset{\left\{\alpha_{1}, \ldots, \alpha_{4}\right\}}{\operatorname{Max}} \operatorname{EU}(\widetilde{w})=$

$=\operatorname{Max} E\left\{\frac{1}{\gamma}\left[w_{0}\left(\kappa_{1} \widetilde{r}_{\text {S\&P500 }}+\kappa_{2} \widetilde{r}_{\text {NASDAQ }}+\kappa_{3} \widetilde{r}_{\text {Corporate Bonds }}+\kappa_{4} \widetilde{r}_{\text {Government Bonds }}+\left(1-\kappa_{1}-\ldots-\kappa_{4}\right) \widetilde{r}_{T B}\right)\right]^{\gamma}\right\}$

where $\kappa_{1}, \ldots, \kappa_{4}$ are the four individual indices' portfolio shares in the unconstrained optimal portfolio. To get the portfolio I search over $\kappa_{1}, \ldots, \kappa_{4}$ space to optimize expected utility, again using nonlinear optimization by a quasi-Newton method based on convergence to first-order conditions of problem (19). The expectation is taken over the joint probability distribution derived from the 5 -asset VAR. 


\subsection{Calculating Opportunity Cost}

Now, when I have the constrained and unconstrained optimal portfolios I calculate the proportionate opportunity cost, $\theta-1.0$. For the formulas for $\theta$ I need to find $\mathrm{E}\left(\widetilde{R}^{\gamma}\right)^{\text {unconstrained }}$ (where $\widetilde{R}$ is the gross return for the optimal unconstrained portfolio with four indices and Treasury bills), and $\mathrm{E}\left(\widetilde{R}_{\text {Bonds }}^{\gamma}\right)^{\text {constrained }}$ and $\mathrm{E}\left(\widetilde{R}_{\text {Stocks }}^{\gamma}\right)^{\text {constrained }}$ (where $\widetilde{R}_{\text {Bonds }}$ and $\widetilde{R}_{\text {Stocks }}$ are the gross returns for the optimal constrained portfolios with either two bonds indices and Treasury bills or with two stock indices and Treasury bills). $\mathrm{E}\left(\widetilde{R}^{\gamma}\right)^{\text {unconstrained }}$ will be equal to:

(20) $\mathrm{E}\left(\widetilde{R}^{\gamma}\right)^{\mathrm{uncd}}=\frac{1}{T} \sum_{t=1}^{T}\left\{\left[\begin{array}{lllll}\kappa_{1}^{*} & \kappa_{2}^{*} & \kappa_{3}^{*} & \kappa_{4}^{*} & \kappa_{5}^{*}\end{array}\right]\left[\begin{array}{c}E_{T} r_{S \& P 500, T+1}+\varepsilon_{S \& P 500, t}-\varepsilon_{\pi, t} \\ E_{T} r_{N A S D A Q, T+1}+\varepsilon_{N A S D A Q, t}-\varepsilon_{\pi, t} \\ E_{T} r_{\text {CorporateBonds }, T+1}+\varepsilon_{\text {CorporateBonds }, t}-\varepsilon_{\pi, t} \\ E_{T} r_{\text {Government Bonds }, T+1}+\varepsilon_{\text {Government Bonds }, t}-\varepsilon_{\pi, t} \\ E_{T} r_{T B, T+1}+0-\varepsilon_{\pi, t}\end{array}\right]\right\}$

where vector of $\kappa_{i}^{*}$ is the vector of optimal shares for unconstrained portfolio (with $\left.\kappa_{5}=1-\kappa_{1}-\ldots-\kappa_{4}\right)$; the vectors of $E_{T} r_{i, T+1}+\varepsilon_{i, t}-\varepsilon_{\pi, t}$ and $E_{T} r_{T B, T+1}+0-\varepsilon_{\pi, t}$ for $t=1, \ldots, T$ are the vectors of particular possible values of real returns (conditional on the data set for times $t=1$ through $T$ ) at time $T+1$.

And $E\left(\widetilde{R}^{\gamma}\right)^{\text {constrained }}$ will be equal to alternatively:

$$
E\left(\widetilde{R}_{\text {Stocks }}^{\gamma}\right)^{\text {constrained }}=\frac{1}{T} \sum_{t=1}^{T}\left\{\left[\begin{array}{lll}
\alpha_{1}^{*} & \alpha_{2}^{*} & \alpha_{3}^{*}
\end{array}\right]\left[\begin{array}{c}
E_{T} r_{S \& P 500, T+1}+\varepsilon_{S \& P 500, t}-\varepsilon_{\pi, t} \\
E_{T} r_{N A S D A Q, T+1}+\varepsilon_{N A S D A Q, t}-\varepsilon_{\pi, t} \\
E_{T} r_{T B, T+1}+0-\varepsilon_{\pi, t}
\end{array}\right]\right\}^{\gamma}
$$

and 


$$
E\left(\widetilde{R}_{\text {Bonds }}^{\gamma}\right)^{\text {consd }}=\frac{1}{T} \sum_{t=1}^{T}\left\{\left[\begin{array}{lll}
\beta_{1}^{*} & \beta_{2}^{*} & \beta_{3}^{*}
\end{array}\right]\left[\begin{array}{c}
E_{T} r_{\text {CorporateBonds }, T+1}+\varepsilon_{\text {CorporateBonds }, t}-\varepsilon_{\pi, t} \\
E_{T} r_{\text {Government Bonds }, T+1}+\varepsilon_{\text {Government Bonds }, t}-\varepsilon_{\pi, t} \\
E_{T} r_{T B, T+1}+0-\varepsilon_{\pi, t}
\end{array}\right]\right\}^{\gamma}
$$

where the vectors of $\alpha_{i}^{*}$ and $\beta_{i}^{*}$ are the unit-sum vectors of constrained portfolio shares for the "only stocks" portfolio and for the "only bonds" portfolio.

Finally, having calculated (21)-(22), I use (23) and (24) to get a numerical value for $\theta$ :

$$
\begin{aligned}
& \theta=\left[\frac{E\left(\widetilde{R}_{\text {Bonds and Stocks }}^{\gamma}\right)^{\text {optimal }}}{E\left(\widetilde{R}_{\text {Bonds }}^{\gamma}\right)^{\text {optimal }}}\right]^{\frac{1}{\gamma}} ; \\
& \theta=\left[\frac{E\left(\widetilde{R}_{\text {Bonds and Stocks }}^{\gamma}\right)^{\text {optimal }}}{E\left(\widetilde{R}_{\text {Stocks }}^{\gamma}\right)^{\text {optimal }}}\right]^{\frac{1}{\gamma}} .
\end{aligned}
$$

The denominator expectations are taken over the distributions implied by the relevant 3variable VAR, so I am calculating only the cost of investing in two stock or two bond indices rather than in four indices, and not the cost of using a restricted VAR for the portfolio formation.

And the proportionate opportunity cost, $\theta-1.0$, will be calculated for both the "only stocks" restriction and the "only bonds" restriction.

The above exercise is done for each of 11 alternative values of the risk aversion parameter, $\gamma$.

\subsection{Results}

Table 11 reports the results from calculating the proportionate opportunity cost for 11 different values of relative risk aversion for the two types of constrained portfolios: 


\section{Table 11}

The proportionate opportunity costs, $(\theta-1)$, optimal unconstrained portfolio weights and optimal ratios of stock indices to bond indices for various values of relative risk

aversion

\begin{tabular}{|c|c|c|c|c|c|}
\hline $\begin{array}{c}\text { Relative Risk } \\
\text { Aversion, (1- } \gamma \text { ) }\end{array}$ & Opportunity cost of investing & \multicolumn{2}{|c|}{ Optimal } & portfolio weights & Optimal ratios of \\
in stocks only & in bonds only & of stocks & of bonds & stocks to bonds \\
\hline 0.7 & 0.013 & 0.016 & 7.232 & 3.409 & 2.148 \\
1 & 0.009 & 0.014 & 6.948 & 2.850 & 2.439 \\
2 & 0.006 & 0.009 & 3.724 & 1.486 & 2.506 \\
3 & 0.004 & 0.005 & 2.577 & 1.002 & 2.572 \\
9 & 0.001 & 0.002 & 0.867 & 0.336 & 2.580 \\
10 & 0.001 & 0.002 & 0.785 & 0.304 & 2.582 \\
11 & 0.001 & 0.002 & 0.714 & 0.276 & 2.587 \\
12 & 0.001 & 0.002 & 0.656 & 0.253 & 2.593 \\
29 & 0.000 & 0.000 & 0.272 & 0.103 & 2.641 \\
30 & 0.000 & 0.000 & 0.266 & 0.100 & 2.660 \\
31 & 0.000 & 0.000 & 0.258 & 0.097 & 2.660 \\
\hline
\end{tabular}

${ }^{I}$ These two columns give the sum of shares placed in, respectively, the two stock indices or the two bond indices, by unconstrained investors.

portfolios that consist of two stock indices and Treasury bills, the "only stocks" portfolios, and portfolios that consist of two bond indices and Treasury bills, the "only bonds" portfolios, based on historically occurring asset returns over the ten-year period January 1992 through December 2001.

Of all the values of relative risk aversion examined the lowest proportionate opportunity cost of investing in "only stocks" portfolios, $0.00 \%(0.000)$, corresponds to the three highest levels of relative risk aversion of 29,30 , and 31 . This means that an investor with the level of risk aversion of 29 and higher being unconstrained will be equally happy as if he was constrained. The highest proportionate opportunity cost, $1.3 \%$ (0.013), corresponds to the lowest level of relative risk aversion of 0.7 and will be incurred by investors of that level of risk aversion should they decide to invest in "only 
stocks" portfolios rather than investing in both bonds and stocks. This means that an investor with the level of risk aversion of 0.7 being unconstrained will be equally happy as if he was constrained to stocks only but had $1.3 \%$ more initial wealth.

The lowest proportionate opportunity cost of investing in "only bonds" portfolios, $0.00 \%(0.000)$, again corresponds to the three highest levels of relative risk aversion of 29,30 , and 31 . In this case, the same as with "only stocks" portfolios, an investor with the level of risk aversion of 29 and higher being unconstrained will be equally happy as if he was constrained and had only bonds his portfolio. The highest proportionate opportunity cost, $1.6 \%(0.016)$, corresponds to the lowest level of relative risk aversion of 0.7 and will be incurred by investors of that level of risk aversion should they decide to invest in "only bonds" portfolios rather than in portfolios with both bonds and stocks. This means that an investor with the level of risk aversion of 0.7 being unconstrained will be equally happy as if he was constrained to bonds only but had $1.6 \%$ more initial wealth.

Table 11 clearly shows that as level of relative risk aversion increases, both proportionate opportunity costs of investing in "stocks only" and "bonds only" portfolios decrease, given the CRRA utility function (1).

These results suggest that optimal unconstrained portfolios offer high risktolerance investors broader, more daring investment opportunities than constrained optimal portfolios, and so the investors will require a premium to give up those investment opportunities.

What differs between the values of the proportionate opportunity costs for the two types of constrained optimal portfolios is the magnitude of the cost. For the low levels of risk aversion, from 0.7 to 3 , the values of the proportionate opportunity costs of investing 
in "only stocks" portfolios are lower than those for constrained optimal portfolios of "only bonds". It is reasonable to assume that high risk-tolerance investors, if they are to invest in either constrained portfolios, will prefer "only stocks" portfolio. This conclusion comes from observing the optimal unconstrained portfolio shares for stocks and bonds in Table 11 for high risk-tolerance investors. These investors follow very aggressive shortsell strategies in terms of Treasury bills by placing large proportions, larger than for bond indices, of their initial wealth in stock indices. Even though the constrained "only stocks" portfolios offer higher risk than constrained "only bonds" portfolio, they also offer higher expected returns than constrained "only bonds" portfolios (see Table 12). Therefore, if high risk-tolerance investors were forced to invest in "only bonds" portfolios the opportunity cost they incur under that strategy will be higher than that under "only stocks" strategy.

What is also interesting is the fact that for investors with risk aversion of nine and higher the values of the proportionate opportunity costs of investing in "only stocks" or in "only bonds" are the same. As investors become less and less risk-tolerant they place bigger and bigger proportions of initial wealth into Treasury bills. So, as risk aversion increases, investors if they were forced to invest in stocks and Treasury bills would place a bigger fraction of their initial wealth into Treasury bills and a smaller fraction of initial wealth into stock indices. The same happens to investors with portfolios of bond indices and Treasury bills. This change in portfolio weights makes stocks-and-Treasury-bills portfolios look like bonds-and-Treasury-bills portfolios: portfolios with a large amount of initial wealth placed into Treasury bills, an equivalent of cash, and with a smaller amount of initial wealth placed into risky assets (either bonds or stocks). Therefore, investors 
with risk aversion of nine and higher, by choosing either constrained portfolio, will incur the proportionate opportunity cost of the same magnitude.

The last column of Table 11 reports the ratios of optimal unconstrained portfolio shares of stocks to bonds. It is clear from the table that as relative risk aversion increases the optimal ratio of stocks to bonds slightly increases, but virtually stays constant. These results (slight increase in the optimal ratios) support Canner, Mankiw and Weil's (1997) and can be explained by the following fact. As investors become less and less risk tolerant, the portion of initial wealth placed into Treasury bills increases (see Table 12). At the same time portions of initial wealth they place into bonds and stock will decrease too but not at the same rate. Given that returns on Treasury bills and bonds are highly correlated, according to Canner, Mankiw and Weil (1997), highly risk-averse investors will reduce the proportion of their initial wealth they place into bonds at a higher rate than that they place into stocks. Therefore, as risk aversion increases, the optimal ratio of stocks to bonds will increase too. But if we consider the optimal ratios from Table 6 as virtually constant, then these results are consistent with the mutual-fund separation theorem according to which the ratio of stocks to bonds is constant for investors with different levels of risk aversion. But in both cases (the optimal ratios are slightly increasing or virtually constant) these results are not consistent with the popular advice according to which more risk-averse investors should hold a lower ratio of stocks to bonds.

Table 12 reports optimal portfolio shares for unconstrained and constrained portfolio strategies for three different levels of relative risk aversion: low (0.7), medium (11) and high (31). 
For optimal constrained and unconstrained portfolios for risk aversion of 0.7 more than $100 \%$ of initial wealth, $w_{0}$, is held in the nominally risky assets, stock and bond indices, and Treasury bills are held in negative quantities.

As risk aversion increases the proportion of initial wealth held in Treasury bills becomes positive and increases for optimal unconstrained and constrained portfolios, and correspondingly the proportion of initial wealth held in bond indices and stock indices decreases.

The table shows that unconstrained (and constrained) optimal portfolio shares are not similar for different levels of risk aversion. As a matter of fact, optimal unconstrained and constrained portfolios for the low level of relative risk aversion of 0.7 have more extreme quantities (negative as well as positive) of assets than optimal unconstrained and constrained portfolios for the medium level of relative risk aversion of 11 and for the high level of relative risk aversion of 31. Extremely negative quantities of assets for high risk-tolerance investors mean that the investors follow an aggressive short sale strategy.

Also Table 12 shows gross monthly expected returns on unconstrained and constrained optimal portfolios, $E\left(X^{* \prime} \widetilde{R}\right)$, for the three levels of relative risk aversion $(0.7,11$ and 31$)$. The net expected monthly portfolio return (gross expected monthly portfolio return minus 1.0 , multiplied by $100 \%$ ) for risk aversion of 0.7 is very dramatic for the unconstrained optimal portfolio, 5.9\%, and large for both constrained optimal portfolios $(3.7 \%$ for "only stocks" and $2.6 \%$ for "only bonds" portfolios). Net expected returns are of small size for risk aversion of 11 and of 31. Such extreme magnitudes of expected portfolio returns for high risk-tolerance investors confirm the previously made 
Table 12

Optimal portfolio shares for unconstrained and constrained to include stocks only or bonds only portfolios for different values of relative risk aversion

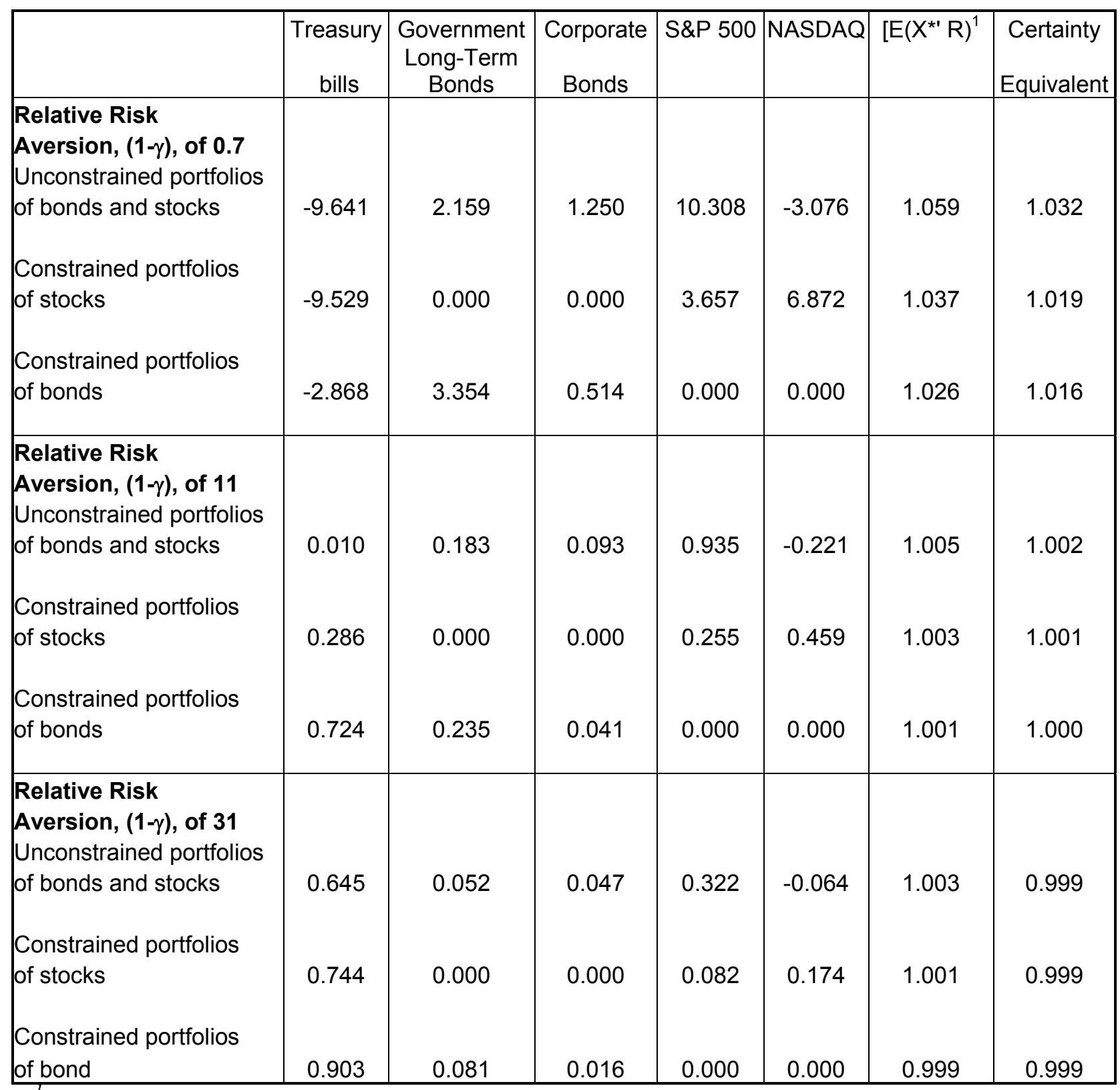

${ }^{\prime}$ Monthly gross expected return on portfolios. 
conclusion about very aggressive short sale strategies. These magnitudes represent very leveraged portfolios (unconstrained as well as constrained). For investors with risk aversion of 11 and 31 there is some short selling is going on too (in terms of NASDAQ index and only for the unconstrained portfolios), but not as aggressive as for investors with risk aversion of 0.7 . The less aggressive short selling for medium or high risk aversion leads to lower mean return portfolios.

In comparing unconstrained expected portfolio returns and constrained expected portfolio returns for the three levels of risk aversion I find that unconstrained and constrained expected portfolio returns for risk aversion of 11 and of 31 are closer to each other than that for risk aversion of 0.7. This shows that as risk aversion increases the more nearly indifferent an investor is between the unconstrained and constrained portfolio strategies.

It is also interesting to compare expected monthly portfolio returns from Table 7 and the values of the proportionate opportunity cost reported in Table 6. For risk aversion of 0.7 the proportionate opportunity cost of investing in the "only stocks" portfolio reaches $1.3 \%$ and the proportionate opportunity cost of investing in the "only bonds" portfolio reaches $1.6 \%$; unconstrained investors have the net expected monthly portfolio return (gross expected portfolio return minus 1.0, multiplied by $100 \%$ ) of $5.9 \%$. For risk aversion of 11 the proportionate opportunity cost of investing in either of two constrained portfolios is $0.1 \%$ for stocks and $0.2 \%$ for bonds, and the net expected monthly portfolio return for unconstrained investors is $0.5 \%$. For the level of risk aversion of 31 the proportionate opportunity cost of investing in a constrained portfolio is $0.0 \%$. 
Table 12 also reports the certainty equivalents calculated for the same three levels of relative risk aversion $(0.7,11$ and 31$)$. The certainty equivalent, $(C E)$, is defined by

$$
\frac{1}{\gamma} C E^{\gamma}=\frac{1}{\gamma} w_{0}^{\gamma} E\left(\widetilde{R}^{\gamma}\right)
$$

and so, with $w_{0}=1$,

$$
C E=\left(E\left[\widetilde{R}^{\gamma}\right]\right)^{\frac{1}{\gamma}} .
$$

The certainty equivalent represents the amount of certain wealth that would be viewed with indifference to the optimal portfolio. It is computed for investors of different levels of risk aversion: low (of 0.7), medium (of 11) and high (of 31). The table shows that as risk aversion increases the value of the certainty equivalent decreases (for the unconstrained portfolio strategy as well as for the constrained). This suggests that as investors become more afraid of risk they use less risky portfolio strategies and will be expecting lower returns from those portfolios and, so, the certain amount of wealth they will be willing to accept with indifference will decrease.

It is interesting to compare certainty equivalents for unconstrained optimal portfolio strategies from Table 12 and the values of the proportionate opportunity cost from Table 11. For the level of risk aversion of 0.7 the proportionate opportunity cost of investing in the "only stocks" portfolio is $1.3 \%$ while the unconstrained net certainty equivalent (certainty equivalent minus 1.0 ) reaches $3.2 \%$; the proportionate opportunity cost of investing in the "only bonds" portfolio is $1.6 \%$. As the level of risk aversion increases to 31 the proportionate opportunity cost for both types constrained portfolios falls to $0.0 \%$ and the unconstrained net certainty equivalent falls to $0.0 \%$. 
Table 13 presents the percentage of gross certainty equivalent for unconstrained portfolio strategies lost due to the constraint of investing in "only stocks" portfolios and to the constraint of investing in "only bonds" portfolios, computed as shown in (27). The percentage of gross certainty equivalent lost due to the constraints is timeless just like the proportionate opportunity cost, $\theta-1.0$.

$$
\text { The percentage loss }=\frac{C E^{\text {Uncd }}-C E^{\text {Const }}}{C E^{\text {Uncd }}} * 100 \%=\frac{\theta-1.0}{\theta} * 100 \%
$$

The highest percentage loss, $1.6 \%$, happens for the investors with risk aversion of 0.7 holding "only bonds" in their portfolios. As risk aversion increases, for both "only stocks" and "only bonds" portfolios, the percentage loss decreases. The lowest percentage loss, $0.0 \%$, is observed for the investors with risk aversion of 29 and higher holding either "only stocks" or "only bonds" in their portfolios. Table 13 confirms the previously made conclusion that as investors become more afraid of risk their perceptions of the optimal constrained and optimal unconstrained portfolio strategies become more and more similar due to investing a large portion of their initial wealth into Treasury bills.

Table 13

The percentage of certainty equivalent lost due to the "only stocks" and "only bonds" constraints for various levels of relative risk aversion

\begin{tabular}{|c|c|c|c|c|c|c|c|c|c|c|c|c|}
\hline Type of & \multicolumn{10}{c|}{ Relative } & Risk & Aversion, \\
\cline { 2 - 11 } \\
\cline { 2 - 12 }
\end{tabular}

The percentage loss $=\frac{C E^{\text {Uncd }}-C E^{\text {Cond }}}{C E^{\text {Uncd }}} * 100 \%=\frac{\theta-1}{\theta} * 100 \%$. 
Hence, as the level of risk aversion increases the certain amount of wealth unconstrained investors and constrained investors will be willing to accept with indifference will be getting closer to each other, and, therefore, the percentage loss in certainty equivalents due to the constraint will decrease.

\subsection{Conclusion}

In this part of the second essay I have investigated the opportunity cost incurred by investors when they are constrained to use only one type of assets, either stocks or bonds, instead of being unconstrained and using both stocks and bonds. The original historical asset returns are used along with CRRA utility functions, and the proportionate opportunity cost. The opportunity cost has been calculated for different values of relative risk aversion (including extreme levels of relative risk aversion) for "only stocks" and "only bonds" portfolios. The highest proportionate opportunity cost found is $1.6 \%$ (0.016) for the level of relative risk aversion of 0.7 for "only bonds" portfolios. The lowest proportionate opportunity cost found is $0.0 \%(0.000)$ for the level of relative risk aversion of 29 and higher for both types of constrained portfolio strategies. I found for both types of constrained optimal portfolios that as the level of relative risk aversion increases the proportionate opportunity cost decreases.

The only difference between estimates of the proportionate opportunity cost for the two constrained portfolios is the magnitude of the estimates. They are bigger for the level of risk aversion of 0.7 for the "only bonds" portfolios. This can be explained by the fact that high risk-tolerance investors prefer "only stocks" portfolios to "only bonds" portfolios and will be more satisfied with "only stocks" portfolios that offer higher risk 
and higher returns than with "only bonds" portfolios. Therefore, the investors will require a lower proportion of initial wealth while being constrained by "only stocks" than by "only bonds". However, the difference in these opportunity costs is slight.

My findings of optimal ratios of stocks to bonds for different levels of risk aversion confirm the mutual-fund separation theorem and contradict popular financial advice. I found that as the level of risk aversion increases the optimal ratio of stocks to bonds virtually stays constant.

Based on my calculations, I may conclude that for investors with high levels of relative risk aversion (nine and above), constrained portfolios that include only one type of assets, stocks only or bonds only, along with Treasury bills perform as well as unconstrained portfolios that include both types of assets, stocks and bonds. 


\section{References:}

Brennan, M.J., W.N.Torous, Individual Decision-Making and Investor Welfare, Economic Notes, 1999, 28(2), 119-43

Campbell, John Y., Viceira, Luis H., Strategic Asset Allocation, Oxford University Press, March, 2002

Canner N., N.G. Mankiw, D.N. Weil, An Asset Allocation Puzzle, The American Economic Review, March 1997, 181-190

Cheng, Ping; Liang, Youguo, Optimal Diversification: Is It Really Worthwhile? Journal of Real Estate Portfolio Mangement, 2000, 6(1), 7-106

Fama, E. F., Foundation of Finance, Basic Books, Inc., Publishers, New York, 1972, 25256

Haliassos, M., C.C. Bertaut, Why do so Few Hold Stocks? The Economic Journal, 1995,105 (432), 1110-29

Ibbotson Associates, SBBI, Stocks, Bonds, Bills, and Inflation ${ }^{\circledR} 2002$ Yearbook

Kelly, Morgan, All Their Eggs in One Basket: Portfolio Diversification of US Households, Journal of Economic Behavior and Organization, June 1995, 27(1), 8796

Kroll, Yoram, Haim Levy and Harry M. Markowitz, Mean-Variance Versus Direct Utility Maximization, The Journal of Finance, 1984, 1851-72

Sankaran, Jayaram K., Patil, Ajay A., On the Optimal Selection of Portfolios under Limited Diversification, Journal of Banking and Finance, November 1999, 23(11), $1655-66$ 
Simaan, Yusif, What Is the Opportunity Cost of Mean-Variance Investment Strategy? Management Science, 1993, 39(5), 578-87

Vassal, Valdimir de, Risk Diversification Benefits of Multiple-Stock Portfolios, The Journal of Portfolio Management, Winter 2001, 32-39 


\title{
Chapter 4. Third Essay:
}

\section{The Opportunity Cost for an Investor of Being Restricted from}

\section{Short-Selling}

\begin{abstract}
Short-selling is a legitimate trading strategy. A restriction on short-selling will reduce investment opportunities for an investor, and will create a sub-optimal asset allocation that results in a welfare loss for the investor. To measure that welfare loss I compare optimal portfolios that allow short-selling with optimal portfolios that do not allow short-selling by using the concept of the proportionate opportunity cost along with various CRRA utility functions. Two sets of asset returns are used with a VAR in generating joint returns distributions for the portfolio formation period: the original historical asset returns data set, and the historical asset returns with extreme values exaggerated. In each case 1,000 alternative sets of from three to 24 assets including one with a risk-free nominal return are randomly made available for investment. I show that the optimal portfolio strategy with the short-selling constraint performs almost as well as the unconstrained portfolio strategy for investors with medium levels of risk aversion, and performs as well as the unconstrained portfolio strategy for investors with high levels of risk aversion. The results, derived from the original historical asset returns data set with no exaggeration of extreme returns, show that investors' welfare losses reach $12.8 \%$ of initial wealth for investors with low risk aversion. With extreme returns exaggerated in the returns distribution, investors' welfare losses reach $13.5 \%$ of initial wealth. The results in both cases indicate that less risk-averse investors experience greater welfare losses, and that the short-selling constraint reduces the cost of sub-optimal diversification.
\end{abstract}




\section{Chapter 4. Third Essay:}

\section{The Opportunity Cost for an Investor of Being Restricted from \\ Short-Selling}

\section{Introduction}

Short-selling is a legitimate trading strategy. By short-selling an investor will get extra money to invest and will make positive returns if the shorted assets' price rises by less than that of the assets in which the extra funds are placed. At the same time it is a risky strategy because the investor will lose money if the price of the shorted asset rises by more than that of the assets in which the extra funds are placed. An optimal portfolio may require an investor to hold extremely long or extremely short investment positions. These extreme long or short holding positions sometimes are difficult to implement in practice because investors face constraints on their portfolio holdings. For example, Regulation T, which applies to almost all investors, institutional as well as individual, requires $50 \%$ margin.

Any short-selling restriction will reduce investment opportunities for an investor, and will create a constrained portfolio strategy. Therefore, the question that I am interested in and will work with in this essay is: how bad it is for an investor to be restricted from short-selling? Or, what would one's welfare loss be from being faced with a short-selling constraint.

The introduction to this dissertation showed that the proportionate opportunity cost is the best way to measure investors' welfare losses. Under the assumption of the constant relative risk aversion utility function 


$$
\mathrm{U}(\widetilde{w})=\left\{\begin{array}{l}
\frac{1}{\gamma} \widetilde{w}^{\gamma}, \gamma<1, \gamma \neq 0, \widetilde{w}>0 \\
-\infty, \widetilde{w} \leq 0
\end{array}\right.
$$

the proportionate opportunity cost (willingness to accept payment as compensation for being constrained) can be calculated as $\theta-1.0$ where $\theta$ is defined by

$$
\mathrm{EU}\left(\theta w_{0} \widetilde{R}^{c}\right)=\mathrm{EU}\left(w_{0} \widetilde{R}^{u}\right)
$$

where $w_{0}$ is the initial wealth, and $\widetilde{R}^{u}$ and $\widetilde{R}^{c}$ are the stochastic returns per dollar invested in the unconstrained and constrained portfolios. Solving (2) gives

$$
\theta=\left[\frac{E\left(\widetilde{R}^{\gamma}\right)^{\text {unconstrained }}}{E\left(\widetilde{R}^{\gamma}\right)^{\text {constrained }}}\right]^{\frac{1}{\gamma}} .
$$

Under CRRA, (1), one plus the proportionate willingness to accept payment and the ratio of certainty equivalents of unconstrained to constrained optimal portfolios are equal, as shown in the introduction of this dissertation. Since the ratio of certainty equivalents is unitless and in particular has no time units, the proportionate opportunity cost, $\theta-1.0$, is timeless. But its numerical value depends on a number of months until horizon, i.e. with the investment horizon of $T$ months the proportionate willingness to accept payment is $\theta^{T}$.

There are several articles that address the issue of the cost of placing restrictions on short-selling that I would like to discuss first.

Tepla (2000) used mean-variance portfolios and worked in a dynamic environment. She found that short-sale restrictions create inefficient portfolios, and being restricted from short-selling, “...[i]nvestors act as if unconstrained when investing only in a subset of the risky assets". She found that the opportunity cost in her case is the reduced 
number of assets to invest in. But reducing the number of assets in one's portfolio might hurt the diversification aspect.

The use of the mean-variance framework in Tepla's work is not appealing. In her study investors are being constrained, first, by the mean variance framework, and, second, by the short-selling constraint. The best way to approach the problem of calculating the opportunity cost of short-selling restrictions, which I will follow here, is to use optimal portfolios (not mean-variance efficient as Tepla did, but rather globally optimal) that can be found through an optimization procedure.

Wang (1998) dealt with the mean-variance framework as well, and worked with one-period model. He used annualized monthly returns on 26 stock portfolios and constructed posterior distributions for those portfolios. Wang worked with two types of short-selling constraints: (1) no short position of an asset is allowed to be greater than $50 \%$ of initial wealth; and (2) all short positions are prohibited. In his calculations Wang found the same thing as Tepla did: short-sale restrictions do create inefficient portfolios. The opportunity cost that Wang found is the loss in expected portfolio return that comes from "...[h]olding the given portfolio instead of an efficient portfolio with the same variance". And as a short-selling constraint becomes more restrictive the loss in the expected portfolio return increases. As I have explained earlier, the use of the meanvariance framework is not appealing in calculating the opportunity cost of a short-selling constraint. The best way, which I will follow here, to measure the cost is to compare the constrained well-diversified optimal portfolios that do not allow short-selling and unconstrained well-diversified optimal portfolios that can be found through an optimization procedure. 
Best and Grauer (1991) also worked with the mean-variance framework. The question they asked was: how would a change in the mean return of a security in an investor's portfolio affect the portfolio return. While answering the question they found out also that a non-negativity constraint on portfolio holdings results in a lower (by at least one security and by a maximum of one half of the portfolio's securities) number of assets in the portfolio. So, an effect of a short-selling constraint in their case was, as it was in Tepla's, a reduced number of assets chosen to invest in.

Luttmer (1996) pointed out that short-selling constraints on Treasury bills prevent investors from exploiting the equity premium by borrowing at the Treasury bill rate and investing in the stock market. So, the cost of a short-selling constraint in his case, as it was in Wang's, is the lower expected portfolio return.

These articles answer the following questions: how will a short-selling restriction affect an investor's portfolio return, and the number of assets in the portfolio? But the question that remains unanswered and the question I ask in this essay is how a shortselling restriction affects an investor's welfare. The question is answered by calculating the proportionate opportunity cost for an investor of being restricted from short-selling.

The procedure followed in this essay for calculating this proportionate opportunity cost includes random asset selection for investors' portfolios, estimation of a vector autoregressive process, derivation of the joint probability distribution function of asset returns, and computing optimal constrained and unconstrained portfolios.

In this essay I show that with a nominally risk-free asset the optimal portfolio strategy with the short-selling constraint performs almost as well as the unconstrained portfolio strategy for investors with medium levels of risk aversion, and performs as well 
as the unconstrained portfolio strategy for investors with high levels of risk aversion. The results, derived from the original historical asset returns data set with no exaggeration of extreme returns, show that investors' welfare losses reach $12.8 \%$ of initial wealth for investors with low risk aversion; and become even larger when extreme returns in the original historical data set are exaggerated.

The second section of this essay describes the procedure of random asset selection for investors' portfolios, of inferring the joint probability distribution function of asset returns, of computing constrained and unconstrained optimal portfolios, and of calculating of the proportionate opportunity cost. The third section discusses the results of the study, and the fourth section concludes.

\section{The Procedure}

\subsection{Asset Selection}

The procedure of calculating the proportionate opportunity cost for different levels of risk aversion will be performed 1,000 times in each case using a well-diversified optimal portfolio with $n-1$ randomly picked nominally risky assets and Treasury bills as the nominally risk-free asset. The well-diversified number of assets, $n$, for an optimal portfolio for every level of risk aversion comes from the previous essay. I defined the well-diversified number of assets the following way. At a certain $n$ I found that further diversification is no longer very helpful: the opportunity cost of investing in these $n$ assets rather than in a larger number of assets (in the second essay this "larger number of assets" was 26 as an approximation of infinity) does not exceed $1 \%$ of initial wealth. I found that for investors with relative risk aversion of respectively $0.7,1,2,3,9,10,11$, 
12,29 or higher the well-diversified number of assets is $24,23,22,20$, nine, eight, seven, six, and three or less. In the case with the well-diversified number of assets the proportionate opportunity cost of a short-selling constraint is directly affected by the level of relative risk aversion, and also indirectly affected because different levels of relative risk aversion require different number of assets for good diversification.

Alternatively, the procedure of calculating the proportionate opportunity cost of the short-selling constraint for different levels of risk aversion will be repeated 1,000 times for ten assets as well: nine randomly picked nominally risky assets and Treasury bills. I will do that for ten assets (the number is suggested by the literature on constrained portfolio strategies, e.g. Tew, Reid and Witt (1991)) to see how the proportionate opportunity cost of a short-selling restriction changes with the change in the level of risk aversion only.

In the process of calculating the proportionate opportunity cost and discussing the results of my calculations I will refer to the chosen $n$ as the "well-diversified number of assets". I will be comparing results for the "well-diversified number of assets" and for ten assets. For investors with risk aversion of 9 and higher the well-diversified number of assets is less then ten. But still those $n$-asset portfolios, and not ten-asset portfolios, will be well-diversified by the definition I gave earlier.

The first step in the procedure of calculating the proportionate opportunity cost of a short-selling restriction is to pick at random $n-1$ nominally risky assets, as the welldiversified number of nominally risky assets. Then, to construct the optimal constrained and optimal unconstrained portfolios I need to get expected values of real returns for the $n$ assets I am using for time $T+1$ : for the $n-1$ nominally risky assets and for nominally 
risk-free Treasury bills. In real terms, though, there is no risk-free asset. Returns on Treasury bills are risk-free only in nominal terms. But in time-series data inflation will be uncertain in any period and, thus, so will the real rate of return on Treasury bills. Therefore, the $n$ assets that I am dealing with in real terms will all be risky assets. The same procedures are also conducted with ten assets instead of $n$.

\subsection{Vector Autoregressions of Returns}

So, to get expected values of real returns for the case of $n$ assets at time $T+1$, the portfolio formation period, I estimate a vector autoregressive process (VAR). The next steps are to derive the joint probability distribution for the $n$ assets' real returns, and, finally, to construct optimal constrained and optimal unconstrained portfolios.

To derive the joint probability distribution of empirical deviations from the VARestimated conditional means for those randomly picked asset returns I do the following.

The nominal return on asset $i$ at time $t$ minus the nominal return on Treasury bills at time $t$ gives me the excess return on asset $i\left(x_{i, t}\right)$ at time $t$ for $i=1, \ldots, n-1$ and for $t=1$, $\ldots, T$. When I run a VAR for excess returns of those $n-1$ assets and realized inflation, as

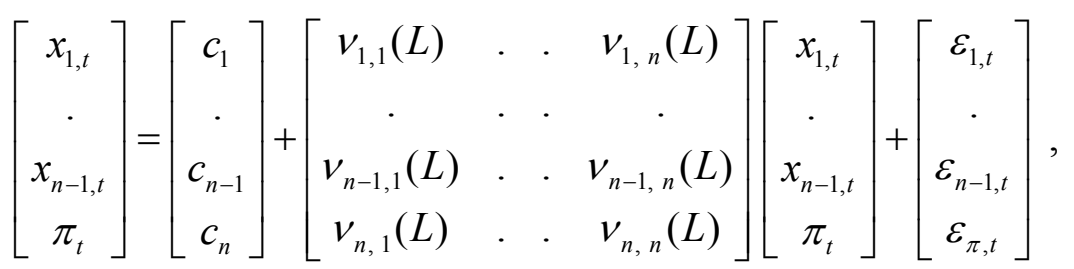

I obtain $\left\{\hat{c}_{i}\right\},\left\{\hat{\varepsilon}_{i, t}\right\}$ and $\left\{\hat{v}_{i, k}(L)\right\}$, where

$$
\hat{v}_{i, k}(L)=\hat{\delta}_{i, k}^{1} L^{1}+\hat{\delta}_{i, k}^{2} L^{2}+\ldots
$$

Then, I compute the vector of conditional expected values of excess returns for time $T+1$ and expected inflation for time $T+1$ as: 


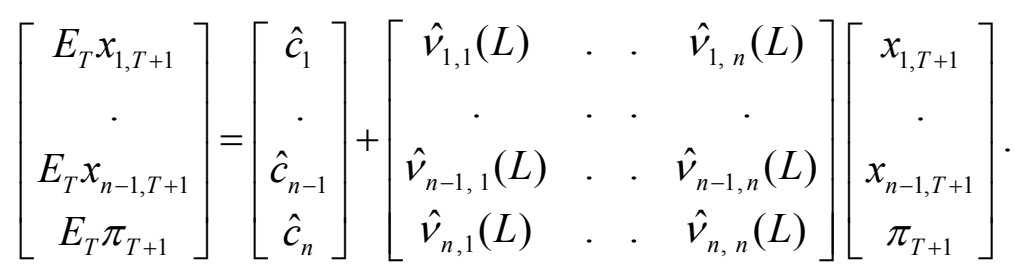

Next, the expected real return on asset $i$ in period $T+1$, the portfolio formation period, is

$$
\left[\begin{array}{c}
E_{T} r_{1, T+1} \\
\cdot \\
E_{T} r_{n-1, T+1}
\end{array}\right]=\left[\begin{array}{c}
E_{T} x_{1, T+1} \\
\cdot \\
E_{T} x_{n-1, T+1}
\end{array}\right]+\left[\begin{array}{c}
r_{T B, T+1}^{n} \\
\cdot \\
r_{T B, T+1}^{n}
\end{array}\right]-\left[\begin{array}{c}
E_{T} \pi_{T+1} \\
\cdot \\
E_{T} \pi_{T+1}
\end{array}\right]
$$

where $r_{T B, T+1}^{n}$ is the ex ante observed nominal return on Treasury bills for time $T+1$. The expected real return on Treasury bills for time $T+1$ is

$$
E_{T} r_{T B, T+1}=r_{T B, T+1}^{n}-E_{T} \pi_{T+1} .
$$

Finally, the conditional probability distribution for real returns for time $T+1$ is determined by

$$
\left[\begin{array}{c}
\widetilde{r}_{1, T+1} \\
\cdot \\
\widetilde{r}_{n-1, T+1} \\
\widetilde{r}_{T B, T+1}
\end{array}\right]=\left[\begin{array}{c}
E_{T} x_{1, T+1} \\
\cdot \\
E_{T} x_{n-1, T+1} \\
0
\end{array}\right]+\left[\begin{array}{c}
r_{T B, T+1}^{n} \\
\cdot \\
r_{T B, T+1}^{n} \\
r_{T B, T+1}^{n}
\end{array}\right]-\left[\begin{array}{c}
E_{T} \pi_{T+1} \\
\cdot \\
E_{T} \pi_{T+1} \\
E_{T} \pi_{T+1}
\end{array}\right]+\left[\begin{array}{c}
\widetilde{\varepsilon}_{1, T+1} \\
\cdot \\
\widetilde{\varepsilon}_{n-1, T+1} \\
0
\end{array}\right]-\left[\begin{array}{c}
\widetilde{\varepsilon}_{\pi, T+1} \\
\cdot \\
\widetilde{\varepsilon}_{\pi, T+1} \\
\widetilde{\varepsilon}_{\pi, T+1}
\end{array}\right]
$$

where $\left[\begin{array}{c}\widetilde{\varepsilon}_{1, T+1} \\ \cdot \\ \widetilde{\varepsilon}_{n-1, T+1} \\ \widetilde{\varepsilon}_{\pi, T+1}\end{array}\right]$ takes on the historically observed values $\left[\begin{array}{c}\widetilde{\varepsilon}_{1, t} \\ \cdot \\ \widetilde{\varepsilon}_{n-1, t} \\ \widetilde{\varepsilon}_{\pi, t}\end{array}\right]$ from regression (4), $t=1,2, \ldots, T$, with equal probabilities $(1 / T)$.

This way of deriving asset returns probability distribution functions, using historically occurring innovations to asset returns captured through this VAR procedure, is superior to the VAR methods mentioned in the literature, e.g. Campbell and Viceira (2002). The literature on derivation of asset returns probability distribution functions 
assumes that the distribution of asset returns is static, not evolving over time. But the reality is such that the asset returns distribution is dynamic, depending on both recent realizations and the fixed historical distribution of shocks to the dynamic asset returns process. So the right way of deriving asset returns probability distribution functions is to include the dynamics of the past history of asset returns.

\subsection{Constrained Portfolios}

Regulation $\mathrm{T}$ imposes on all investors, individual as well as institutional, 50\% margin requirements. These requirements say that the ratio between the total position size (the sum of absolute values of portfolio holdings for all nominally risky assets) and initial wealth cannot be greater than two. This $50 \%$ margin restriction is implemented in the essay the following way.

Using the information about those randomly picked assets' derived probability distributions for their real returns (computed as shown in (9)), I compute the constrained optimal portfolio: the solution of

$$
\underset{\left\{\alpha_{1}, \ldots, \alpha_{n-1}\right\}}{\operatorname{Max}} E U(\widetilde{w})=\operatorname{Max} E\left\{\frac{1}{\gamma}\left[w_{0}\left(\alpha_{1} \widetilde{r}_{1}+\ldots+\alpha_{n-1} \widetilde{r}_{n-1}+\left(1-\alpha_{1}-\ldots-\alpha_{n-1}\right) \widetilde{r}_{T B}\right)\right]^{\gamma}\right\}
$$

subject to the short-selling constraint:

$$
\sum_{i=1}^{n-1}\left|\alpha_{i}\right| \leq 2 w_{0}
$$

where $w_{0}$ is initial wealth, set equal to $1 ; \alpha_{1}, \ldots, \alpha_{n-1}$ are the first $n-1$ individual assets' portfolio shares in the constrained optimal portfolio. To get the portfolio I search over $\alpha_{1}, \ldots, \alpha_{n-1}$ space to optimize expected utility, using nonlinear optimization by a quasiNewton method based on convergence to first-order conditions of problem (10). Again, 
the expectation is taken over the joint probability distribution derived as described above in (4)-(9).

\subsection{Unconstrained Portfolios}

The next step, then, is to get the unconstrained optimal portfolio: the solution of

$$
\underset{\left\{\beta_{1}, \ldots, \beta_{n-1}\right\}}{\operatorname{Max}} E U(\widetilde{w})=\operatorname{Max} E\left\{\frac{1}{\gamma}\left[w_{0}\left(\beta_{1} \widetilde{r}_{1}+\ldots+\beta_{n-1} \widetilde{r}_{n-1}+\left(1-\beta_{1}-\ldots-\beta_{n-1}\right) \widetilde{r}_{T B}\right)\right]^{\gamma}\right\}
$$

where $\beta_{1}, \ldots, \beta_{n-1}$ are the first $n-1$ individual assets portfolio shares in the unconstrained optimal portfolio. To get the portfolio I search over $\beta_{1}, \ldots, \beta_{n-1}$ space to optimize expected utility, using nonlinear optimization by a quasi-Newton method based on convergence to first-order conditions of problem (12). Again, the expectation is taken over the joint probability distribution derived as described above in (4)-(9).

\subsection{Calculating Opportunity Cost}

Now, when I have the constrained optimal and unconstrained optimal portfolios I calculate the opportunity cost, $\theta-1.0$. For the formula for $\theta$, (3), I need to find $\mathrm{E}\left(\widetilde{R}^{\gamma}\right)^{\text {unconstrained }}$ and $\mathrm{E}\left(\widetilde{R}^{\gamma}\right)^{\text {constrained }}$.

$\mathrm{E}\left(\widetilde{R}^{\gamma}\right)^{\text {unconstrained }}$ (referring more completely to $E_{T}\left(\widetilde{R}_{T+1}^{\gamma}\right)^{\text {unconstrained }}$ ) is equal to

(13) $E_{T}\left(\widetilde{R}_{T+1}^{\gamma}\right)^{\text {unconstrd }}=\frac{1}{T} \sum_{t=1}^{T}\left\{\left[\begin{array}{llll}\beta_{1}^{*} & \ldots & \beta_{n-1}^{*} & 1-\beta_{1}^{*}-\ldots-\beta_{n-1}^{*}\end{array}\right]\left[\begin{array}{c}E_{T} r_{1, T+1}+\varepsilon_{1, t}-\varepsilon_{\pi, t} \\ \cdot \\ E_{T} r_{n-1, T+1}+\varepsilon_{n-1, t}-\varepsilon_{\pi, t} \\ E_{T} r_{T B, T+1}+0-\varepsilon_{\pi, t}\end{array}\right]\right\}$ 
where the vector of $\beta_{i}^{*}$ is the vector of optimal unconstrained portfolio shares; the vector of $E_{T} r_{i, T+1}+\varepsilon_{i, t}-\varepsilon_{\pi, t}$ and $E_{T} r_{T B, T+1}-\varepsilon_{\pi, \mathrm{t}}$ are the vectors of particular possible values of real returns (conditional on the data set for times $t=1$ through $T$ ) at time $T+1$ (the portfolio formation period) and calculated as shown in (4)-(9).

And $\mathrm{E}\left(\widetilde{R}^{\gamma}\right)^{\text {constrained }}$ is equal to

(14) $E_{T}\left(\widetilde{R}_{T+1}^{\gamma}\right)^{\text {consted }}=\frac{1}{T} \sum_{t=1}^{T}\left\{\left[\begin{array}{llll}\alpha_{1}^{*} & \ldots & \alpha_{n-1}^{*} & 1-\alpha_{1}^{*}-\ldots-\alpha_{n-1}^{*}\end{array}\right]\left[\begin{array}{c}E_{T} r_{1, T+1}+\varepsilon_{1, t}-\varepsilon_{\pi, t} \\ \cdot \\ E_{T} r_{n-1, T+1}+\varepsilon_{n-1, t}-\varepsilon_{\pi, t} \\ E_{T} r_{T B, T+1}+0-\varepsilon_{\pi, t}\end{array}\right]\right\}^{\gamma}$

where $\alpha_{i}^{*}$ is the vector of optimal constrained portfolio shares.

Then, having calculated (13) and (14), I use (3) to get a numerical value for $\theta$.

The whole procedure, starting from picking $n-1$ nominally risky assets, is being repeated 1,000 times. This gives me 1,000 values of $\theta$. The procedure is done for each of 11 alternative values of the risk aversion parameter $\gamma$ each time for the well-diversified number of assets, $n$, and for ten assets.

\section{Results}

The results from this research project are as follows.

\subsection{Results Derived from Historical Returns Data with No Exaggeration of Extreme Returns.}

\subsubsection{Opportunity Costs}

Table 1 and Table 2 represent the results from calculation of 1,000 values of the proportionate opportunity cost for 11 different values of relative risk aversion for 
alternatively the well-diversified number of assets for each level of risk aversion and for ten assets, based on historically occurring asset returns over the 10-year period January 1992 through December 2001.

Both tables clearly show that as the level of relative risk aversion increases the proportionate opportunity cost decreases, given the CRRA utility function, (1). This is not surprising. As risk aversion decreases the investor considers the optimal unconstrained portfolio as his best choice that does not place any restrictions on his investment behavior and lets him follow a very aggressive short sale strategy that will not be possible under the constrained portfolio strategy (see Table 3 and Table 4 below), and, therefore, he will require a higher proportion of initial wealth as the payment to stay constrained and be indifferent to the optimal constrained portfolio with the short-selling restriction.

Table 1 and Table 2 also show that as the level of relative risk aversion increases the standard deviations of the proportionate opportunity costs decrease: the distributions of the opportunity cost are getting "tighter". This can be explained by the following: as risk aversion increases, first, investors become less and less engaged into short-selling due to a decrease in their risk-tolerance; therefore, at some point the short-selling constraint stops binding. Second, a bigger and bigger proportion of initial wealth will be placed by the investors into Treasury bills (see Table 3 and Table 4), the least shorted asset from their point of view, and a smaller and smaller proportion of initial wealth will be placed into nominally risky assets, the most shorted assets. The combination of these two facts results in portfolios that very much resemble each other. Thus we observe the low standard deviation of the proportionate opportunity cost. 


\section{Table 1}

The proportionate opportunity cost of the short-selling constraint, $(\theta-1)$, for various values of relative risk aversion for the well-diversified number of assets

\begin{tabular}{|c|c|c|c|c|c|c|}
\hline $\begin{array}{c}\text { Relative } \\
\text { Risk Aversion, (1- } \gamma \text { ) }\end{array}$ & $\begin{array}{c}\text { \# of } \\
\text { Assets }\end{array}$ & Smallest & Mean & Median & Largest & $\begin{array}{c}\text { Standard } \\
\text { Deviation }\end{array}$ \\
\hline Low & & & & & & \\
$\mathbf{0 . 7}$ & 24 & 0.043 & 0.128 & 0.121 & 0.583 & 0.046 \\
$\mathbf{1}$ & 23 & 0.022 & 0.086 & 0.074 & 0.556 & 0.032 \\
$\mathbf{2}$ & 22 & 0.007 & 0.068 & 0.055 & 0.486 & 0.013 \\
$\mathbf{3}$ & 20 & 0.001 & 0.040 & 0.029 & 0.444 & 0.006 \\
Medium & & & & & & \\
$\mathbf{9}$ & 9 & 0.000 & 0.010 & 0.000 & 0.193 & 0.004 \\
$\mathbf{1 0}$ & 8 & 0.000 & 0.008 & 0.000 & 0.172 & 0.004 \\
$\mathbf{1 1}$ & 7 & 0.000 & 0.004 & 0.000 & 0.154 & 0.003 \\
$\mathbf{1 2}$ & 6 & 0.000 & 0.003 & 0.000 & 0.148 & 0.003 \\
High & & & & & & \\
$\mathbf{2 9}$ & 3 & 0.000 & 0.000 & 0.000 & 0.003 & 0.001 \\
$\mathbf{3 0}$ & 3 & 0.000 & 0.000 & 0.000 & 0.001 & 0.001 \\
$\mathbf{3 1}$ & 3 & 0.000 & 0.000 & 0.000 & 0.001 & 0.001 \\
\hline
\end{tabular}

Table 2

The proportionate opportunity cost of the short-selling constraint, $(\theta-1)$, for various values of relative risk aversion for ten assets

\begin{tabular}{|c|c|c|c|c|c|}
\hline $\begin{array}{c}\text { Relative } \\
\text { Risk Aversion, (1- } \gamma)\end{array}$ & Smallest & Mean & Median & Largest & Standard Deviation \\
\hline \multicolumn{6}{|l|}{ Low } \\
\hline 0.7 & 0.003 & 0.080 & 0.061 & 0.498 & 0.045 \\
\hline 1 & 0.001 & 0.056 & 0.047 & 0.461 & 0.025 \\
\hline 2 & 0.001 & 0.033 & 0.030 & 0.413 & 0.019 \\
\hline $\begin{array}{c}3 \\
\text { Medium }\end{array}$ & 0.000 & 0.021 & 0.011 & 0.384 & 0.009 \\
\hline 9 & 0.000 & 0.006 & 0.000 & 0.185 & 0.006 \\
\hline 10 & 0.000 & 0.006 & 0.000 & 0.156 & 0.004 \\
\hline 11 & 0.000 & 0.005 & 0.000 & 0.126 & 0.003 \\
\hline 12 & 0.000 & 0.005 & 0.000 & 0.128 & 0.003 \\
\hline \multicolumn{6}{|l|}{ High } \\
\hline 29 & 0.000 & 0.001 & 0.000 & 0.004 & 0.001 \\
\hline 30 & 0.000 & 0.001 & 0.000 & 0.002 & 0.001 \\
\hline 31 & 0.000 & 0.000 & 0.000 & 0.002 & 0.001 \\
\hline
\end{tabular}


For the well-diversified number of assets, Table 1 shows that the lowest mean (over 1,000 replications) of the proportionate opportunity cost, $0.0 \%(0.000)$, corresponds to the level of risk aversion of 29 and higher. This means that an investor with the level of relative risk aversion of 29 and higher being unconstrained will be equally happy as if he was constrained. The highest mean (over 1,000 replications) of the proportionate opportunity cost, $12.8 \%(0.128)$, corresponds to the very low level of relative risk aversion of 0.7 . This means that an investor with the level of relative risk aversion of 0.7 being unconstrained will be equally happy as if he was constrained but had $12.8 \%$ more of initial wealth.

For low levels (from 3 to 0.7 ) of relative risk aversion the mean (over 1,000 replications) of the proportionate opportunity cost ranges from $4.0 \%(0.040)$ for the relative risk aversion of 3 to $12.8 \%(0.128)$ for the relative risk aversion of 0.7 .

For medium (from 12 to 9) levels of relative risk aversion the mean (over 1,000 replications) of the proportionate opportunity cost ranges from $0.3 \%(0.003)$ for the relative risk aversion of 12 to $1.0 \%$ (0.010) for relative risk aversion of 9 . This suggests that medium risk-tolerance investors also value optimal unconstrained portfolios but only to some extent, and require from $0.3 \%$ to $1.0 \%$ of additional initial wealth to stay constrained.

For high (from 29 to 31) levels of relative risk aversion the mean (over 1,000 replications) of the proportionate opportunity cost is $0.0 \%(0.000)$ in each case. This suggests that low risk-tolerance investors value optimal unconstrained portfolios as well as optimal constrained portfolios and do not require any additional payment to stay indifferent to being constrained. 
For ten assets, Table 2 shows that the lowest mean (over 1,000 replications) of the proportionate opportunity cost, $0.0 \%(0.000)$, corresponds to the high level of risk aversion of 31 . This means that an investor with the level of relative risk aversion of 31 being unconstrained will be equally happy as if he was constrained. The highest mean (over 1,000 replications) of the proportionate opportunity cost, $8.0 \%(0.080)$, corresponds to the very low level of relative risk aversion of 0.7 . This means that an investor with the level of relative risk aversion of 0.7 being unconstrained will be equally happy as if he was constrained but had $8.0 \%$ more of initial wealth.

The highest values of the proportionate opportunity cost (the means over 1,000 replications) correspond to low levels of relative risk aversion (from 0.7 to 3 ) and range from $2.1 \%(0.021)$ for the relative risk aversion of 3 to $8.0 \%(0.080)$ for the relative risk aversion of 0.7. Investors with low levels of risk aversion (from 3 to 0.7 ) in the presence of ten assets will require from $2.1 \%$ to $8.0 \%$ of initial wealth to stay constrained and being indifferent to the restrictions on short-selling. These magnitudes, as a matter of fact, are lower than those for the well-diversified number of assets in Table 1.

For medium (from 12 to 9) levels of relative risk aversion the mean (over 1,000 replications) of the proportionate opportunity cost is $0.6 \%(0.006)$ for risk aversion of 9 and 10 , and $0.5 \%(0.005)$ for risk aversion of 11 and 12 . These numbers are close to those for the well-diversified number of assets in Table 1. The explanation is that the welldiversified number of assets for medium levels of risk aversion is close to ten (see Table 3 and Table 4); therefore the proportionate opportunity cost in both cases will be about the same. 
For high (from 29 to 31) levels of relative risk aversion the mean (over 1,000 replications) of the proportionate opportunity cost is $0.1 \%$ (0.001) for risk aversion of 29 and 31 ; and $0.0 \%(0.000)$ for the risk aversion of 31 .

\subsubsection{Optimal Portfolio Shares}

Table 3 and Table 4 present typical optimal portfolio shares for unconstrained and constrained portfolio strategies for three different levels of relative risk aversion: low (of 0.7), medium (of 11) and high (of 31), for the well-diversified number of assets and for ten assets, in each case for a different set of available assets giving an opportunity cost that is typical for that level of risk aversion.

For both tables for unconstrained portfolios for risk aversion of 0.7 , more than $100 \%$ of initial wealth, $w_{0}$, is held in the nominally risky assets (asset \#1 through asset \#23 in Table 3 and asset \#1 through asset \#9 in Table 4) as a group, and Treasury bills are held in negative quantities.

As risk aversion increases, the proportion of initial wealth held in Treasury bills first, reaches zero, and then becomes positive; and correspondingly the proportion of initial wealth held in the group of nominally risky assets decreases.

The tables show that unconstrained optimal portfolio shares are not similar for different levels of risk aversion. As a matter of fact, optimal unconstrained portfolios for the low level of relative risk aversion of 0.7 have more extreme quantities (negative as well as positive) of assets than optimal unconstrained portfolios for medium level of relative risk aversion of 11 and for high level of relative risk aversion of 31. Extremely 


\section{Table 3}

Illustrative optimal portfolio shares for unconstrained portfolios and portfolios with the short-selling constraint for different values of relative risk aversion for the welldiversified number of assets ${ }^{1}$

\begin{tabular}{|c|c|c|c|c|c|c|}
\hline \multirow[t]{2}{*}{$\begin{array}{c}\text { \# of } \\
\text { An Asset }\end{array}$} & \multicolumn{2}{|c|}{$\begin{array}{l}\text { Relative Risk Aversion, }(1-\gamma), \\
\text { equal to } 0.7\end{array}$} & \multicolumn{2}{|c|}{$\begin{array}{c}\text { Relative Risk Aversion, } \\
(1-\gamma) \text {, equal to } 11\end{array}$} & \multicolumn{2}{|c|}{$\begin{array}{c}\text { Relative Risk Aversion, } \\
(1-\gamma) \text {, equal To } 31\end{array}$} \\
\hline & Unconstrained & Constrained & Unconstrained & Constrained & Unconstrained & Constrained \\
\hline 1 & 0.456 & 0.006 & 0.025 & 0.019 & 0.151 & 0.153 \\
\hline 2 & -0.103 & 0.003 & -0.103 & 0.025 & 0.007 & 0.007 \\
\hline 3 & -0.104 & -0.001 & 0.824 & -0.000 & - & - \\
\hline 4 & 1.634 & 0.082 & -1.777 & -0.446 & - & - \\
\hline 5 & 0.749 & 0.002 & 1.812 & 1.484 & - & - \\
\hline 6 & 0.219 & 0.004 & 0.589 & 0.026 & - & - \\
\hline 7 & 0.559 & -0.003 & - & - & - & - \\
\hline 8 & -0.678 & 0.001 & - & - & - & - \\
\hline 9 & 7.869 & 0.002 & - & - & - & - \\
\hline 10 & -0.985 & -0.002 & - & - & - & - \\
\hline 11 & -1.786 & +0.000 & - & - & - & - \\
\hline 12 & -1.614 & 0.004 & - & - & - & - \\
\hline 13 & -1.371 & 0.003 & - & - & - & - \\
\hline 14 & -1.574 & -0.002 & - & - & - & - \\
\hline 15 & 3.524 & 0.001 & - & - & - & - \\
\hline 16 & -0.389 & -0.001 & - & - & - & - \\
\hline 17 & 0.579 & 0.113 & - & - & - & - \\
\hline 18 & -0.861 & 0.189 & - & - & - & - \\
\hline 19 & 0.749 & 0.004 & - & - & - & - \\
\hline 20 & -0.466 & -0.004 & - & - & - & - \\
\hline 21 & 3.269 & 0.458 & - & - & - & - \\
\hline 22 & 2.426 & 0.793 & - & - & - & - \\
\hline 23 & -0.497 & 0.001 & - & - & - & - \\
\hline $24^{2}$ & -10.606 & -0.653 & -0.371 & -0.108 & 0.842 & 0.840 \\
\hline $\mathrm{E}\left(\mathrm{X}^{* \prime} \widetilde{R}\right)^{3}$ & 1.306 & 1.116 & 1.069 & 1.051 & 1.000 & 1.000 \\
\hline Certainty & & & & & & \\
\hline Equivalent & 1.187 & 1.052 & 1.044 & 1.040 & 0.999 & 0.999 \\
\hline
\end{tabular}

'Numbers are not comparable across levels of risk aversion, because for each level of risk aversion a different set of available assets was used: a set giving an exact value of opportunity cost typical for that level of risk aversion.

${ }^{2}$ The $24^{\text {th }}$ asset is risk-free in nominal terms.

${ }^{3}$ Monthly gross expected returns on portfolios. 
Table 4

Illustrative optimal portfolio shares for unconstrained portfolios and portfolios with the short-selling constraint for different values of relative risk aversion for ten $\operatorname{assets}^{1}$

\begin{tabular}{|c|c|c|c|c|c|c|}
\hline \multirow[t]{2}{*}{$\begin{array}{c}\text { \# of } \\
\text { An Asset }\end{array}$} & \multicolumn{2}{|c|}{$\begin{array}{l}\text { Relative Risk Aversion, }(1-\gamma) \text {, } \\
\text { equal to } 0.7\end{array}$} & \multicolumn{2}{|c|}{$\begin{array}{l}\text { Relative Risk Aversion, } \\
(1-\gamma) \text {, equal to } 11\end{array}$} & \multicolumn{2}{|c|}{$\begin{array}{c}\text { Relative Risk Aversion, } \\
(1-\gamma) \text {, equal to } 31\end{array}$} \\
\hline & Unconstrained & Constrained & Unconstrained & Constrained & Unconstrained & Constrained \\
\hline 1 & 0.707 & 0.626 & 0.132 & 0.109 & 0.010 & 0.010 \\
\hline 2 & 6.403 & +0.000 & 0.814 & 0.522 & -0.007 & -0.007 \\
\hline 3 & -2.541 & +0.000 & 0.263 & 0.225 & -0.004 & -0.004 \\
\hline 4 & -0.249 & -0.000 & -0.485 & -0.222 & -0.028 & -0.028 \\
\hline 5 & 2.174 & +0.000 & 0.130 & 0.106 & 0.011 & 0.011 \\
\hline 6 & -2.407 & -0.001 & 0.539 & 0.403 & 0.031 & 0.031 \\
\hline 7 & 2.050 & +0.000 & 0.231 & 0.117 & 0.008 & 0.008 \\
\hline 8 & -0.657 & -0.004 & 0.196 & 0.168 & 0.014 & 0.014 \\
\hline 9 & 3.648 & 1.368 & -0.267 & -0.128 & 0.118 & 0.118 \\
\hline $10^{2}$ & -8.129 & -0.999 & -0.553 & -0.301 & 0.846 & 0.846 \\
\hline $\mathrm{E}\left(\mathrm{X}^{* \prime} \widetilde{R}\right)^{3}$ & 1.243 & 1.102 & 1.047 & 1.034 & 1.002 & 1.002 \\
\hline $\begin{array}{c}\text { Certainty } \\
\text { Equivalent }\end{array}$ & 1.133 & 1.049 & 1.020 & 1.015 & 1.000 & 1.000 \\
\hline
\end{tabular}

${ }^{\prime}$ Numbers are not comparable across levels of risk aversion because for each level of risk aversion a different set of available assets was used: a set giving an exact value of opportunity cost typical for that level of risk aversion.

${ }^{2}$ The $10^{\text {th }}$ asset is risk-free in nominal terms.

${ }^{3}$ Monthly gross expected returns on portfolios.

negative quantities of assets for high risk-tolerance investors mean that the investors follow a very aggressive short-sale strategy.

The tables also show that constrained optimal portfolio shares are not similar for different levels of risk aversion as well. Optimal constrained portfolios for the low level of relative risk aversion of 0.7 have more extreme quantities (negative as well as positive) of assets than optimal constrained portfolios for medium level of relative risk aversion of 11 and for high level of relative risk aversion of 31. 
Also Table 3 and Table 4 show monthly expected returns on unconstrained and constrained optimal portfolios, $E\left(X^{* \prime} \widetilde{R}\right)$, for the three levels of relative risk aversion $(0.7,11$ and 31$)$.

The expected returns for constrained and unconstrained optimal portfolios for risk aversion of 0.7 are very dramatic for the well-diversified portfolios and large for ten-asset portfolios. Expected returns are of large size for risk aversion of 11 and of small size for risk aversion of 31. Such extreme magnitudes of expected portfolio returns for high risktolerance investors confirm the previously made conclusion about very aggressive shortsale strategies. These magnitudes represent very leveraged portfolios. For investors with risk aversion of 11 and 31 there is, definitely, some short-selling is going on too, but not as aggressive as for investors with risk aversion of 0.7 . The less aggressive short-selling for medium or high risk aversion leads to lower mean portfolio returns.

The big difference between the expected portfolio returns with the welldiversified number of assets and those with ten assets for the risk aversion of 0.7 is due to the level of diversification, which is optimal in the first case and sub-optimal in the second.

In comparing unconstrained expected portfolio returns and constrained expected portfolio returns for the three levels of risk aversion for the two tables I find that unconstrained and constrained expected portfolio returns for risk aversion of 31 are the same; for risk aversion of 11 they are close; for risk aversion of 0.7 unconstrained and constrained expected portfolio returns are not close at all. These unconstrained and constrained expected portfolio returns show that as risk aversion increases, the closer to each other expected returns on unconstrained and constrained portfolios are, and thus the 
more nearly indifferent an investor is between the unconstrained and constrained portfolio strategies. This happens due to the fact that as risk aversion increases, investors become less and less involved in short-selling; therefore, the short-selling constraint finally is not binding at all, and optimal unconstrained and constrained portfolio strategies more and more resemble each other.

Table 3 and Table 4 also report the certainty equivalents calculated for the same three levels of relative risk aversion $(0.7,11$ and 31$)$. The certainty equivalent, $(C E)$, is defined by

$$
\frac{1}{\gamma} C E^{\gamma}=\frac{1}{\gamma} w_{0}^{\gamma} E\left(\widetilde{R}^{\gamma}\right)
$$

and so, with $w_{0}=1$,

$$
C E=\left(E\left[\widetilde{R}^{\gamma}\right]\right)^{\frac{1}{\gamma}}
$$

The certainty equivalent represents the amount of certain wealth that would be viewed with indifference to the optimal portfolio. It is computed for investors of different levels of risk aversion: low (of 0.7), medium (of 11) and high (of 31). The two tables show that as risk aversion increases the value of the certainty equivalent decreases (for the unconstrained portfolio strategy as well as for the constrained). This suggests that as investors become more afraid of risk they use less risky portfolio strategies and will be expecting lower returns from those portfolios and, so, the certain amount of wealth they will be willing to accept with indifference will decrease.

It is interesting to compare certainty equivalents for unconstrained optimal portfolio strategies from Table 3 and Table 4 and the mean values of the proportionate opportunity cost from Table 1 and Table 2. For the well-diversified number of assets, as 
Table 1 shows, the mean over 1,000 replications of the proportionate opportunity cost reaches $12.8 \%$ for unconstrained investors with the level of risk aversion of 0.7 whose net certainty equivalent (the certainty equivalent minus 1.0 ) equals $18.7 \%$. As the level of risk aversion increases to 31 the mean of the proportionate opportunity cost falls to $0.0 \%$ while the net certainty equivalent falls to $-0.1 \%$.

For ten assets, as Table 2 shows, the mean value of the proportionate opportunity cost reaches $8.0 \%$ for unconstrained investors with their net certainty equivalent being equal to $13.3 \%$ for the risk aversion of 0.7 . As the level of risk aversion increases to 31 the mean of the proportionate opportunity cost for unconstrained investors falls to $0.0 \%$ while the net certainty equivalent falls to $0.0 \%$.

Table 5 presents the percentage of gross certainty equivalent for unconstrained portfolio strategies lost due to the short-selling constraint, computed as shown in (17). The percentage of gross certainty equivalent lost due to the constraint is timeless just like the proportionate opportunity cost, $\theta-1.0$.

$$
\text { The percentage loss }=\frac{C E^{\text {Uncd }}-C E^{\text {Const }}}{C E^{\text {Uncd }}}=\frac{\theta-1.0}{\theta}
$$

\section{Table 5}

The percentage of certainty equivalent lost due to the short-selling constraint for various levels of relative risk aversion with no exaggeration of extreme returns

\begin{tabular}{|c|c|c|c|c|c|c|c|c|c|c|c|}
\hline \multirow{2}{*}{$\begin{array}{c}\text { Number of } \\
\text { assets }\end{array}$} & \multicolumn{4}{|r|}{ Relative } & \multirow{2}{*}{$\begin{array}{c}\text { Risk } \\
9\end{array}$} & \multicolumn{6}{|c|}{ Aversion, $(1-\gamma)$} \\
\hline & 0.7 & 1 & 2 & 3 & & 10 & 11 & 12 & 29 & 30 & 31 \\
\hline $\begin{array}{c}\text { Well- } \\
\text { Diversified } \\
\text { Number }\end{array}$ & $11.3 \%$ & $7.9 \%$ & $6.4 \%$ & $3.8 \%$ & $1.0 \%$ & $0.8 \%$ & $0.4 \%$ & $0.3 \%$ & $0.0 \%$ & $0.0 \%$ & $0.0 \%$ \\
\hline 10 & $7.4 \%$ & $5.3 \%$ & $3.2 \%$ & $2.8 \%$ & $0.6 \%$ & $0.6 \%$ & $0.5 \%$ & $0.5 \%$ & $0.1 \%$ & $0.1 \%$ & $0.0 \%$ \\
\hline
\end{tabular}

The percentage loss $=\frac{C E^{\text {Uncd }}-C E^{\text {Cond }}}{C E^{\text {Uncd }}} * 100 \%=\frac{\theta-1}{\theta} * 100 \%$. 
The highest percentage loss, $11.3 \%$, happens for the investors with risk aversion of 0.7 holding the well-diversified number of assets in their portfolios. As risk aversion increases, for both well-diversified and ten-asset portfolios, the percentage loss decreases. The lowest percentage loss, $0.0 \%$, is observed for the investors with risk aversion of 31 holding ten assets in their portfolios and for investors with the well-diversified optimal portfolios of risk aversion of 29 and higher. Table 5 confirms the previously made conclusion that as investors become more afraid of risk their perceptions of the optimal constrained and optimal unconstrained portfolio strategies become more and more similar. Hence, as the level of risk aversion increases the certain amount of wealth unconstrained investors and constrained investors will be willing to accept with indifference will be getting closer to each other, and, therefore, the percentage loss in certainty equivalents due to the constraint will decrease.

Table 3 and Table 4 show that magnitudes of portfolio shares for different levels of relative risk aversion, as well as for unconstrained and constrained portfolio strategies at low risk aversion, are very different. Comparison of optimal portfolio shares across different levels of risk aversion is meaningless, since portfolio shares for different levels of relative risk aversion were calculated by using different sets of available assets for each level of risk aversion. But comparison of unconstrained and constrained optimal portfolios is very interesting and possible (constrained and unconstrained portfolio shares for a particular level of relative risk aversion correspond to the same set of available assets). 
In terms of comparing unconstrained and constrained optimal portfolios, I find in Table 3 and Table 4 that absolute values of shares of unconstrained portfolios are, in almost all cases, bigger than those of constrained portfolios.

Table 6 and Table 7 present correlation coefficients and geometric distances calculated between unconstrained and constrained portfolio share vectors for different levels of relative risk aversion: low, (from 0.7 to 3), medium (from 9 to 12) and high (from 29 to 31), for all assets in ones' portfolio (the well-diversified number and ten) and separately for the group of nominally risky assets only (asset \#1 through asset \#23 in Table 6 and asset \#1 through asset \#9 in Table 7).

Both tables clearly show an increase in correlation between unconstrained and constrained optimal portfolio shares as risk aversion increases. But correlation coefficients calculated for all assets in portfolios (the well-diversified number or ten) are higher that those calculated for the nominally risky assets only.

The increase in correlation coefficients can be explained by the presence of Treasury bills in the well-diversified optimal portfolios and ten-asset optimal portfolios. For the low levels of risk aversion when investors use highly leveraged portfolios they go very short on Treasury bills in unconstrained strategies and do not place any portion of their initial wealth into Treasury bills under the constrained strategy. This difference in fund allocations produces relatively small correlation. But as risk aversion increases, the investor will place a bigger and bigger portion of their initial wealth into Treasury bills, and will reduce their participation in short-selling. These two factors will push unconstrained and constrained portfolio strategies toward each other. Hence, we see an 
Table 6

Illustrative correlation coefficients and geometric distances for unconstrained portfolios and portfolios with the short-selling constraint for different levels of relative risk aversion for the well-diversified number of assets ${ }^{1}$

\begin{tabular}{|c|c|c|c|c|}
\hline $\begin{array}{c}\text { Relative } \\
\text { Risk } \\
\text { Aversion, }(1-\gamma)\end{array}$ & $\begin{array}{c}\text { Correlation } \\
\text { Coefficients } \\
\text { for all assets }\end{array}$ & $\begin{array}{c}\text { Geometric } \\
\text { Distance } \\
\text { for all assets }\end{array}$ & $\begin{array}{c}\text { Correlation Coefficients for } \\
\text { the group of nominally risky } \\
\text { assets only }\end{array}$ & $\begin{array}{c}\text { Geometric Distance }{ }^{2} \text { for } \\
\text { nominally risky assets only }\end{array}$ \\
\hline 0.7 & 0.549 & 14.136 & 0.311 & 10.038 \\
1 & 0.591 & 8.326 & 0.473 & 6.631 \\
2 & 0.613 & 4.413 & 0.560 & 4.115 \\
3 & 0.609 & 3.047 & 0.591 & 2.937 \\
9 & 0.793 & 1.991 & 0.715 & 1.861 \\
10 & 0.814 & 1.819 & 0.789 & 1.763 \\
11 & 0.823 & 1.721 & 0.817 & 1.640 \\
12 & 0.861 & 1.409 & 0.843 & 1.307 \\
29 & 1.000 & 0.002 & 1.000 & 0.001 \\
30 & 1.000 & 0.002 & 1.000 & 0.001 \\
31 & 1.000 & 0.002 & 1.000 & 0.001 \\
\hline
\end{tabular}

${ }^{\prime}$ Calculated for different fixed sets of assets for different levels of risk aversion; in each case the asset set is the one giving an opportunity cost typical for that level of risk aversion

${ }^{2}$ Geometric distance is defined as $\left[(X-\hat{X})^{\prime}(X-\hat{X})\right]^{1 / 2}$ where $X$ is the unconstrained portfolio, and $\hat{X}$ is the constrained portfolio.

Table 7

Illustrative correlation coefficients and geometric distances for unconstrained portfolios and portfolios with the short-selling constraint for different levels of relative risk aversion for ten assets ${ }^{1}$

\begin{tabular}{|c|c|c|c|c|}
\hline $\begin{array}{c}\text { Relative } \\
\text { Risk } \\
\text { Aversion, }(1-\gamma)\end{array}$ & $\begin{array}{c}\text { Correlation } \\
\text { Coefficients } \\
\text { for 10 assets }\end{array}$ & $\begin{array}{c}\text { Geometric } \\
\text { Distance } \\
\text { for 10 assets }\end{array}$ & $\begin{array}{c}\text { Correlation Coefficients for } \\
\text { the group of nominally risky } \\
\text { Assets only (first 9 assets) }\end{array}$ & $\begin{array}{c}\text { Geometric Distance }{ }^{2} \text { for } \\
\text { nominally risky assets only } \\
\text { (first } 9 \text { assets) }\end{array}$ \\
\hline 0.7 & 0.634 & 10.895 & 0.309 & 8.238 \\
1 & 0.663 & 6.309 & 0.415 & 5.831 \\
2 & 0.649 & 4.136 & 0.409 & 4.097 \\
3 & 0.701 & 2.013 & 0.593 & 1.895 \\
9 & 0.893 & 1.029 & 0.872 & 0.093 \\
10 & 0.956 & 0.713 & 0.921 & 0.457 \\
11 & 0.984 & 0.522 & 0.980 & 0.409 \\
12 & 0.992 & 0.412 & 0.991 & 0.001 \\
39 & 1.000 & 0.001 & 1.000 & 0.001 \\
31 & 1.000 & 0.001 & 1.000 & 0.001 \\
\hline
\end{tabular}

${ }^{1}$ Calculated for different fixed sets of assets for different levels of risk aversion; in each case the asset set is the one giving an opportunity cost typical for that level of risk aversion.

${ }^{2}$ Geometric distance is defined as $\left[(X-\hat{X})^{\prime}(X-\hat{X})\right]^{1 / 2}$ where $X$ is the unconstrained portfolio, and $\hat{X}$ is the constrained portfolio. 
increase in the correlation coefficients. For the levels of risk aversion of 29 and higher there is a perfect correlation between unconstrained and constrained portfolio shares. That means that unconstrained and constrained portfolio strategies at those levels of risk aversion are virtually the same.

The geometric distance calculated for different values of risk aversion is another way to compare unconstrained and constrained portfolios. Geometric distance is defined as $\left[(X-\hat{X})^{\prime}(X-\hat{X})\right]^{1 / 2}$ where $X$ is the unconstrained portfolio, and $\hat{X}$ is the constrained portfolio. The greater the geometric distance between the two portfolios is, the further the unconstrained optimal portfolio is from the constrained optimal portfolio, the greater an investor's welfare loss is likely to be if he must choose the constrained portfolio, and so the higher the opportunity cost for the investor is. Table 6 and Table 7 show that as risk aversion increases the geometric distance between unconstrained and constrained portfolios decreases, making unconstrained and constrained portfolios more similar to each other. So, if the unconstrained optimal portfolio and the constrained optimal portfolio are getting closer as risk aversion increases, I would anticipate that the opportunity cost will decrease. That is exactly my finding from Table 1 and Table 2.

\subsubsection{Regret in the Worst-Case Scenario}

Large negative and positive asset holdings (Table 3 and Table 4) in unconstrained portfolios and to some extent in constrained portfolios for investors with a level of risk aversion of 0.7 suggest that the investors take on a lot of risk. This raises the question: if the worst possible portfolio outcome occurs, then how much will the investors suffer 
from such an outcome? It is possible to measure the investors' proportionate regret from the worst-case scenario with such a risky portfolio.

Table 8 and Table 9 report the proportionate regret, $(\theta-1)$, for the welldiversified number of assets and for ten assets, that will be incurred by investors if the worst possible outcome of asset returns occurs. This $\theta$ is defined by

$$
U\left(\theta\left(X^{* \prime} R\right)^{\text {worst }}\right)=E U\left(X^{* \prime} \widetilde{R}\right)
$$

where $X^{*}$ is the optimally chosen portfolio, $\left(X^{* \prime} R\right)^{\text {worst }}$ is the one of the 120 states of nature giving the lowest portfolio return, $U\left[\left(X^{* \prime} R\right)^{\text {worst }}\right]$ is an investor's utility from getting the worst possible portfolio outcome, $E U\left(X^{* \prime} \widetilde{R}\right)$ is an investor's ex ante expected utility.

For unconstrained investors (for the case with the well-diversified number of assets as well as for ten assets) the mean of the proportionate regret (over 1,000 replications) is the highest for the low level of risk aversion of 0.7 and the lowest for the high level of risk aversion of 31 . This means that high risk-tolerance investors do choose very risky unconstrained asset allocations. And it is riskier when the number of assets is at the well-diversified level. Those asset allocations are so risky at the level of risk aversion of 0.7 , that if the worst possible outcome occurs it would require for investors with the well-diversified number of assets to receive $1021.0 \%$ of initial wealth in compensation and for investors with ten assets to receive $641.2 \%$ of initial wealth in order to get the same level of ex post utility as their ex ante expected utility. For the high level of 31 for risk aversion the mean of the proportionate regret (over 1,000 replications) is $2.0 \%(0.020)$ for investors with well-diversified number of assets and $2.8 \%(0.028)$ for 


\section{Table 8}

The ex post proportionate regret, $(\theta-1)$, under the worst portfolio outcome for the well-diversified number of assets

\begin{tabular}{|c|c|c|c|c|c|c|c|}
\hline $\begin{array}{c}\text { Relative Risk } \\
\text { Aversion, (1- } \gamma \text { ) }\end{array}$ & Portfolios & $\begin{array}{c}\text { \# of } \\
\text { Assets }\end{array}$ & Smallest & Mean & Median & Largest & $\begin{array}{c}\text { Standard } \\
\text { Deviation }\end{array}$ \\
\hline \multirow{2}{*}{$\mathbf{0 . 7}$} & Unconstrained & 24 & 2.628 & 10.210 & 9.244 & 31.444 & 4.168 \\
& Constrained & 24 & 0.309 & 1.109 & 0.902 & 4.331 & 0.486 \\
\hline \multirow{2}{*}{$\mathbf{1 1}$} & Unconstrained & 7 & 0.029 & 0.077 & 0.069 & 0.614 & 0.047 \\
& Constrained & 7 & 0.029 & 0.076 & 0.069 & 0.386 & 0.037 \\
\hline & Unconstrained & 3 & 0.004 & 0.020 & 0.015 & 0.163 & 0.017 \\
& Constrained & 3 & 0.004 & 0.020 & 0.015 & 0.127 & 0.016 \\
\hline
\end{tabular}

Table 9

The ex post proportionate regret, $(\theta-1)$, under the worst portfolio outcome for ten assets

\begin{tabular}{|c|c|c|c|c|c|c|}
\hline $\begin{array}{c}\text { Relative Risk } \\
\text { Aversion, (1- } \gamma \text { ) }\end{array}$ & Portfolios & Smallest & Mean & Median & Largest & Standard Deviation \\
\hline \multirow{2}{*}{$\mathbf{0 . 7}$} & Unconstrained & 0.345 & 6.412 & 5.015 & 18.159 & 2.696 \\
& Constrained & 0.227 & 1.009 & 0.849 & 2.742 & 1.269 \\
\hline \multirow{2}{*}{$\mathbf{1 1}$} & Unconstrained & 0.034 & 0.083 & 0.079 & 0.608 & 0.035 \\
& Constrained & 0.034 & 0.082 & 0.079 & 0.327 & 0.026 \\
\hline & Unconstrained & 0.014 & 0.028 & 0.027 & 0.098 & 0.009 \\
& Constrained & 0.014 & 0.028 & 0.027 & 0.094 & 0.009 \\
\hline
\end{tabular}


investors with ten assets. Such a low proportionate regret suggests that low risk-tolerance unconstrained investors choose very conservative unconstrained asset allocations. So conservative are their allocations that even the worst possible outcome will require for them less than $3.0 \%$ of initial wealth to get to the same level of utility as their ex ante expected utility.

For constrained portfolio strategies the mean proportionate regret (over 1,000 replications) ranges from $110.9 \%$ (1.109) for risk aversion of 0.7 to $2.0 \%(0.020)$ for risk aversion of 31 for investors with the well-diversified number of assets, and from $100.9 \%$ (1.009) for risk aversion of 0.7 to $2.8 \%$ (0.028) for risk aversion of 31 for investors with ten assets. This means that constrained portfolios have a very restrictive character and do not let high risk-tolerance investors take nearly as much risk as they would in the absence of the constraint. For low risk-tolerance investors constrained portfolios are virtually the same as unconstrained portfolios (the difference in the mean regret is $0.0 \%$ ) and represent very conservative asset allocations with very little risk to take. Note that the tendency for the short-selling constraint to make portfolios more conservative is also seen in Table 3 and Table 4, which show that at each level of risk aversion the mean portfolio return is less when the constraint is present than when it is not.

\subsection{Historical Data with Extreme Values Exaggerated}

In order to check the robustness of the estimates of the proportionate opportunity cost I include extremely high and extremely low simulated asset returns in each data set of available assets (well-diversified portfolios as well as ten-asset portfolios). 
I construct the simulated extremely high and extremely low asset returns the following way. For the original data set for each historical time period I compute the average excess return across all assets in the data set. A historical time period with the highest average excess return across all assets defines the historical period with the highest returns. A historical time period with the lowest average excess return across all assets defines the historical period with the lowest returns. Then, for the extreme historical periods only I calculate the deviation of each asset's return from that asset's intertemporal mean return. The deviations are doubled and then added back to assets' intertemporal means. This way I create two fictional time periods with exaggerated high and exaggerated low returns. These fictional asset returns provide simulated extreme time periods to replace the time periods they were constructed from. The rest of the original data set remains unchanged.

Then I repeat the whole procedure of calculating the proportionate opportunity cost 1,000 times in each case simulating two fictional data periods. This gives me 1,000 new $\theta$ 's from the 1,000 data sets with extreme returns exaggerated. The results from this project are reported in Table 10 for the well-diversified number of assets and Table 11 for ten assets.

Out of all values considered for relative risk aversion, the lowest mean (over 1,000 replications) of the proportionate opportunity cost for the well-diversified number of assets and for ten assets corresponds to the high level of relative risk aversion of 31 . The highest mean (over 1,000 replications) of the proportionate opportunity cost for the well-diversified number of assets and for ten assets corresponds to the low level of relative risk aversion of 0.7 . 
Table 10

The proportionate opportunity cost of the short-selling constraint, $(\theta-1)$, for various values of relative risk aversion with extreme returns exaggerated for the well-diversified number of assets

\begin{tabular}{|c|c|c|c|c|c|c|}
\hline $\begin{array}{c}\text { Relative } \\
\text { Risk Aversion, (1- } \gamma \text { ) }\end{array}$ & $\begin{array}{c}\text { \# of } \\
\text { Assets }\end{array}$ & Smallest & Mean & Median & Largest & $\begin{array}{c}\text { Standard } \\
\text { Deviation }\end{array}$ \\
\hline Low & & & & & & \\
$\mathbf{0 . 7}$ & 25 & 0.047 & 0.135 & 0.129 & 0.586 & 0.061 \\
$\mathbf{1}$ & 24 & 0.031 & 0.091 & 0.089 & 0.570 & 0.049 \\
$\mathbf{2}$ & 23 & 0.010 & 0.073 & 0.068 & 0.487 & 0.025 \\
$\mathbf{3}$ & 21 & 0.005 & 0.061 & 0.053 & 0.439 & 0.012 \\
Medium & & & & & & \\
$\mathbf{9}$ & 11 & 0.001 & 0.016 & 0.000 & 0.199 & 0.005 \\
$\mathbf{1 0}$ & 10 & 0.001 & 0.010 & 0.000 & 0.181 & 0.005 \\
$\mathbf{1 1}$ & 9 & 0.000 & 0.009 & 0.000 & 0.163 & 0.004 \\
$\mathbf{1 2}$ & 8 & 0.000 & 0.006 & 0.000 & 0.150 & 0.003 \\
High & & & & & & \\
$\mathbf{2 9}$ & 4 & 0.000 & 0.001 & 0.000 & 0.004 & 0.001 \\
$\mathbf{3 0}$ & 3 & 0.000 & 0.001 & 0.000 & 0.003 & 0.001 \\
$\mathbf{3 1}$ & 3 & 0.000 & 0.000 & 0.000 & 0.003 & 0.001 \\
\hline
\end{tabular}

Table 11

The proportionate opportunity cost of the short-selling constraint, $(\theta-1)$, for various values of relative risk aversion with extreme returns exaggerated for ten assets

\begin{tabular}{|c|c|c|c|c|c|}
\hline $\begin{array}{c}\text { Relative } \\
\text { Risk Aversion, (1- } \gamma)\end{array}$ & Smallest & Mean & Median & Largest & Standard Deviation \\
\hline \multicolumn{6}{|l|}{ Low } \\
\hline 0.7 & 0.029 & 0.091 & 0.121 & 0.516 & 0.051 \\
\hline 1 & 0.016 & 0.069 & 0.080 & 0.509 & 0.039 \\
\hline 2 & 0.006 & 0.047 & 0.061 & 0.453 & 0.022 \\
\hline 3 & 0.003 & 0.033 & 0.031 & 0.419 & 0.010 \\
\hline \multicolumn{6}{|l|}{ Medium } \\
\hline 9 & 0.001 & 0.014 & 0.000 & 0.160 & 0.006 \\
\hline 10 & 0.001 & 0.009 & 0.000 & 0.154 & 0.005 \\
\hline 11 & 0.000 & 0.007 & 0.000 & 0.143 & 0.005 \\
\hline 12 & 0.000 & 0.005 & 0.000 & 0.131 & 0.004 \\
\hline \multicolumn{6}{|l|}{ High } \\
\hline 29 & 0.000 & 0.001 & 0.000 & 0.004 & 0.001 \\
\hline 30 & 0.000 & 0.001 & 0.000 & 0.003 & 0.001 \\
\hline 31 & 0.000 & 0.001 & 0.000 & 0.003 & 0.001 \\
\hline
\end{tabular}


The results in the second project confirm the results from the first one: as the level of relative risk aversion increases the proportionate opportunity cost decreases, the better the constrained portfolio performs ex ante and the lower the proportion of initial wealth an investor requires to stay constrained and accept the short-selling restriction. Also, the same way as with the original data set, as the level of relative risk aversion increases the standard deviation of the proportionate opportunity cost decreases. This means that as the level of risk aversion increases, as investors facing different asset sets become less risk tolerant, their perceptions of the optimal constrained portfolio strategy, even with extreme historical periods, are more similar to each other than perceptions of the strategy for investors with lower risk aversion.

The only difference between Tables 1-2 and Tables $10-11$ is that the magnitude of the proportionate opportunity cost is bigger when extreme returns exaggerated. These differences can be explained by the following. The calculation of the proportionate opportunity cost in Tables 10 and 11 was based on the historical asset returns distribution with the extreme returns exaggerated. This exaggeration of historically occurred extreme returns converted the original asset returns distribution, derived as shown in (4)-(9), into a distribution with fatter tails. This change in the distribution increases the probability for investors to end up with extremely low portfolio returns. Hence, the proportionate opportunity cost of using the constrained optimal portfolio instead of the unconstrained one in the case of exaggeration of extreme returns will be higher.

The lowest mean (over 1,000 replications) of the proportionate opportunity cost for the well-diversified number of assets (Table 10) is $0.0 \%(0.000)$ and corresponds to the level of risk aversion of 31 . This means that an investor with the level of relative risk 
aversion of 31 being unconstrained will be equally happy as if he was constrained. The highest mean (over 1,000 replications) of the proportionate opportunity cost, $13.5 \%$ (0.135), corresponds to the very low level of relative risk aversion of 0.7 . This means that an investor with the level of relative risk aversion of 0.7 being unconstrained will be equally happy as if he was constrained but had $13.5 \%$ more of initial wealth.

The lowest mean (over 1,000 replications) of the proportionate opportunity cost for ten assets (Table 11) is $0.1 \%(0.001)$ and corresponds to the level of risk aversion of 29 and higher. The highest mean (over 1,000 replications) of the proportionate opportunity cost, $9.1 \%(0.091)$, corresponds to the very low level of relative risk aversion of 0.7 .

Table 12 presents the percentage of gross certainty equivalent for unconstrained portfolio strategies lost due to the short-selling constraint, computed as shown in (17), with extreme returns exaggerated in the asset return distribution. The percentage of gross certainty equivalent lost due to the constraint is timeless just like the proportionate opportunity cost, $\theta-1.0$.

Table 12

The percentage of certainty equivalent lost due to the short-selling constraint for various levels of relative risk aversion with extreme returns exaggerated

\begin{tabular}{|c|c|c|c|c|c|c|c|c|c|c|c|}
\hline \multirow{2}{*}{$\begin{array}{c}\text { Number of } \\
\text { assets }\end{array}$} & \multicolumn{5}{|c|}{ Relative Risk } & \multicolumn{6}{|c|}{ Aversion, $(1-\gamma)$} \\
\hline & 0.7 & 1 & 2 & 3 & 9 & 10 & 11 & 12 & 29 & 30 & 31 \\
\hline $\begin{array}{c}\text { Well- } \\
\text { Diversified } \\
\text { Number }\end{array}$ & $11.9 \%$ & $8.3 \%$ & $6.8 \%$ & $5.7 \%$ & $1.6 \%$ & $1.0 \%$ & $0.9 \%$ & $0.6 \%$ & $0.1 \%$ & $0.1 \%$ & $0.0 \%$ \\
\hline 10 & $8.3 \%$ & $6.5 \%$ & $4.5 \%$ & $3.2 \%$ & $1.4 \%$ & $0.9 \%$ & $0.7 \%$ & $0.5 \%$ & $0.1 \%$ & $0.1 \%$ & $0.1 \%$ \\
\hline
\end{tabular}

The percentage loss $=\frac{C E^{\text {Uncd }}-C E^{\text {Cond }}}{C E^{\text {Uncd }}} * 100 \%=\frac{\theta-1}{\theta} * 100 \%$. 
The highest percentage loss, $11.9 \%$, happens for the investors with risk aversion of 0.7 holding the well-diversified number of assets in their portfolios. As risk aversion increases, for both well-diversified portfolios and ten-asset portfolios, the percentage loss decreases. The lowest percentage loss, $0.0 \%$, is observed for the investors with the welldiversified number of assets for risk aversion of 31 . Table 12 confirms the results from Table 5, and the previously made conclusion that as investors become more afraid of risk their perceptions of the optimal constrained and optimal unconstrained portfolio strategies become more and more similar. Hence, as the level of risk aversion increases the certain amount of wealth unconstrained investors as well as constrained investors will be willing to accept with indifference will be getting closer to each other, and, therefore, the percentage loss in certainty equivalents due to the constraint will decrease.

The only difference between Table 5 and Table 12 is that the magnitude of the percentage loss is bigger when extreme returns are exaggerated. This is another confirmation of the previously made conclusion that when extreme returns are exaggerated in the historical distribution it will produce fatter tails in the distribution. So, the cost of choosing the constrained portfolio this time will be higher and fatter tails will increase the size of the certainty equivalent loss from choosing the constrained portfolio strategy instead of the optimal portfolio strategy.

\section{Conclusion}

In this essay I have investigated the opportunity cost incurred by investors when they use optimal portfolios constrained by a short-selling restriction instead of unconstrained optimal portfolios. Two sets of returns have been used: the original 
historical asset returns and historical asset returns with extreme values exaggerated. CRRA utility functions and the proportionate opportunity cost have been used. The opportunity cost has been calculated for different values of relative risk aversion (including extreme levels of relative risk aversion) for the well-diversified portfolios and ten-asset portfolios. The highest mean across simulations of the proportionate opportunity cost found is $13.5 \%(0.135)$ for the level of relative risk aversion of 0.7 with extreme returns exaggerated for the well-diversified optimal portfolios. The lowest mean of the proportionate opportunity cost found is $0.0 \%(0.000)$ for the level of relative risk aversion of 29 and higher for the original historical asset returns for the well-diversified optimal portfolios and for risk aversion of 31 for the ten-asset portfolio. For both data sets, for the original historical asset returns and for historical asset returns with extreme values exaggerated, as the level of relative risk aversion increases the proportionate opportunity cost decreases.

The only difference between estimates of the proportionate opportunity cost for these two data sets is the magnitude of the estimates. They are bigger for the data sets when extreme returns exaggerated. This can be explained by the fact that the presence of extreme returns in the probability distribution creates fatter tails in the distribution which increases the opportunity cost of accepting the constraint.

Therefore, based on my calculations, I may conclude that for investors with low levels of risk aversion the short-selling constraint represents a serious hazard. For investors with medium levels of relative risk aversion the constrained portfolio strategy performs quite well. For investors with very high levels of relative risk aversion (29 and 
above) the optimal portfolio strategy with the short-selling constraint performs as well as the unconstrained portfolio strategy. 


\section{References:}

Best, Michael J., Grauer, Rober R., On the Sensitivity of Mean-Variance-Efficient Portfolios to Changes in Asset Means: Some Analytical and Computational Results, Review of Financial Studies, 1991, 4, 315-342

Campbell, John Y., Viceira, Luis H., Strategic Asset Allocation, Oxford University Press, March, 2002

Luttmer Erzo G. J., Asset Pricing in Economies with Frictions, Econometrica, 1996, 64, $1439-1467$

Tepla, Lucie, Optimal Portfolio Policies with Borrowing and Shortsale Constraints, Journal of Economic Dynamics and Control, 2000, 24, 1623-1639

Tew, B.V., Reid, D.W., Witt, C.A., The Opportunity Cost of a Mean-Variance Efficient Choice, The Financial Review, 1991, 26(1), 31-43

Wang, Zhenyu, Efficiency Loss and Constraints on Portfolio Holdings, Journal of Financial Economics, 1998, 48, 359-375 


\section{Chapter 5: Conclusions and Prospects for Future Research}

This dissertation has explored the effect of different constrained portfolio strategies on investors' welfare. A constrained portfolio strategy gives investors a suboptimal asset allocation that results in investors' welfare losses. To measure those welfare losses I compared optimal constrained portfolios with optimal unconstrained portfolios by using the concept of the proportionate opportunity cost along with various constant relative risk aversion utility functions. Two sets of asset returns were used in calculations: the original historical asset returns data set, and the historical asset returns data set with extreme values exaggerated. In each data set case, with a nominally risk-free asset, a vector autoregression was used to generate the joint distribution of asset returns for the portfolio formation period, and 1,000 alternative asset sets were randomly made available for investment.

The introductory chapter discussed reasons the investors use constrained portfolio strategies, described the different constrained portfolio strategies that were used in the dissertation, derived the proportionate opportunity cost, described the derivation of the joint probability distribution function for asset returns, and described the way the historical extreme asset return values were exaggerated to check the robustness of the estimates of the proportionate opportunity cost.

The first essay analyzed investors' welfare losses from being constrained to choose a mean-variance efficient portfolio strategy. I showed that the mean-variance 
strategy shows a moderately good approximation to the optimal unconstrained portfolio strategy. When extreme values of asset returns were not exaggerated in the asset returns distribution, investors' welfare losses did not exceed $5.6 \%$ of the initial wealth. With extreme values of asset returns exaggerated in the asset returns distribution, investors' welfare losses did not exceed $11 \%$ of the initial wealth. The results in both cases indicated that as the number of assets in investors' portfolios increased investors welfare losses from the mean-variance constraint increased as well, and that less risk-averse investors experienced greater welfare losses.

The second essay analyzed investors' welfare losses from investing in a suboptimal number of assets. The analysis was performed with and without a short-selling constraint. I showed that without the short-selling constraint and with no exaggeration of extreme values of asset returns the well-diversified number of assets for an investor with low risk aversion was 24 . The number decreased as the level of risk aversion increased, and when the short-selling constraint was introduced. When the extreme values of asset returns were exaggerated in the asset returns distribution the well-diversified number of assets increased.

The second part of the second essay explored investors' welfare losses when they restricted themselves to invest in either stocks or bonds but not both. To measure those welfare losses I compared "only stock indices and Treasury bills" optimal portfolios or "only bond indices and Treasury bills" optimal portfolios with "stock and bond indices and Treasury bills" optimal portfolios. I showed that for investors with low levels of risk aversion welfare losses did not exceed $1.5 \%$ of initial wealth when they invested sub- 
optimally. For investors with medium and high levels of relative risk aversion, constrained portfolios that included only one type of assets, stocks only or bonds only, along with Treasury bills, gave expected utility about as high as unconstrained portfolios that included both types of assets, stocks and bonds.

One of the future research ideas with respect to the optimal number of assets in one's portfolio is to answer the question: how well-diversified are balanced publicly available mutual funds? If one buys a share in a balanced mutual fund, will he be investing optimally? In other words, if publicly available balanced mutual funds are not well-diversified, then by buying a share in just one mutual fund the investor will create a sub-optimal portfolio and that will result in a welfare loss for the investor.

The third essay analyzed investors' welfare losses from being restricting from short-selling. I showed that with a nominally risk-free asset the optimal portfolio strategy with the short-selling constraint performed almost as well as the unconstrained portfolio strategy for investors with medium levels of risk aversion, and performed as well as the unconstrained portfolio strategy for investors with high levels of risk aversion. The results, derived from the original historical asset returns data set with no exaggeration of extreme returns, showed that investors' welfare losses reached $13.7 \%$ of initial wealth when risk aversion was low. With extreme returns exaggerated in the returns distribution, investors' welfare losses reached $14.2 \%$ of initial wealth. The results in both cases indicated that less risk-averse investors experienced greater welfare losses, and that the short-selling constraint reduced the cost of sub-optimal diversification. 
Another example of a constrained portfolio strategy for a future research idea is so-called "naïve" portfolios. "Naïve" portfolios are portfolios in which $1 / n$ of initial wealth of an investor is invested in every one of $n$ assets. This "naïve" portfolio strategy does not involve any optimization. Therefore, "naïve" portfolios are sub-optimal and will cause investors welfare losses.

Another future research idea concerns international diversification. Optimal portfolios that include only domestic assets are constrained portfolios in terms of types of assets involved. A globally well-diversified portfolio includes not only domestic assets but also international assets. If an investor decides to invest in domestic assets only, constraining himself that way to only one type of assets, he creates a constrained optimal portfolio, which will result in a sub-optimal asset allocation. A sub-optimal asset allocation will cause investors welfare losses.

Other ideas that may be considered for future research come from different types of assets that are not included in investors' portfolios. Investors will incur welfare losses from not including in their portfolios such assets as real estate, precious metals, smalland large-capital stocks, and so forth. 


\section{Appendix 1:}

\section{Derivation of Theta}

A constant relative risk aversion (CRRA) utility function:

$$
\begin{aligned}
& U(\widetilde{w})=\frac{1}{\gamma} w^{\gamma}, \text { where } \gamma \neq 0 \text { and } \gamma<1 . \\
& E U\left(\theta w_{0} \widetilde{R}^{\text {constrained }}\right)=E U\left(w_{0} \widetilde{R}^{\text {unconstrained }}\right)
\end{aligned}
$$

With CRRA above I have:

$$
\begin{gathered}
E\left(\frac{1}{\gamma}\left(\theta w_{0} \widetilde{R}^{\text {constrainead }}\right)^{\gamma}\right)=E\left(\frac{1}{\gamma}\left(w_{0} \widetilde{R}^{\text {unconstrained }}\right)^{\gamma}\right) \\
\frac{1}{\gamma} \theta^{\gamma} w_{0}^{\gamma} E\left(\widetilde{R}^{\text {constrained }}\right)^{\gamma}=\frac{1}{\gamma} w_{0}^{\gamma} E\left(\widetilde{R}^{\text {unconstrained }}\right)^{\gamma} \\
\theta^{\gamma} E\left(\widetilde{R}^{\text {constrained }}\right)^{\gamma}=E\left(\widetilde{R}^{\text {unconstrained }}\right)^{\gamma} \\
\theta^{\gamma}=\frac{E\left(\widetilde{R}^{\text {unconstrained }}\right)^{\gamma}}{E\left(\widetilde{R}^{\text {constrained }}\right)^{\gamma}} \\
\theta=\left[\frac{E\left(\widetilde{R}^{\text {unconstrained }}\right)^{\gamma}}{E\left(\widetilde{R}^{\text {constrained }}\right)^{\gamma}}\right] \frac{1}{\gamma}
\end{gathered}
$$




\section{Appendix 2:}

Sample SAS Program for Calculating the Opportunity Cost of the MeanVariance Constraint.

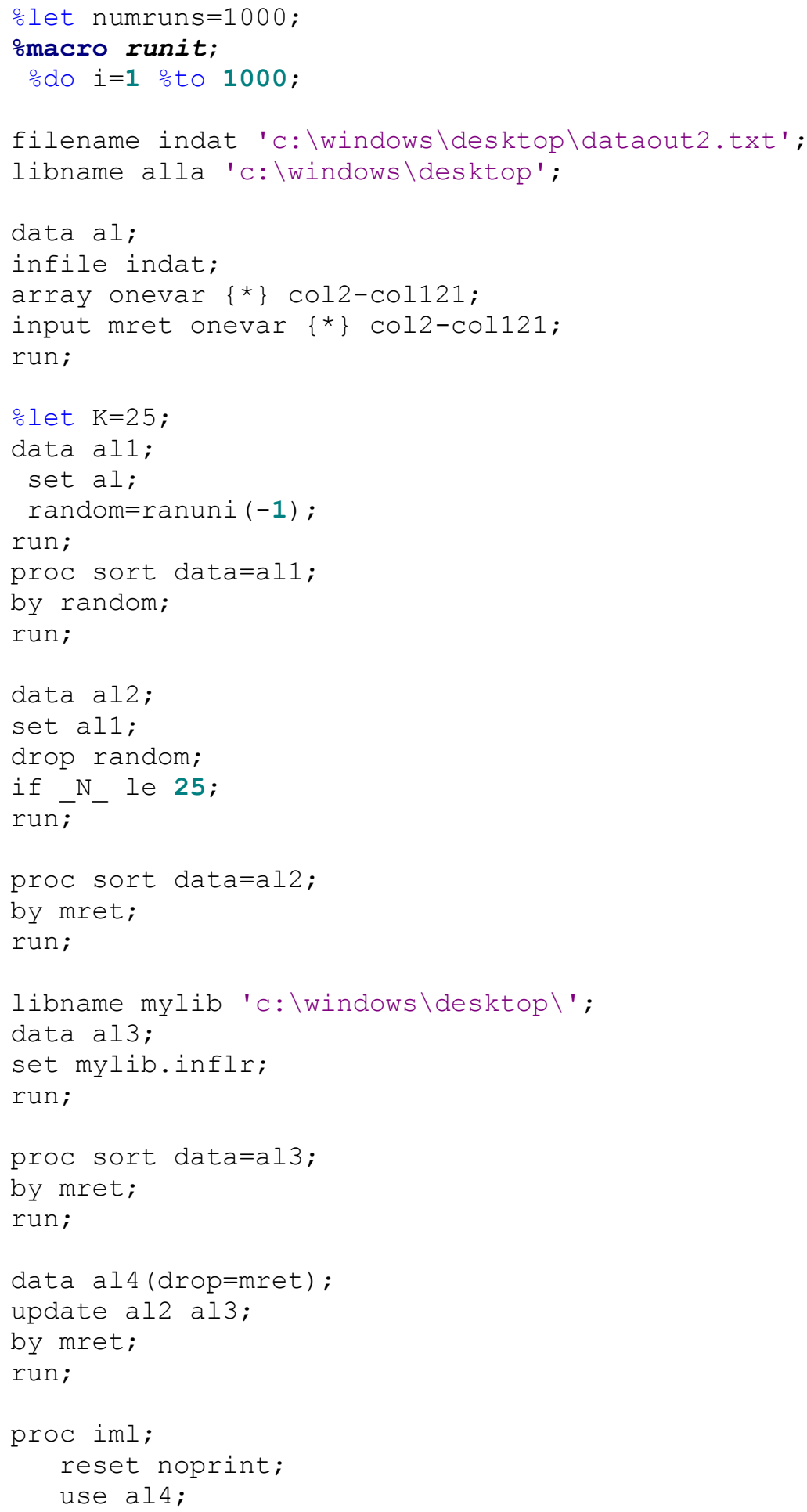




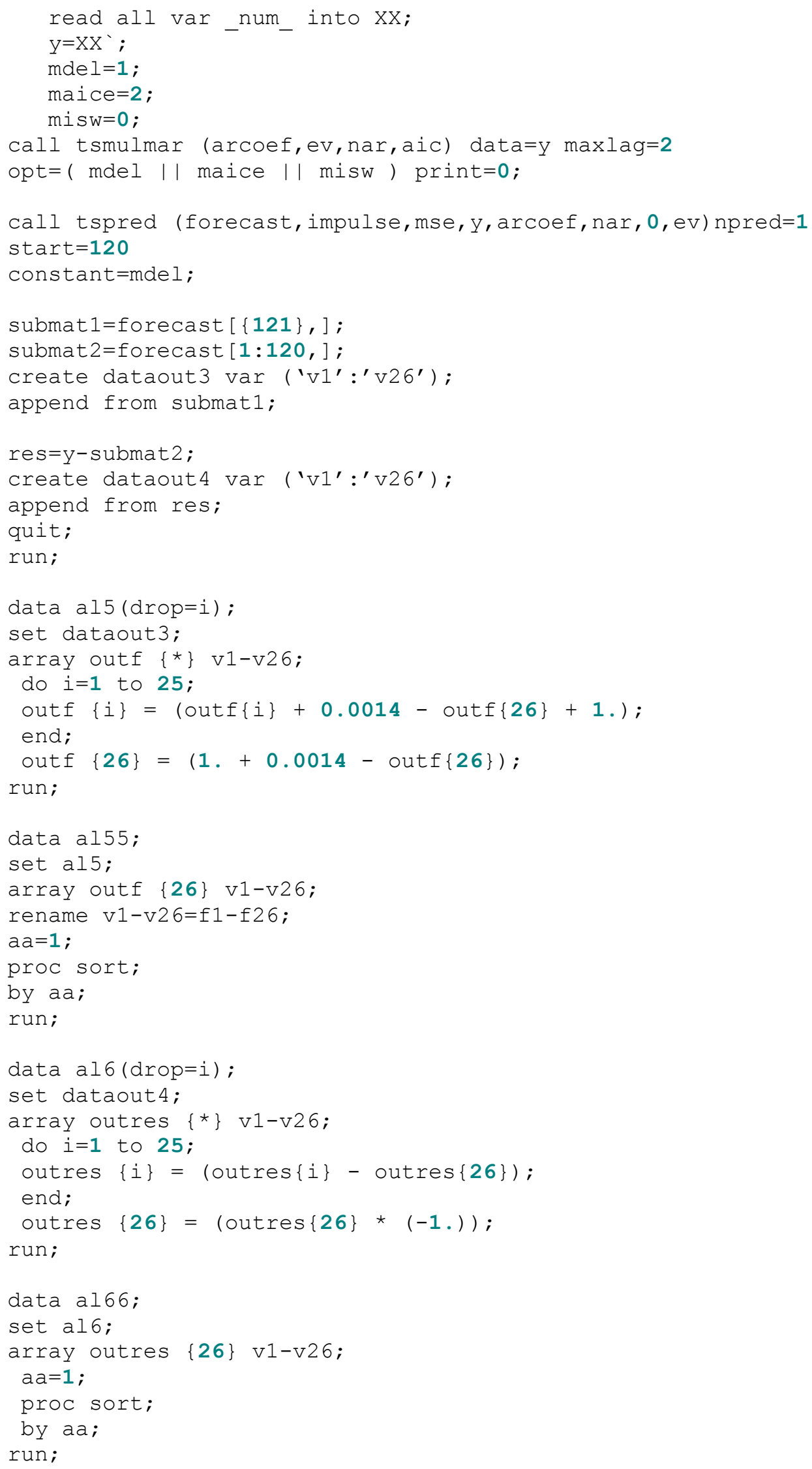




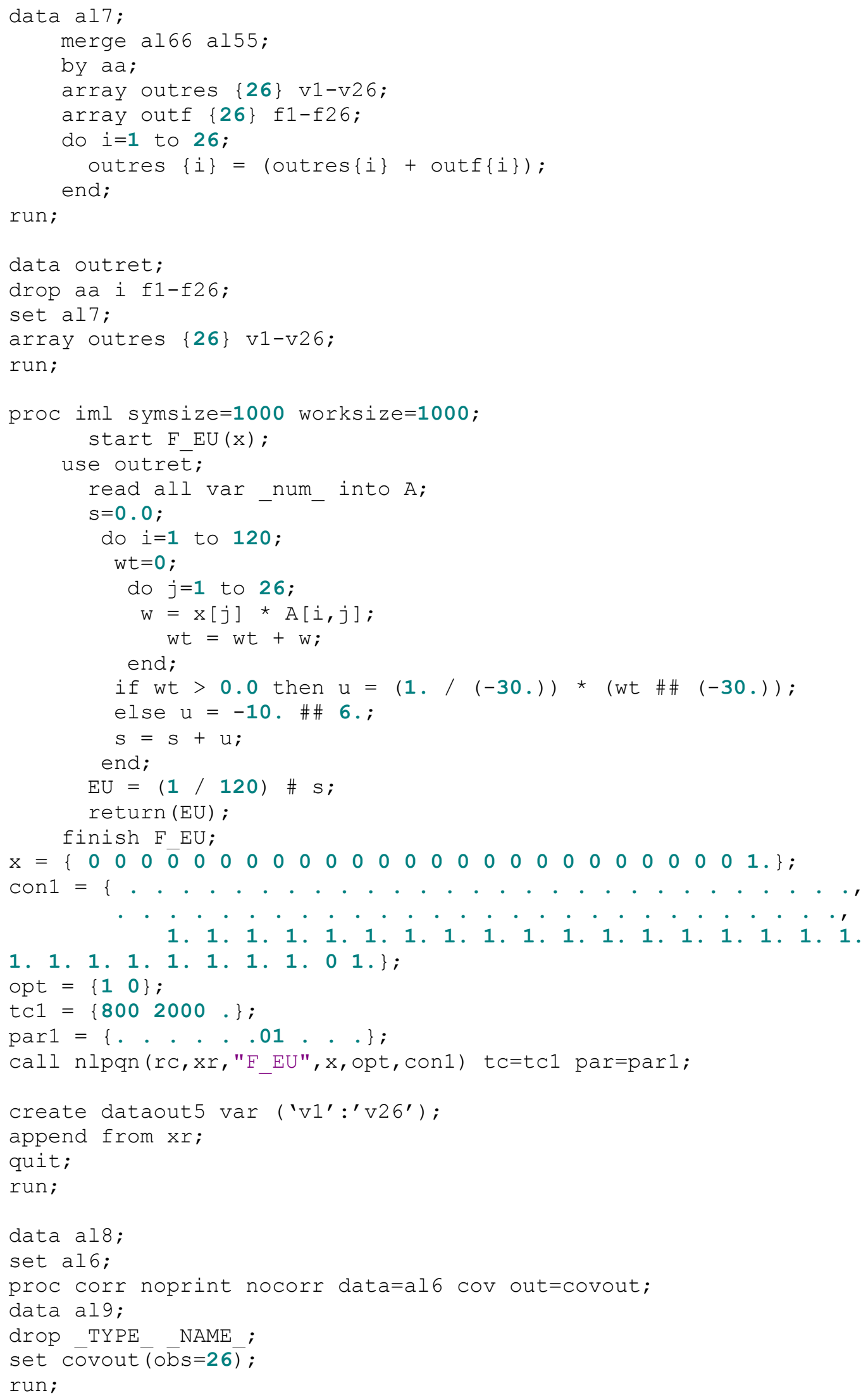




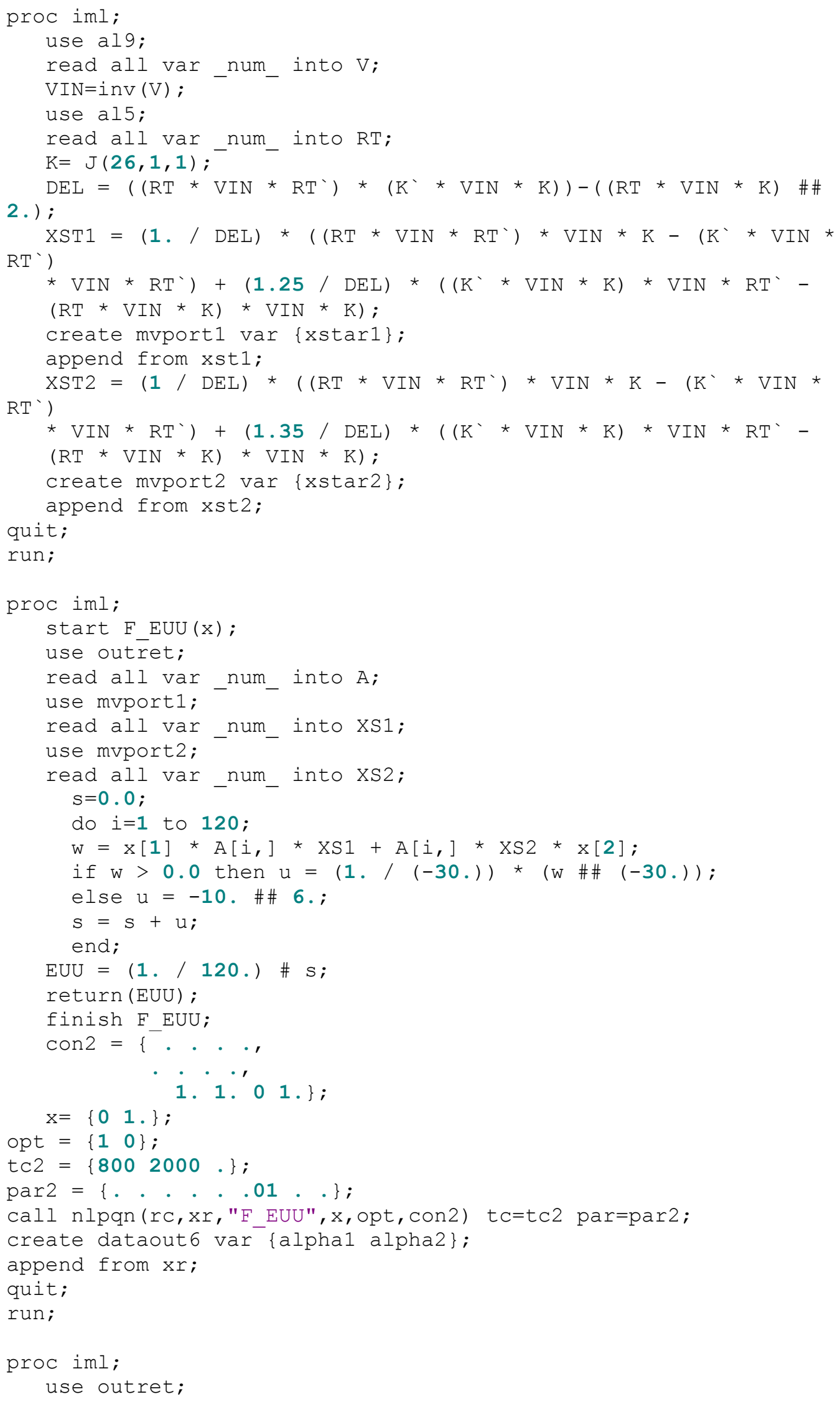




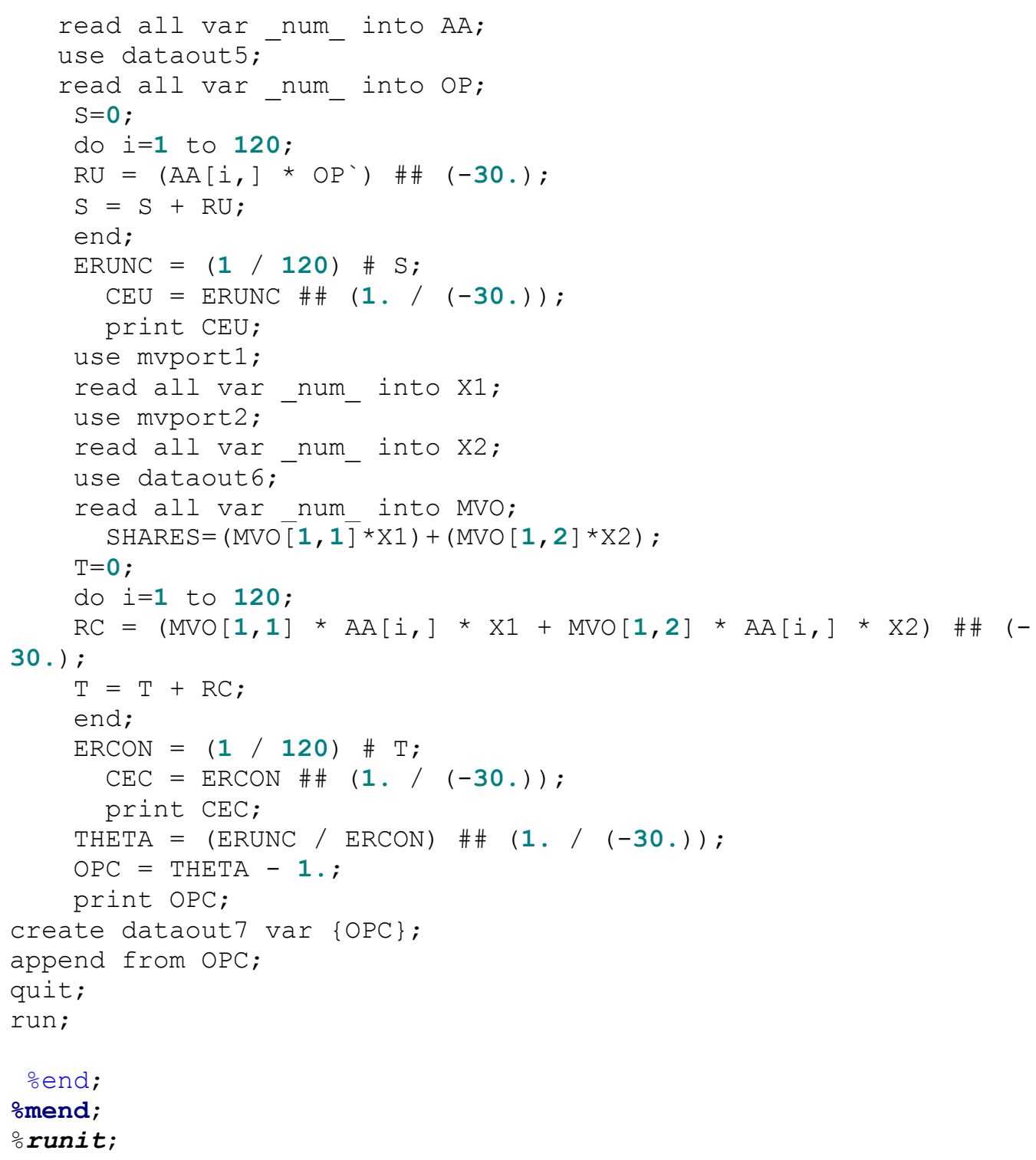

